\title{
AN AFFINE ALMOST POSITIVE ROOTS MODEL
}

\author{
NATHAN READING AND SALVATORE STELLA
}

\begin{abstract}
We generalize the almost positive roots model for cluster algebras from finite type to a uniform finite/affine type model. We define a subset $\Phi_{c}$ of the root system and a compatibility degree on $\Phi_{c}$, given by a formula that is new even in finite type. The clusters (maximal pairwise compatible sets of roots) define a complete fan $\operatorname{Fan}_{c}(\Phi)$. Equivalently, every vector has a unique cluster expansion. We give a piecewise linear isomorphism from the subfan of $\operatorname{Fan}_{c}(\Phi)$ induced by real roots to the g-vector fan of the associated cluster algebra. We show that $\Phi_{c}$ is the set of denominator vectors of the associated acyclic cluster algebra and conjecture that the compatibility degree also describes denominator vectors for non-acyclic initial seeds. We extend results on exchangeability of roots to the affine case.
\end{abstract}

\section{CONTENTS}

1. Introduction 1

2. Background 5

3. The roots $\Phi_{c} \quad 12$

4. The compatibility degree $\quad 15$

5. Compatibility and clusters 20

6. Cluster expansions and the cluster fan 24

7. Exchangeable roots 29

8. Type-by-type arguments for exchangeability 35

9. Connections with Cluster algebras: g-Vectors and d-vectors 39

Index to notation $\quad 43$

References $\quad 44$

\section{INTRODUCTION}

One of the first major achievements in the structural theory of cluster algebras was the classification, by Fomin and Zelevinsky [17, 18], of cluster algebras of finite type (cluster algebras with finitely many seeds). The classification parallels the Cartan-Killing classification, and an acyclic cluster algebra of finite type is specified by a Cartan matrix of finite type, an orientation of its Dynkin diagram (which together specify an exchange matrix) and a choice of "coefficients." The cluster variables in the cluster algebra are in bijection with the almost positive roots (roots that either are positive or are the negatives of simples) in the corresponding root system. The bijection sends a cluster variable to its denominator vector (or d-vector), leading to a purely combinatorial model based on almost positive roots,

Nathan Reading was supported in part by NSF grants DMS-1101568 and DMS-1500949.

Salvatore Stella was partially supported by NCSU and INdAM and ISF grant 1144/16. 
generalizing the associahedron or Stasheff polytope. The almost positive roots also model [42] the g-vectors of cluster variables.

After Cartan matrices of finite type, the next simplest Cartan matrices are those of affine type. An affine Cartan matrix together with an acyclic orientation of its Dynkin diagram and a choice of coefficients specifies a cluster algebra of affine type. Except in rank 2, these are precisely the cluster algebras whose "growth rate" is linear, in the sense of [15]. (Every $2 \times 2$ Cartan matrix gives rise to a cluster algebra that is finite or has linear growth.) Another intrinsic characterization of affine type is a consequence of [40, Theorem 3.5]: An $n \times n$ exchange matrix with $n \geq 3$ is of finite or affine type if and only if it is of finite mutation type and is mutation equivalent to an acyclic exchange matrix.

In this paper, we extend the almost positive roots model to cluster algebras of affine type. In particular, we extend many of the main results in $[11,17,18]$ to affine type. We define an affine generalized associahedron fan, extending the normal fan of generalized associahedra from finite type to a uniform finite/affine type construction. We do not, at this time, have an affine analog of the generalized associahedra as polytopes.

From a certain point of view, one might say that the almost positive roots model has already been extended to affine type and beyond in the representation theory literature. In that setting, the denominator vectors are dimension vectors of indecomposable rigid representation and the compatibility degree is the dimension of $\mathrm{Ext}^{1}$ between the representations $[3,5,7]$. There is also an approach to denominator vectors in the surfaces model [16, Section 6]. However, neither of these successful models is an almost positive roots model. We provide such a model by first defining explicitly a set of roots $\Phi_{c}$ in terms of the action of a Coxeter element on the root system, and then defining a compatibility degree in terms of multiplication and addition of simple-root (or coroot) coordinates or elementary counting with supports. In the representation theoretic and surface models, by contrast, the definitions are in terms of modules and $\mathrm{Ext}^{1}$ or in terms of tagged arcs and intersection numbers. Our model agrees with the surfaces model but only agrees with the representation theory model up to the notion of compatibility. Details on the relationship between our model and these other models are found in remarks at the end of this introduction. We now give details on our model and results.

In affine type, taking $\tau_{c}$ to be the usual deformation of $c$, the set $\Phi_{c}$ is the union of the $\tau_{c}$-orbits of the positive roots that do not have full support, together with the imaginary root $\delta$. (See Definition 3.1 and Proposition 3.13 for several other characterizations of $\Phi_{c}$.)

We give a new formula for the usual finite-type compatibility degree $(\alpha \| \beta)_{c}$ $[18,26]$ and extend the formula to affine type. Two roots in $\Phi_{c}$ are $c$-compatible if their compatibility degree is zero. We give the affine versions of the key properties already known in finite type, including cardinalities of clusters (maximal sets of pairwise $c$-compatible roots in $\Phi_{c}$ ). We also prove the existence of unique cluster expansions (Theorem 6.2), or in other words, we show (Theorem 6.4) that the nonnegative spans of clusters are the maximal cones of a complete fan $\operatorname{Fan}_{c}(\Phi)$. Finally, we give the affine version of the characterization of exchangeability in terms of compatibility degree.

We now describe the connection to cluster algebras. Suppose $B$ is an exchange matrix arising from a Cartan matrix $A$ of affine type and an acyclic orientation of 
the associated Dynkin diagram. The orientation of the Dynkin diagram is encoded in $B$ as a choice of signs of off-diagonal entries. We write $\mathcal{A}_{\bullet}(B)$ for the principalcoefficients cluster algebra determined by $B$. Let $c$ be the Coxeter element obtained by multiplying the simple reflections $S$ in an order such that $s_{i}$ precedes $s_{j}$ if $b_{i j}>0$. The notation $\operatorname{Fan}_{c}^{\mathrm{re}}(\Phi)$ denotes the subfan of $\operatorname{Fan}_{c}(\Phi)$ consisting of cones spanned by clusters not containing $\delta$, and $\nu_{c}$ is a piecewise linear map defined in Section 9 .

Theorem 1.1. Suppose that $B$ is an acyclic exchange matrix whose associated Cartan matrix is of affine type. Let $\Phi$ be the associated root system and $c$ the associated Coxeter element.

(1) The piecewise-linear map $\nu_{c}$ induces an isomorphism from $\operatorname{Fan}_{c}^{\mathrm{re}}(\Phi)$ to the $\mathbf{g}$ vector fan of $\mathcal{A}_{\mathbf{0}}(B)$.

(2) The cluster complex of $\mathcal{A}_{\bullet}(B)$ is isomorphic to the simplicial complex underlying $\operatorname{Fan}_{c}^{\mathrm{re}}(\Phi)$.

The finite-type version of the theorem follows immediately from [29, Theorem 8.1] and [34, Theorem 5.39]. We prove Theorem 1.1 using results (proved here and in $[34,35])$ that use only the combinatorics of root systems and Coxeter groups. In contrast, the following result requires a theorem ([38, Proposition 9], quoted here as Theorem 9.4) that is proved using representation theory. Given a seed $\Sigma$ (essentially a choice of $B$ and coefficients), write $\mathcal{A}_{\Sigma}$ for the cluster algebra determined by $\Sigma$.

Theorem 1.2. Suppose $\Sigma$ is a seed with exchange matrix $B$ that is acyclic and whose associated Cartan matrix is of affine type. Let $\Phi$ be the associated root system and $c$ the associated Coxeter element. The map from cluster variables to $\mathbf{d}$-vectors is a bijection to $\Phi_{c} \backslash\{\delta\}$. The nonnegative linear spans of $\mathbf{d}$-vectors of clusters in $\mathcal{A}_{\Sigma}$ are the maximal cones of a fan, which coincides with $\operatorname{Fan}_{c}^{\mathrm{re}}(\Phi)$.

Ceballos and Pilaud [10, Corollary 3.3] showed that the almost positive roots model describes denominator vectors in all finite types (without requiring acyclicity). We conjecture that the same is true in affine type. We write $\mathbf{d}(x)$ or $\mathbf{d}_{\Sigma}(x)$ for the denominator vector of a cluster variable $x$ with respect to an initial seed $\Sigma$.

Conjecture 1.3. Suppose $\Sigma$ is an acyclic seed of affine type with exchange matrix $B$, associated root system $\Phi$ and associated Coxeter element $c$. Index the cluster variables of $\mathcal{A}_{\Sigma}$ as $x(\beta)$ for $\beta \in \Phi_{c}$ according to the bijection in Theorem 1.2. Given any seed $\Sigma^{\prime}$ mutation-equivalent to $\Sigma$ and with the cluster in $\Sigma^{\prime}$ indexed as $x\left(\beta_{1}\right), \ldots, x\left(\beta_{n}\right)$ and given any $\beta \in \Phi_{c}$, we have

$$
\mathbf{d}_{\Sigma^{\prime}}(x(\beta))=\left(\left(\beta_{1} \| \beta\right)_{c}, \ldots,\left(\beta_{n} \| \beta\right)_{c}\right) .
$$

After playing a crucial role in the classification of cluster algebras of finite type (as described above), $\mathbf{d}$-vectors have fallen somewhat out of favor, because $\mathbf{g}$-vectors appear to have nicer properties. However, [10, Corollary 3.3] and Conjecture 1.3 exhibit a nice property of $\mathbf{d}$-vectors that appears to have no analog for $\mathbf{g}$-vectors. In Section 9, we discuss evidence for Conjecture 1.3, including the case of surfaces, many cases where $\Sigma^{\prime}$ is also acyclic, and some computational evidence.

Remark 1.4 (Applications). The connections to cluster algebras mentioned above concern $\operatorname{Fan}_{c}^{\mathrm{re}}(\Phi)$, which is only part of the complete fan $\operatorname{Fan}_{c}(\Phi)$, but we expect the entire fan $\operatorname{Fan}_{c}(\Phi)$ to be important to the theory of cluster algebras. As part of work in progress, we have constructed cluster scattering diagrams (in the sense 
of [20]) of affine type, and proved that the fan defined by these cluster scattering diagrams coincides with $\nu_{c} \operatorname{Fan}_{c}(\Phi)$. Furthermore, we have proved that the mutation fan (in the sense of [30]) coincides with $\nu_{c} \operatorname{Fan}_{c}(\Phi)$. As a consequence, we expect to prove a conjectured description of affine universal geometric cluster algebras [30, Conjecture 10.15]. We also intend to study, with Jon McCammond, the surprising similarities between $\operatorname{Fan}_{c}(\Phi)$ and a lattice constructed by McCammond and Sulway [27] to prove longstanding conjectures about Euclidean Artin groups. (Compare especially [27, Table 1] and [27, Proposition 7.6] with Table 2.1 and Proposition 5.13.)

We now make some remarks on the connection between the current paper and the existing literature. In particular, Remarks 1.6, 1.8 and 1.9 describe the relationship between our results and some related representation-theoretic results.

Remark 1.5 (Related work on the affine case). Theorems 1.1 and 1.2 build affine $\mathbf{g}$-vector fans and $\mathbf{d}$-vector fans in terms of compatibility of roots in $\Phi_{c}$. Affine $\mathbf{g}$ vector fans were previously constructed in [35] as doubled Cambrian fans. In particular, cones were constructed by specifying their normal vectors. The construction here gives g-vectors directly (rather than by inverting a matrix) and provides a direct test for compatibility (whether two cluster variables are in a common cluster) and exchangeability (whether they are in adjacent clusters). Affine d-vectors were also constructed in [4], as we will discuss further in Remark 1.8. Affine cluster algebras of rank 2 are treated in $[8,28,41,44]$. The affine types (except for a finite number of exceptional cases) can be approached through the surfaces model [16, Section 6] or the orbifolds model [15]. Finally, [37] characterizes cluster variables of affine type as generalized minors.

Remark 1.6 (Schur roots). Caldero and Keller [7, Theorem 4] showed, for any acyclic quiver, that the cluster character map [6] is a bijection from the set of indecomposable objects without self-extensions in the cluster category to the set of cluster variables. They also showed [7, Theorem 3] that the dimension vector of the object is the $\mathbf{d}$-vector of the corresponding cluster variable. The dimension vectors of these indecomposable objects are the real Schur roots. Thus the first assertion in our Theorem 1.2 combines with the Caldero-Keller results to identify the roots $\Phi_{c}$ as follows:

Corollary 1.7. If $B$ is acyclic and skew-symmetric, with associated root system $\Phi$ and Coxeter element $c$, then the positive real roots in $\Phi_{c}$ are precisely the Schur roots for the corresponding quiver.

Our Theorem 1.2 uses Theorem 9.4, which is proved in [38] using the same circle of ideas that appear in [7] (generalized to the skew-symmetrizable setting). It seems likely that one could use ideas from $[7,38]$ to prove Corollary 1.7 directly, and then cite [7, Theorem 3] (or its generalization [38, Proposition 5]) to prove Theorem 1.2. In any case, in light of Corollary 1.7, it seems reasonable to call $\Phi_{c}$ the "almost positive Schur roots". Comparison with $[22,39]$ makes it clear that $\Lambda_{c}=\Phi_{c}^{\mathrm{re}} \cap U^{c}$ (Definition 3.1) is the set of dimension vectors of the regular representations. As usual, the deformed Coxeter element $\tau_{c}$ corresponds to the Auslander-Reiten translation (or its inverse, depending on conventions).

Remark 1.8 (Compatibility degree, Ext $^{1}$, and Hom). One representation-theoretic notion of compatibility degree is the dimension of Ext ${ }^{1}$ between the corresponding 
modules in the cluster category. Comparing [7, Theorems 3 and 4] to our Theorems 1.1 and 1.2 and comparing [39, Lemma 3.2] or [22, Lemma 5.3] with our Proposition 5.6, we see that our compatibility degree on distinct roots is zero if and only if the $\operatorname{Ext}^{1}$ compatibility degree is zero. In other words, our notion of $c$-compatibility (Definition 5.1) agrees with the $\operatorname{Ext}^{1}$ notion of compatibility. However, beyond that, the two notions of compatibility degree need not agree. By $[3$, Theorem 7.5], the $\operatorname{Ext}^{1}$ compatibility degree of real roots $\alpha$ and $\beta$ is 1 in both directions if and only if $\alpha$ and $\beta$ are real $c$-exchangeable in the sense of Definition 7.1. Theorem 7.2 shows that our $c$-compatibility degree on real roots is 1 in both directions if and only if the two roots are $c$-exchangeable (as opposed to real $c$-exchangeable). The notions of $c$-exchangeability and real $c$-exchangeability are distinct, as made clear in Theorem 7.2.

In $[4$, Theorem A], denominator vectors in affine type are described in terms of the dimensions of certain spaces of homomorphisms. (This is not called a "compatibility degree" in [4], but perhaps should be.) If Conjecture 1.3 is true, then our $c$-compatibility degree constitutes a root-theoretic formulas for these dimensions.

Remark 1.9 (Cluster expansions and canonical decompositions). Since compatibility is the same in our setting and the representation-theoretic setting, our cluster expansions (Definition 6.1) are analogous to the canonical decompositions (or generic decompositions) of [23]. Thus, parts of Proposition 5.14 correspond to results proved in [39, Section 3] and [22, Section 6]. The canonical decomposition fan constructed in [22] coincides with $\operatorname{Fan}_{c}(\Phi)$ within the span of the positive roots.

We conclude this introduction with a remark about some potentially simplifying choices that we did not make.

Remark 1.10. In constructing the $\mathbf{d}$-vector fan and $\mathbf{g}$-vector fan, we could have saved some complications by ignoring the imaginary root $\delta$. However, the complete fan that we obtain by including $\delta$ is crucial to the future work mentioned in Remark 1.4. Similarly, if our goals were entirely combinatorial, we could have restricted our attention to the standard affine root systems (the affinizations of finite root systems). However, the full range of affine root systems is needed in order to model all cluster algebras of affine type. Because we didn't make these simplifications, some results cannot be stated uniformly. Rather, there are exceptions in one affine type: Type $A_{2 k}^{(2)}$ in Kac's notational system [24, §4.8]. This is the unique type with three distinct root lengths (except when $k=1$, when there are two lengths with ratio 2).

\section{BACKGROUND}

2.1. Root systems and Coxeter groups. We assume familiarity with the most basic theory of Cartan matrices, root systems and Coxeter groups, and in this background section the goal is to establish terminology and notation and to recall some some of the less basic aspects of the theory.

Let $n$ be a positive integer. A Cartan matrix is a symmetrizable integer matrix $A=\left[a_{i j}\right]_{1 \leq i, j \leq n}$, with symmetrizing constants $d_{i}$ (so that $d_{i} a_{i j}=d_{j} a_{j i}$ for all $i, j$ ).

Let $V$ be a real vector space with basis $\Pi=\left\{\alpha_{1}, \ldots, \alpha_{n}\right\}$. The $\alpha_{i}$ are the simple roots. The simple co-roots are $\alpha_{i}^{\vee}=d_{i}^{-1} \alpha_{i}$ and $\Pi^{\vee}$ is the set of simple co-roots. Given $v \in V$ and $\alpha_{i} \in \Pi$, we write $\left[v: \alpha_{i}\right.$ ] for the coefficient of $\alpha_{i}$ when $v$ is expanded in the basis of simple roots. Let $V^{*}$ be the dual space to $V$ and let $\langle\cdot, \cdot\rangle$ be the 
canonical pairing between $V^{*}$ and $V$. The fundamental weights $\left\{\rho_{i}: i=1, \ldots, n\right\}$ are the basis of $V^{*}$ that is dual to the basis $\Pi^{\vee}$ of co-roots.

The Cartan matrix $A$ encodes a symmetric bilinear form $K$ on $V$ defined by $K\left(\alpha_{i}^{\vee}, \alpha_{j}\right)=a_{i j}$. For each $i=1, \ldots, n$, the simple reflection $s_{i}$ is defined on the basis $\Pi$ by $s_{i}\left(\alpha_{j}\right)=\alpha_{j}-K\left(\alpha_{i}^{\vee}, \alpha_{j}\right) \alpha_{i}$. Equivalently, $s_{i}\left(\alpha_{j}^{\vee}\right)=\alpha_{j}^{\vee}-K\left(\alpha_{j}^{\vee}, \alpha_{i}\right) \alpha_{i}^{\vee}$. The Weyl group $W$ is the group generated by $S=\left\{s_{i}: i=1, \ldots, n\right\}$. Each $s_{i}$ acts as a reflection with respect to $K$, and thus the action of $W$ preserves $K$. As usual, the action of $W$ on $V^{*}$, dual to its action on $V$, is given by $\langle w \phi, v\rangle=\left\langle\phi, w^{-1} v\right\rangle$.

Remark 2.1. Our convention places both roots and co-roots in the space $V$ and places weights and co-weights in the dual space $V^{*}$. The standard Lie-theoretic setup places roots and weights in $V^{*}$ and co-roots and co-weights in $V$. The approach used here matches the approach implied in [2, Chapter 4] and the approach in $[34,32,33,35]$. Furthermore, while the scattering diagram construction in [20] is not phrased in terms of roots and weights, it is naturally rephrased in those terms, and this rephrasing also follows the convention of the present paper.

The real roots are the vectors $w \alpha_{i} \in V$ for $w \in W$ and $i=1, \ldots, n$, and the real co-roots are the vectors $w \alpha_{i}^{\vee}$. The real root system $\Phi^{\mathrm{re}}$ is the set of all real roots. The root system is a larger set $\Phi$, containing $\Phi^{\text {re }}$ (strictly when $\Phi^{\text {re }}$ is infinite). The set $\Phi \backslash \Phi^{\text {re }}$ is the set of imaginary roots. We do not need the full generality of imaginary roots, so we do not define them here.

The root system $\Phi$ is a subset of the root lattice (the lattice spanned by $\Pi$ ), and is the disjoint union of positive roots $\Phi^{+}=\left\{\beta \in \Phi:\left[\beta: \alpha_{i}\right] \geq 0\right.$ for $\left.i=1, \ldots n\right\}$ and negative roots $\Phi^{-}=-\Phi^{+}$. For each real root $\beta$ there is a co-root $\beta^{\vee}=\frac{2}{K(\beta, \beta)} \beta$. There is a bijection $\beta \mapsto t_{\beta}$ between real positive roots and reflections in $W$ given by $t_{\beta}(v)=v-K\left(\beta^{\vee}, v\right) \beta$ for $v \in V$.

A Coxeter element $c$ is the product of any permutation of $S$. Mostly, we fix $c$ and assume that $\Pi$ has been indexed so that $c=s_{1} \cdots s_{n}$. But sometimes we let $c$ vary, usually without referring directly to any numbering of the simple reflections. For $s \in S$, we say $s$ is initial in $c$ if $c$ has a reduced word whose first letter is $s$ and $s$ is final in $c$ if $c$ has a reduced word whose last letter is $s$. A source-sink move is the operation of replacing $c$ by the Coxeter element scs for $s$ initial or final in $c$.

Given a reduced word $s_{1} \cdots s_{n}$ for $c$, the Euler form $E_{c}$ is defined on the bases of simple roots and co-roots by

$$
E_{c}\left(\alpha_{i}^{\vee}, \alpha_{j}\right)=\left\{\begin{array}{ll}
a_{i j} & \text { if } i>j, \\
1 & \text { if } i=j, \\
0 & \text { if } i<j .
\end{array}\right. \text { or }
$$

Since $a_{i j}=K\left(\alpha_{i}^{\vee}, \alpha_{j}\right)$, for any $\alpha, \beta \in V$ we have

$$
K(\alpha, \beta)=E_{c}(\alpha, \beta)+E_{c}(\beta, \alpha) .
$$

The form $E_{c}$ depends on $c$ but is independent of the choice of reduced word for $c$. Some facts about it will be useful; the first is [33, Lemma 3.3].

Lemma 2.2. If $s$ is initial or final in $c$, then $E_{c}(\alpha, \beta)=E_{s c s}(s \alpha, s \beta)$ for all $\alpha$ and $\beta$ in $V$.

Applying Lemma 2.2 repeatedly with $s$ running backwards through a reduced word for $c$, we obtain the following fact: 
Lemma 2.3. $E_{c}(\alpha, \beta)=E_{c}(c \alpha, c \beta)$ for all $\alpha, \beta \in V$.

We also check two more useful facts about $E_{c}$.

Lemma 2.4. $E_{c}\left(\beta^{\vee}, \beta\right)=1$ for all $\beta \in \Phi^{\mathrm{re}}$.

Proof. Since $\beta^{\vee}$ is a positive scaling of $\beta$ and $E_{c}$ is bilinear, we have $E_{c}\left(\beta, \beta^{\vee}\right)=$ $E_{c}\left(\beta^{\vee}, \beta\right)$. Thus $(2.2)$ says that $E_{c}\left(\beta^{\vee}, \beta\right)=\frac{1}{2} K\left(\beta^{\vee}, \beta\right)=1$ for $\beta \in \Phi^{\text {re }}$.

Lemma 2.5. $E_{c}(\alpha, \beta)=E_{c^{-1}}(\beta, \alpha)$ for all $\alpha, \beta \in V$.

Proof. For any simple co-root $\alpha_{i}^{\vee}$ and simple root $\alpha_{j}$, we have

$$
E_{c^{-1}}\left(\alpha_{j}, \alpha_{i}^{\vee}\right)=\frac{K\left(\alpha_{j}, \alpha_{j}\right)}{K\left(\alpha_{i}, \alpha_{i}\right)} E_{c^{-1}}\left(\alpha_{j}^{\vee}, \alpha_{i}\right)= \begin{cases}\frac{K\left(\alpha_{j}, \alpha_{j}\right)}{K\left(\alpha_{i}, \alpha_{i}\right)} a_{j i} & \text { if } i>j, \\ 1 & \text { if } i=j, \text { or } \\ 0 & \text { if } i<j,\end{cases}
$$

which agrees with (2.1).

We now review, and slightly modify for the present purposes, a result of Howlett [21, Theorem 2.1]. The proof given here is from [21].

We abuse the notation $E_{c}$ by allowing it to stand not only for the bilinear form as above, but also for the matrix giving that form in the basis of simple roots on the right and simple co-roots on the left. Thus $E_{c}$ is the $n \times n$ matrix whose $i j$-entry is $a_{i j}$ if $i>j$, is 1 if $i=j$, and is 0 if $i<j$. Similarly, $E_{c^{-1}}$ is the $n \times n$ matrix whose $i j$-entry is 0 if $i>j$, is 1 if $i=j$, and is $a_{i j}$ if $i<j$.

Theorem 2.6. Given an arbitrary symmetrizable Cartan matrix $A$ and a Coxeter element $c=s_{1} \cdots s_{n}$, the matrix for $c$ in the basis of simple roots is $-E_{c^{-1}}^{-1} E_{c}$.

Proof. Throughout the proof, the symbol 1 denotes an identity matrix of appropriate size and the symbol 0 denotes a matrix of zeros of appropriate shape. These sizes and shapes are clear from context.

For each $i$ from 1 to $n$, let $\ell_{i}$ and $u_{i}$ be the row vectors such that row $i$ in $A$ is $\left[\begin{array}{lll}\ell_{i} & 2 & u_{i}\end{array}\right]$. In particular, $\ell_{i}$ has $i-1$ entries and $u_{i}$ has $n-i$ entries. Thus the $k^{\text {th }}$ row of $E_{c}$ is $\left[\begin{array}{lll}\ell_{k} & 1 & 0\end{array}\right]$. Similarly, the $k^{\text {th }}$ row of $E_{c^{-1}}$ is $\left[\begin{array}{lll}0 & 1 & u_{k}\end{array}\right]$. The matrix for the simple reflection $s_{k}$ in the basis of simple roots is $\left[\begin{array}{ccc}1 & 0 & 0 \\ -\ell_{k} & -1 & -u_{k} \\ 0 & 0 & 1\end{array}\right]$.

For each $i$ from 1 to $n$, define $L_{i}$ to be the $i \times i$ matrix whose $k^{\text {th }}$ row is $\left[\begin{array}{lll}\ell_{k} & 1 & 0\end{array}\right]$. Define $U_{n-i}$ to be the $(n-i) \times(n-i)$ matrix whose $k^{\text {th }}$ row is $\left[\begin{array}{lll}0 & 1 & u_{i+k}\end{array}\right]$ We now prove by induction on $i$ that for $i=0,1, \ldots, n$ the matrix $E_{c^{-1}} s_{1} \cdots s_{i}$ equals $\left[\begin{array}{cc}-L_{i} & 0 \\ 0 & U_{n-i}\end{array}\right]$. The base case, where $i=0$, says that $E_{c^{-1}}=U_{n}$, which is true by construction. If $i>0$ then by induction $E_{c^{-1}} s_{1} \cdots s_{i}$ is

$$
\left[\begin{array}{cc}
-L_{i-1} & 0 \\
0 & U_{n-i+1}
\end{array}\right] s_{i}=\left[\begin{array}{ccc}
-L_{i-1} & 0 & 0 \\
0 & 1 & u_{i} \\
0 & 0 & U_{n-i}
\end{array}\right]\left[\begin{array}{ccc}
1 & 0 & 0 \\
-\ell_{i} & -1 & -u_{i} \\
0 & 0 & 1
\end{array}\right]=\left[\begin{array}{ccc}
-L_{i-1} & 0 & 0 \\
-\ell_{i} & -1 & 0 \\
0 & 0 & U_{n-i}
\end{array}\right]=\left[\begin{array}{cc}
-L_{i} & 0 \\
0 & U_{n-i}
\end{array}\right] .
$$

This completes the inductive proof. In particular, for $i=n$ we have $E_{c^{-1}} c=-L_{n}$, but by construction $L_{n}=E_{c}$, so $E_{c^{-1}} c=-E_{c}$ as desired.

As a consequence of Theorem 2.6, we obtain two more facts about the form $E_{c}$.

Lemma 2.7. $E_{c}(\alpha, \beta)=-E_{c^{-1}}(\alpha, c \beta)$ for all $\alpha, \beta \in V$.

Proof. Theorem 2.6 says that the matrix describing the form $E_{c}$ in the basis of simple roots on the right and the basis of simple co-roots on the left is $-E_{c^{-1}} c$, where $E_{c^{-1}}$ is the matrix describing the form $E_{c^{-1}}$ in the same bases and $c$ is the matrix describing the action of $c$ in the basis of simple roots (on both sides). 
Lemma 2.8. For $\alpha, \beta \in V$, if $K(\alpha, \beta)=0$, then $E_{c}(\alpha, \beta)=E_{c}\left(\alpha, c^{-1} \beta\right)$.

Proof. Since $K(\alpha, \beta)=0$, we have $E_{c}(\alpha, \beta)=-E_{c}(\beta, \alpha)$ by $(2.2)$. The latter equals $-E_{c^{-1}}(\alpha, \beta)$ by Lemma 2.5 , which equals $E_{c}\left(\alpha, c^{-1} \beta\right)$ by Lemma 2.7 .

A (standard) parabolic subgroup $W^{\prime}$ of a Coxeter group $W$ is a subgroup generated by the reflections in some subset $S^{\prime} \subset S$. The associated (standard) parabolic root subsystem is the set $\Phi^{\prime}=\Phi \cap \operatorname{Span}\left(\left\{\alpha_{i} \in \Phi: s_{i} \in S^{\prime}\right\}\right)$. This is a root system in its own right with simple roots $\left\{\alpha_{i}: s_{i} \in S^{\prime}\right\}$. Given a Coxeter element $c$ of $W$ and $S^{\prime} \subseteq S$, the restriction of $c$ to $W^{\prime}$ is the Coxeter element $c^{\prime}$ of $W^{\prime}$ obtained by taking a reduced word for $c$ and deleting all the letters in $S \backslash S^{\prime}$.

2.2. Affine type. When the symmetric bilinear form $K$ is positive definite, $W$ is finite and $A$ is said to be of finite type. Otherwise, $W$ is infinite.

When $K$ is positive semidefinite and not positive definite and, for all $\Pi^{\prime} \mp \Pi$, the restriction of $K$ to $\operatorname{Span}\left\{\alpha_{i} \in \Pi^{\prime}\right\}$ is positive definite, $A$ is of affine type, and $W$ and $\Phi$ are called affine. Both $W$ and $\Phi$ are irreducible when they are affine. Details on affine root systems are found, for example, in [24, 25]. We break with the common practice of taking an affine root system to have rank $n+1$ and, instead, we continue to index simple roots by $\{1, \ldots, n\}$.

When $A$ is of affine type, there exists an index aff $\in\{1, \ldots, n\}$ such that, writing $W_{\text {fin }}$ for the parabolic subgroup of $W$ generated by $S_{\text {fin }}=S \backslash\left\{s_{\text {aff }}\right\}$, the group $W$ is isomorphic to a semidirect product of $W_{\text {fin }}$ with the lattice generated by the simple co-roots $\left\{\alpha_{i}^{\vee}: i \neq \mathrm{aff}\right\}$. There may not be a unique choice of aff, but the choices are equivalent up to diagram automorphisms, and we fix a choice. We call $\alpha_{\text {aff }}$ the affine simple root and $s_{\text {aff }}$ the affine simple reflection.

We write $\Pi_{\text {fin }}$ for $\Pi \backslash\left\{\alpha_{\text {aff }}\right\}$ and $\Phi_{\text {fin }}$ for the corresponding parabolic root subsystem of $\Phi$. This is an indecomposable finite root system. We write $V_{\text {fin }}$ for the subspace of $V$ spanned by $\Pi_{\text {fin }}$. We have $V=V_{\text {fin }} \oplus \operatorname{Span} \alpha_{\text {aff }}$.

There is a standard construction (see for example [25, Proposition 2.1]) that builds an affine root system from an indecomposible finite root system. We call a root system arising in this way a standard affine root system. The standard affine root systems are represented on Table Aff 1 of [24, Chapter 4]. (See also Table 2.1 of the present paper.) Some affine root systems are not standard, but every affine root system $\Phi$ is a rescaling of a unique standard affine root system $\Phi^{\prime}$. That is, each root of $\Phi$ is a positive scaling of a root in $\Phi^{\prime}$ and the Cartan matrices of $\Phi$ and $\Phi^{\prime}$ define the same symmetric bilinear form $K$ on the space $V$. The rescaling factors are necessarily constant on $W$-orbits of roots.

When $\Phi$ is an affine root system, the form $K$ has a one-dimensional kernel, which contains a one-dimensional sublattice of the root lattice. The nonzero elements of this sublattice are the imaginary roots. Let $\delta$ be the positive imaginary root closest to the origin. Because $\delta$ is in the kernel of $K$, it is fixed by $W$. Every real root in $\Phi$ is a positive scaling of $\beta+k \delta$ for some $\beta \in \Phi_{\text {fin }}$ and $k \in \mathbb{Z}$. If $\Phi$ is a standard affine root system, then every root is $\beta+k \delta$ (with no scaling needed).

The imaginary root $\delta$ has strictly positive simple-root coordinates, so in partic-

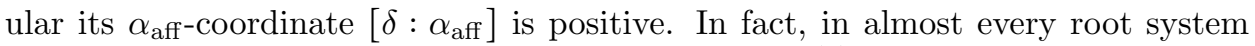
of affine type, $\left[\delta: \alpha_{\text {aff }}\right]=1$. The exception is type $A_{2 k}^{(2)}$, where $\left[\delta: \alpha_{\text {aff }}\right]=2$. The vector $\theta=\delta-\left[\delta: \alpha_{\text {aff }}\right] \alpha_{\text {aff }}$ is a positive root in $\Phi_{\text {fin }}$. In the standard affine root systems $\theta$ is the highest root of $\Phi_{\text {fin }}$, but in other affine root systems, it is either the highest root or the highest short root [24, Proposition 6.4]. 
The co-roots $\left\{\beta^{\vee}: \beta \in \Phi^{\mathrm{re}}\right\}$ are the real roots of the dual root system $\Phi^{\vee}$ (the root system associated to the transpose $A^{T}$ of the Cartan matrix $A$.) The Cartan matrices $A$ and $A^{T}$ define the same symmetric bilinear form $K$, so if $\Phi$ is affine, then $\Phi^{\vee}$ is also affine. Let $\delta^{\vee}$ be the positive imaginary root in $\Phi^{\vee}$ that is closest to zero. This is a positive scaling of $\delta$. Indeed, one can calculate that

$$
\delta^{\vee}= \begin{cases}\frac{2}{K\left(\alpha_{\text {aff }}, \alpha_{\text {aff }}\right)} \delta=\frac{2}{K(\theta, \theta)} \delta & \text { if } \Phi \text { is not of type } A_{2 k}^{(2)}, \text { or } \\ \frac{1}{K\left(\alpha_{\text {aff }}, \alpha_{\text {aff }}\right)} \delta=\frac{4}{K(\theta, \theta)} \delta & \text { if } \Phi \text { is of type } A_{2 k}^{(2)} .\end{cases}
$$

The non-uniformity in (2.3) arises for two reasons: First, $\alpha_{\text {aff }}=\delta-\theta$ in every case except type $A_{2 k}^{(2)}$, where $\alpha_{\text {aff }}=\frac{1}{2}(\delta-\theta)$; and second, the index aff ${ }^{\vee}$ for $\Phi^{\vee}$ can be taken to coincide with the index aff for $\Phi$ in every type except type $A_{2 k}^{(2)}$.

2.3. Coxeter elements in affine type. The following proposition is known and not difficult in type $A_{n}^{(1)}$. It follows from [1, Theorem 1.2] in the other types.

Proposition 2.9. If $W$ is an affine Weyl group not of type $A_{n-1}^{(1)}$, then any two Coxeter elements of $W$ are conjugate in $W$. If $W$ is of type $A_{n-1}^{(1)}$, then there is one conjugacy class for each $k \in\{1, \ldots, n-1\}$, represented by the Coxeter element $s_{1} \cdots s_{n}$ with $\left(s_{1} s_{k+1}\right)^{3}=\left(s_{k} s_{n}\right)^{3}=1$ and $\left(s_{i} s_{i+1}\right)^{3}=1$ for $i \neq k, n$. The conjugations can be carried out by a sequence of source-sink moves.

The following is part of [36, Proposition 3.1]. For an example related to this proposition, see [36, Example 1.3].

Proposition 2.10. Let $\Phi$ be an affine root system and let $c$ be a Coxeter element.

(1) c has eigenvalue 1 with algebraic multiplicity 2 and geometric multiplicity 1. The imaginary root $\delta$ is a 1-eigenvector of $c$.

(2) There exists a unique generalized 1-eigenvector $\gamma_{c}$ contained in the subspace $V_{\text {fin }}$ of $V$. (This means that $(c-1) \gamma_{c}=\delta$.)

(3) c has finite order on the hyperplane $U^{c}=\left\{v \in V: K\left(\gamma_{c}, v\right)=0\right\}$.

Denote by $\phi_{c}$ the element of $V^{*}$ defined by $\left\langle\phi_{c}, v\right\rangle=K\left(\gamma_{c}, v\right)$ for all $v \in V$. In view of Proposition 2.10(3) it is useful to know $\phi_{c}$ up to scaling. The following lemma is a concatenation of [36, Lemma 3.5] and [36, Lemma 3.6].

Lemma 2.11. $\phi_{c}$ is a negative scalar times $\sum_{1 \leq i<j \leq n}\left(\left[\delta: \alpha_{j}\right] a_{i j} \rho_{i}-\left[\delta: \alpha_{i}\right] a_{j i} \rho_{j}\right)$.

We now summarize some results from [36] concerning the $c$-orbits of roots in an affine root system. Let $\Upsilon^{c}$ be $\Phi \cap U^{c}$ and define $\Upsilon_{\text {fin }}^{c}$ to be $\Phi_{\text {fin }} \cap U^{c}$. Let $\Xi_{\text {fin }}^{c}$ be the unique simple system of $\Upsilon_{\text {fin }}^{c}$ such that $\Xi^{c} \subset \Phi^{+}$. The following is a rephrasing of [36, Proposition 4.4].

Proposition 2.12. For any affine root system $\Phi$ and a Coxeter element $c$

(1) $\Upsilon_{\text {fin }}^{c}$ is a finite root system of rank $n-2$.

(2) The irreducible components of $\Upsilon_{\text {fin }}^{c}$ are all of finite type $A$.

(3) $\Xi_{\text {fin }}^{c}=\left\{\beta_{i}\right\}_{i=1}^{n-2}$ can be ordered so that either $c \beta_{i}=\beta_{i+1}$ or $c \beta_{i} \notin \Xi_{\text {fin }}^{c}$.

(4) The order in (3) may not be unique but $\Omega^{c}=\left\{\beta_{1}, t_{\beta_{1}} \beta_{2}, \ldots, t_{\beta_{1}} \cdots t_{\beta_{n-2}} \beta_{n-2}\right\}$ is the same for any choice. 
The set $\Upsilon^{c}$ is not a root system in the usual sense, but in accordance with [33, Theorem 2.7] (which is a theorem of [12] and [14]), it inherits from $\Phi$ a canonical system $\Xi^{c}$ of simple roots. Specifically, $\Xi^{c}$ is the unique minimal subset of $\Upsilon^{c} \cap$ $\Phi^{+}$containing $\Upsilon^{c} \cap \Phi^{+}$in its nonnegative span. See also Remark 3.9 and [36, Remark 1.7].

For a reduced word $c=s_{1} \ldots s_{n}$, let $\psi_{c ; j}^{\rightarrow}=s_{1} \cdots s_{j-1} \alpha_{j}$ and $\psi_{c ; j}^{\leftarrow}=s_{n} \cdots s_{j+1} \alpha_{j}$. Define the sets $\vec{\Psi}^{c}=\left\{\psi_{c ; j}: j=1, \ldots, n\right\}$ and $\overleftarrow{\Psi}^{c}=\left\{\psi_{c ; j}^{\leftarrow}: j=1, \ldots, n\right\}$, and let $\Psi^{c}=$ $\vec{\Psi}^{c} \cap \overleftarrow{\Psi}^{c}$. Since different reduced words for the same Coxeter element are related by a sequence of commutations of adjacent commuting letters, the sets $\vec{\Psi}^{c}, \overleftarrow{\Psi}^{c}$ and $\Psi^{c}$ depend only on $c$, not on the chosen reduced word.

Let $\Omega^{c}$ be as defined in Proposition 2.12. For any $\beta \in \Omega^{c}$, there exists a smallest positive integer $\kappa(\beta)$ such that $\kappa(\beta) \delta-\beta$ is a root. The following is [36, Theorem 1.2]. (A similar result is [13, Proposition 1.9], but the result from [36] has additional details that are crucial for our purposes. See [36, Remark 1.9].)

Theorem 2.13. Suppose $\Phi$ is an affine root system and $c$ is a Coxeter element in the associated Weyl group $W$.

(1) There are exactly $2 n$ infinite c-orbits in $\Phi$. The set $\Psi^{c}$ is a transversal of these orbits.

(2) The c-orbit of a root $\beta \in \Phi$ is finite if and only if $\beta \in U^{c}$.

(3) Every imaginary root is fixed by $c$.

(4) For $\Phi$ of rank 2, there are no finite c-orbits of real roots. For larger rank, there are infinitely many finite c-orbits of real roots and the set $\left\{\beta+m \cdot \kappa(\beta) \delta: \beta \in \Omega^{c}, m \in \mathbb{Z}\right\}$ is a transversal of them.

(5) Each finite $c$-orbit contains either only positive roots or only negative roots. In particular, the c-orbit of a real root $\beta+m \cdot \kappa(\beta) \delta$ for $\beta \in \Omega^{c}$ consists of positive roots if and only if $m \geq 0$.

(6) A finite c-orbit intersects $\Phi_{\text {fin }}^{+}$if and only if it intersects $\Omega^{c}$.

Table 2.1 shows the roots $\beta_{i}$ (indexed as in Proposition 2.12) for standard affine root systems and a particular choice of $c$. (This table also appears as [36, Table 1].) The types are named as in [24, §4.8], except that, as mentioned earlier, we use $n$ as the rank of the root system in every case. The Coxeter element $c=s_{1} \cdots s_{n}$ is described by the labeling of nodes in the second column. In every case, $\alpha_{\text {aff }}=\alpha_{n}$.

The following is [36, Proposition 4.3].

Proposition 2.14. The orbits of roots in $\vec{\Psi}^{c}$ are separated from the orbits of roots in $\overleftarrow{\Psi}^{c}$ by the hyperplane $U^{c}$. Specifically, $K\left(\gamma_{c}, \beta\right)>0$ for $\beta \in c^{m} \vec{\Psi}^{c}$ and $m \in \mathbb{Z}$ while $K\left(\gamma_{c}, \beta\right)<0$ for $\beta \in c^{m} \overleftarrow{\Psi}^{c}$ and $m \in \mathbb{Z}$

Proposition 2.12(2) implies that the irreducible components of $\Upsilon^{c}$ are of affine type $A$. The following is [36, Proposition 4.6].

Proposition 2.15. The action of $c$ on each component of $\Upsilon^{c}$ is to rotate the Dynkin diagram of the component, taking each node to an adjacent node (or when the component has rank 2, to transpose the two nodes of the Dynkin diagram).

We now prove some special facts about the form $E_{c}$ in affine type.

Proposition 2.16. Suppose $\Phi$ is of affine type and $\beta$ is a root contained in $U^{c}$. Then $E_{c}\left(\beta^{\vee}, \delta\right)=E_{c}\left(\delta^{\vee}, \beta\right)=0$. 
Proof. Since $E_{c}\left(\delta^{\vee}, \delta\right)=E_{c}\left(\delta, \delta^{\vee}\right)$ and also $K\left(\delta^{\vee}, \delta\right)=0,(2.2)$ implies $E_{c}\left(\delta^{\vee}, \delta\right)=$ $E_{c}\left(\delta, \delta^{\vee}\right)=0$. We check that $E_{c}\left(\delta^{\vee}, \beta\right)=0$ whenever $\beta$ is a simple root of $\Upsilon^{c}$. We apply Lemma 2.3 repeatedly and recall that $\delta$ is fixed by the action of $W$ to conclude that $E_{c}\left(\delta^{\vee}, \beta^{\prime}\right)$ is constant for roots $\beta^{\prime}$ in the $c$-orbit of $\beta$. The sum over the $c$-orbit of $\beta$ is $\kappa(\beta) \delta$, so the sum of the terms $E_{c}\left(\delta^{\vee}, \beta^{\prime}\right)$ over roots $\beta^{\prime}$ in the $c$-orbit of $\beta$ is $\kappa(\beta) E_{c}\left(\delta^{\vee}, \delta\right)=0$. Thus each of these terms is zero, and in particular $E_{c}\left(\delta^{\vee}, \beta\right)=0$. By linearity $E_{c}\left(\delta^{\vee}, \beta\right)=0$ for any $\beta \in U^{c}$, and since $K\left(\delta^{\vee}, \beta\right)=0$

\begin{tabular}{|c|c|c|c|}
\hline Type & Diagram of $\Phi$ & Diagram of $\Upsilon_{\text {fin }}^{c}$ & Simple roots of $\Upsilon_{\text {fin }}^{c}$ \\
\hline$A_{1}^{(1)}$ & $\left.{ }_{1} \ll\right\rangle_{2}$ & & \\
\hline $\begin{array}{c}A_{n-1}^{(1)} \\
(n \geq 3) \\
k \neq n\end{array}$ & & 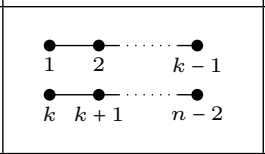 & $\beta_{j}=\alpha_{j+1}$ \\
\hline $\begin{array}{l}B_{n-1}^{(1)} \\
(n \geq 4)\end{array}$ & 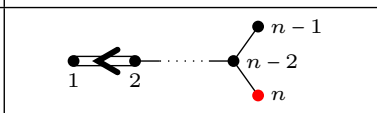 & 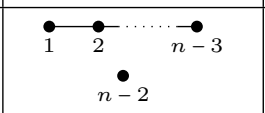 & $\begin{array}{l}\beta_{n-2}=\sum_{i=1}^{n-1} \alpha_{i} \\
\beta_{j}=\alpha_{j+1}\end{array}$ \\
\hline $\begin{array}{l}C_{n-1}^{(1)} \\
(n \geq 3)\end{array}$ & $\ddot{i}>_{2}-\vec{n}_{n-1}<_{n}$ & $\stackrel{0}{i}-\cdots \cdots \cdot \overrightarrow{n-2}$ & $\beta_{j}=\alpha_{j+1}$ \\
\hline $\begin{array}{l}D_{n-1}^{(1)} \\
(n \geq 5)\end{array}$ & . & 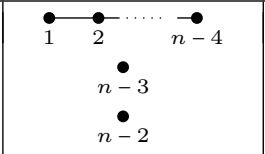 & $\begin{array}{l}\beta_{n-3}=\alpha_{1}+\sum_{i=3}^{n-1} \alpha_{i} \\
\beta_{n-2}=\alpha_{2}+\sum_{i=3}^{n-1} \alpha_{i} \\
\beta_{j}=\alpha_{j+2}\end{array}$ \\
\hline$E_{6}^{(1)}$ & 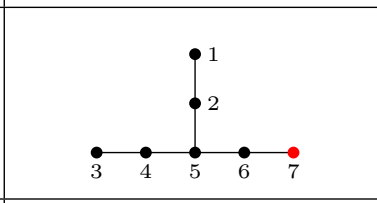 & $\begin{array}{ll}: & 2 \\
\bullet & : \\
3 & 4 \\
5\end{array}$ & $\begin{array}{l}\beta_{1}=\alpha_{4}+\alpha_{5} \\
\beta_{2}=\alpha_{1}+\alpha_{2}+\alpha_{5}+\alpha_{6} \\
\beta_{3}=\alpha_{2}+\alpha_{5} \\
\beta_{4}=\alpha_{3}+\alpha_{4}+\alpha_{5}+\alpha_{6} \\
\beta_{5}=\alpha_{2}+\alpha_{4}+\alpha_{5}+\alpha_{6}\end{array}$ \\
\hline$E_{7}^{(1)}$ & $\stackrel{0}{2}:$ & 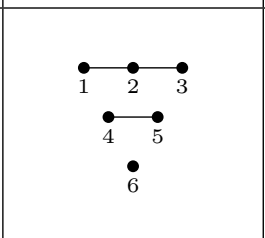 & $\begin{array}{l}\beta_{1}=\alpha_{4}+\alpha_{5} \\
\beta_{2}=\alpha_{1}+\alpha_{5}+\alpha_{6} \\
\beta_{3}=\sum_{i=2}^{7} \alpha_{i} \\
\beta_{4}=\sum_{i=3}^{6} \alpha_{i} \\
\beta_{5}=\alpha_{1}+\sum_{i=4}^{7} \alpha_{i} \\
\beta_{6}=\alpha_{1}+\alpha_{5}+\sum_{i=3}^{7} \alpha_{i}\end{array}$ \\
\hline$E_{8}^{(1)}$ & 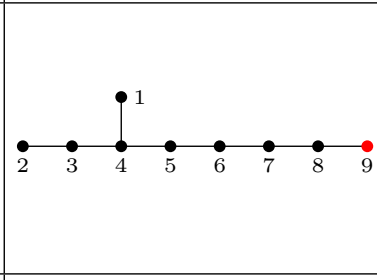 & 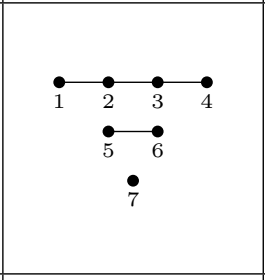 & $\begin{array}{l}\beta_{1}=\alpha_{3}+\alpha_{4}+\alpha_{5} \\
\beta_{2}=\alpha_{1}+\sum_{i=4}^{6} \alpha_{i} \\
\beta_{3}=\sum_{i=2}^{7} \alpha_{i} \\
\beta_{4}=\alpha_{1}+\sum_{i=3}^{8} \alpha_{i} \\
\beta_{5}=\alpha_{1}+\alpha_{4}+\sum_{i=3}^{7} \alpha_{i} \\
\beta_{6}=\alpha_{4}+\alpha_{5}+\sum_{i=1}^{8} \alpha_{i} \\
\beta_{7}=\alpha_{4}+\sum_{i=3}^{6} \alpha_{i}+\sum_{i=1}^{8} \alpha_{i}\end{array}$ \\
\hline$F_{4}^{(1)}$ & 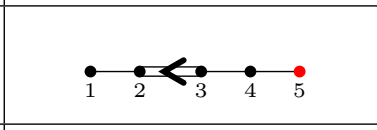 & $\stackrel{\bullet}{i} \dot{2}$ & $\begin{array}{l}\beta_{1}=\alpha_{2}+\alpha_{3} \\
\beta_{2}=\alpha_{1}+\alpha_{2}+\alpha_{3}+\alpha_{4} \\
\beta_{3}=2 \alpha_{2}+\alpha_{3}+\alpha_{4}\end{array}$ \\
\hline$G_{2}^{(1)}$ & $\underset{1}{\rightleftarrows}$ & $i$ & $\beta_{1}=\alpha_{1}+\alpha_{2}$ \\
\hline
\end{tabular}

TABLE 2.1. Standard affine root systems and their finite orbits 
for any $\operatorname{root} \beta,(2.2)$ implies that $E_{c}\left(\beta, \delta^{\vee}\right)=0$ as well, and thus $E_{c}\left(\beta^{\vee}, \delta\right)=0$ by linearity.

Proposition 2.17. Suppose $\Phi$ is of affine type and $\beta, \beta^{\prime}$ are simple roots of the root subsystem $\Upsilon^{c}$ of $\Phi$. Then

$$
E_{c}\left(\beta^{\vee}, \beta^{\prime}\right)=\left\{\begin{aligned}
1 & \text { if } \beta^{\prime}=\beta, \\
-1 & \text { if } \beta^{\prime}=c^{-1} \beta, \text { or } \\
0 & \text { otherwise. }
\end{aligned}\right.
$$

Proof. Lemma 2.4 says that $E_{c}\left(\beta^{\vee}, \beta\right)=1$. Lemma 2.7 says that $E_{c}\left(\beta^{\vee}, c^{-1} \beta\right)=$ $-E_{c^{-1}}\left(\beta^{\vee}, \beta\right)=-1$. If $\beta$ and $\beta^{\prime}$ are in different components of $\Upsilon^{c}$, then $K\left(\beta^{\vee}, \beta^{\prime}\right)=0$ and thus Lemma 2.8 says that $E_{c}\left(\beta^{\vee}, \beta^{\prime}\right)=E_{c}\left(\beta^{\vee}, c^{-1} \beta^{\prime}\right)$. But then $c^{-1} \beta^{\prime}$ is in the same component as $\beta^{\prime}$, so we can continue to show that $E_{c}\left(\beta^{\vee}, c^{-1} \beta^{\prime}\right)=$ $E_{c}\left(\beta^{\vee}, c^{-2} \beta^{\prime}\right)$, and so forth until we conclude that $E_{c}\left(\beta^{\vee}, \beta^{\prime}\right)=E_{c}\left(\beta^{\vee}, c^{k} \beta^{\prime}\right)$ is constant as $k$ varies. The sum over the $c$-orbit of $\beta^{\prime}$ is $\kappa(\beta) \delta$, and since $E_{c}\left(\beta^{\vee}, \delta\right)=0$ by Proposition 2.16, we see that $E_{c}\left(\beta^{\vee}, \beta^{\prime}\right)=0$.

It remains to show that $E_{c}\left(\beta^{\vee}, \beta^{\prime}\right)=0$ when $\beta^{\prime}$ is in the $c$-orbit of $\beta$ but $\beta^{\prime} \notin$ $\left\{\beta, c^{-1} \beta\right\}$. The $c$-orbit of $\beta$ has finite size $k \geq 2$. If $k=2$, then we are done, so assume $k>2$. We argue by induction on $i=2, \ldots, k-1$ that $E_{c}\left(\beta^{\vee}, c^{-i} \beta\right)=0$. For the base case $i=2$, by replacing $c$ by $c^{-1}$ in the statement $E_{c}\left(\beta^{\vee}, c^{-1} \beta\right)=-1$ which we already proved, we obtain $E_{c^{-1}}\left(\beta^{\vee}, c \beta\right)=-1$. Then Lemma 2.3 says that $E_{c^{-1}}\left(c^{-1} \beta^{\vee}, \beta\right)=-1$. Since $K\left(c^{-1} \beta^{\vee}, \beta\right)=-1,(2.2)$ implies that $E_{c^{-1}}\left(\beta, c^{-1} \beta^{\vee}\right)=0$. By bilinearity, $E_{c^{-1}}\left(\beta^{\vee}, c^{-1} \beta\right)=0$ as well, so $E_{c}\left(\beta^{\vee}, c^{-2} \beta\right)=0$ by Lemma 2.7. If $2<i \leq k-1$ then by induction $E_{c}\left(\beta^{\vee}, c^{-i+1} \beta\right)=0$. Since $K\left(\beta^{\vee}, c^{-i+1} \beta\right)=0$, we appeal to Lemma 2.8 to conclude that $E_{c}\left(\beta^{\vee}, c^{-i} \beta\right)=E_{c}\left(\beta^{\vee}, c^{-i+1} \beta\right)=0$.

\section{The ROOTS $\Phi_{c}$}

Definition 3.1. Suppose $\Phi$ is an affine root system and $c$ is a Coxeter element. We write $\Lambda_{c}^{\text {re }}=\left\{c^{k} \beta: k \in \mathbb{Z}, \beta \in \Omega^{c}\right\}$. This is finite by Theorem 2.13. We write $\Lambda_{c}$ for $\Lambda_{c}^{\text {re }} \cup\{\delta\}$. We define

$$
\begin{aligned}
\Phi_{c} & =-\Pi \cup\left(\Phi^{+} \backslash U^{c}\right) \cup \Lambda_{c}, \\
\Phi_{c}^{\mathrm{re}} & =-\Pi \cup\left(\Phi^{+} \backslash U^{c}\right) \cup \Lambda_{c}^{\mathrm{re}}=\Phi_{c} \backslash\{\delta\} .
\end{aligned}
$$

Since $\Omega^{c}=\Omega^{c^{-1}}$ and $U^{c}=U^{c^{-1}}$, we have the following proposition:

Proposition 3.2. $\Phi_{c}=\Phi_{c^{-1}}$.

For any simple reflection $s \in S$ denote by $\alpha_{s} \in \Pi$ the simple root associated to $s$ and define a map $\sigma_{s}$ on $-\Pi \cup \Phi^{+}$by

$$
\sigma_{s}(\alpha)= \begin{cases}\alpha & \text { if } \alpha \in-\Pi \backslash\left\{-\alpha_{s}\right\} \\ s(\alpha) & \text { otherwise }\end{cases}
$$

Proposition 3.3. The map $\sigma_{s}$ is an involution on $-\Pi \cup \Phi^{+}$. If $s$ is initial or final in $c$, then $\sigma_{s}$ restricts to a bijection from $\Phi_{c}$ to $\Phi_{s c s}$.

The proof of Proposition 3.3 uses a few lemmas. The first is [36, Corollary 3.3].

Lemma 3.4. If $s$ is initial or final in $c$, then $U^{s c s}=s U^{c}$.

Lemma 3.5. If $s$ is initial or final in $c$, then $\alpha_{s} \notin \Upsilon^{c}$. 
Proof. Since $s$ is initial or final in $c$, either $c \alpha_{s}$ or $c^{-1} \alpha_{s}$ is a negative root, and thus Theorem 2.13 lets us rule out the possibility that $\alpha_{s} \in \Upsilon^{c}$.

Lemma 3.6. If $s$ is initial or final in $c$, then the simple roots of $\Upsilon^{c}$ and of $\Upsilon^{s c s}$ are related by $\Xi^{s c s}=s \Xi^{c}$, or equivalently, $\Xi^{s c s}=\sigma_{s} \Xi^{c}$.

Proof. Lemma 3.4 says that $U^{s c s}=s U^{c}$. Since $\Upsilon^{c}$ is the set of roots in $\Phi$ that are contained in $U^{c}$, and similarly for $\Upsilon^{s c s}$, and since $\pm \alpha_{s}$ are the only roots that change sign under the action of $s$, and $\alpha_{s} \notin \Upsilon^{c}$ by Lemma 3.5, the simple roots $\Xi^{s c s}$ are obtained from the simple roots $\Xi^{c}$ by the action of $s$. Since all of these roots are positive, the action of $s$ on them corresponds to the action of $\sigma_{s}$.

Definition 3.7. The support $\operatorname{Supp}_{\Pi}(\beta)$ of a $\operatorname{root} \beta$ is the set of simple roots that contribute with non-zero coefficient to the simple root expansion of $\beta$. The support $\operatorname{Supp}_{\Pi}(R)$ of a set $R$ of roots is $\bigcup_{\beta \in R} \operatorname{Supp}_{\Pi}(\beta)$. A support is $f u l l$ if it is the entire set $\Pi$ of simple roots.

Definition 3.8. Given a real root $\beta \in \Upsilon^{c}$, its tube support $\operatorname{Supp}_{\Xi}(\beta)$ is the support of $\beta$ as a root in $\Upsilon^{c}$ (the set of roots that appear with nonzero coefficients in the expansion of $\beta$ as a linear combination of roots in $\Xi^{c}$ ). The tube support of a set $R \subseteq \Upsilon^{c}$ of real roots is $\operatorname{Supp}_{\Xi}(R)=\bigcup_{\beta \in R} \operatorname{Supp}_{\Xi}(\beta)$. A tube support is component-full if it contains the full set of simple roots in some component of $\Upsilon^{c}$.

Remark 3.9. The simple roots $\Xi^{c}$ of $\Upsilon^{c}$ may fail to be a basis for their linear span, precisely to the extent that $\Upsilon^{c}$ is reducible. However, every real root in $\Upsilon^{c}$ is in some irreducible component of $\Upsilon^{c}$ and thus has a unique expansion as a linear combination of simple roots. We do not consider $\operatorname{Supp}_{\Xi}(\delta)$, which is not well-defined when $\Upsilon^{c}$ is reducible. For a set $R$ of real roots in $\Upsilon^{c}$, the tube support $\operatorname{Supp}_{\Xi}(R)$ may contain simple roots in multiple components of $\Upsilon^{c}$. It is component-full if it contains the entire set of simple roots in at least one of them.

Lemma 3.10. The set $\Lambda_{c}^{\mathrm{re}}$ is the set of positive real roots of $\Upsilon^{c}$ whose tube support is not component-full.

Proof. This is immediate from Proposition 2.12(2) and Proposition 2.15.

Lemma 3.11. If $s$ is initial or final in $c$, then $\Lambda_{s c s}^{\mathrm{re}}=\sigma_{s}\left(\Lambda_{c}^{\mathrm{re}}\right)$.

Proof. This is an immediate consequence of Lemmas 3.6 and 3.10 .

Proof of Proposition 3.3. The first assertion is immediate because $\alpha_{s}$ is the unique positive root whose sign is changed by $s$. By the symmetry of swapping $c$ and $s c s$, it is enough to show that $\sigma_{s}\left(\Phi_{c}\right)=\Phi_{s c s}$. The map $\sigma_{s}$ fixes $-\Pi \backslash\left\{-\alpha_{s}\right\}$, swaps $\pm \alpha_{s}$, and sends all positive roots, aside from $\alpha_{s}$, to positive roots. It also fixes $\delta$. Lemmas 3.4, 3.10 and 3.11 complete the proof.

Define $\tau_{c}=\sigma_{1} \cdots \sigma_{n}$ where $\sigma_{i}$ is an abbreviation for $\sigma_{s_{i}}$.

Proposition 3.12. Suppose $\Phi$ is an affine root system, $c=s_{1} \cdots s_{n}$ is a Coxeter element in the associated Weyl group $W$, and $\tau_{c}$ is $\sigma_{1} \cdots \sigma_{n}$ as above.

(1) The map $\tau_{c}$ restricts to a permutation of $\Phi_{c}$.

(2) For $\alpha \in \Phi_{c}$,

$$
\tau_{c}(\alpha)=\left\{\begin{array}{ll}
\psi_{c ; i} & \text { if } \alpha=-\alpha_{i} \\
-\alpha_{i} & \text { if } \alpha=\psi_{c ; i}^{\leftarrow} \\
c \alpha & \text { otherwise }
\end{array} \quad \text { and } \quad \tau_{c}^{-1}(\alpha)= \begin{cases}\psi_{c ; i}^{\leftarrow} & \text { if } \alpha=-\alpha_{i} \\
-\alpha_{i} & \text { if } \alpha=\psi_{c ; i} \\
c^{-1} \alpha & \text { otherwise. }\end{cases}\right.
$$


(3) There are $n$ infinite $\tau_{c}$-orbits and $n-2$ finite $\tau_{c}$-orbits in $\Phi_{c}^{\mathrm{re}}$.

(4) The set $-\Pi$ is a transversal of the infinite $\tau_{c}$-orbits in $\Phi_{c}$.

(5) A root $\alpha \in \Phi_{c}$ is in a finite $\tau_{c^{-}}$orbit if and only if $\alpha \in \Phi^{+} \cap U^{c}$.

(6) $\Omega^{c}$ is a transversal of the finite $\tau_{c}$-orbits in $\Phi_{c}^{\mathrm{re}}$.

(7) $\{\delta\}$ is a $\tau_{c}$-orbit.

(8) For all $i, K\left(\gamma_{c}, \tau_{c}^{m}\left(-\alpha_{i}\right)\right)>0$ if $m>0$ and $K\left(\gamma_{c}, \tau_{c}^{m}\left(-\alpha_{i}\right)\right)<0$ if $m<0$.

Proof. The first assertion is obtained by applying Proposition $3.3 n$ times. The expressions in (2) are readily verified by inspection. Assertions (3)-(7) follow from (2) and Theorem 2.13. Proposition 2.14 and (2) imply (8).

The properties of $\tau_{c}$-orbits given in Proposition 3.12 allow us to give several additional characterizations of $\Phi_{c}$.

Proposition 3.13. Each of the following expressions specifies the set $\Phi_{c}^{\mathrm{re}}$. (The symbol $\sqcup$ is disjoint union.)

(1) $\left\{\tau_{c}^{k} \beta: \beta \in\left(-\Pi \cup \Omega^{c}\right), k \in \mathbb{Z}\right\}$.

(2) $\left\{c^{-m} \psi_{c ; j}^{\leftarrow}: m \geq 0,1 \leq j \leq n\right\} \cup-\Pi \sqcup\left\{c^{m} \psi_{c ; j}^{\rightarrow}: m \geq 0,1 \leq j \leq n\right\} \cup \Lambda_{c}^{\mathrm{re}}$.

(3) The union of all finite $\tau_{c}$-orbits of roots $\beta \in \Phi_{\text {fin }}^{+}$and all infinite $\tau_{c}$-orbits.

(4) $\left\{c^{k} \beta \in \Phi: \operatorname{Supp}_{\Pi}(\beta) \mp \Pi, k \in \mathbb{Z}\right\} \cap\left(\Phi^{+} \cup-\Pi\right)$.

(5) $\left\{\tau_{c}^{k} \beta \in \Phi^{+}: \operatorname{Supp}_{\Pi}(\beta) \mp \Pi, k \in \mathbb{Z}\right\}$.

Proof. Characterization (1) is a direct restatement of Proposition 3.12(4,6). Characterization (2) also follows easily from Proposition 3.12.

Observe that any root $\beta$ in $\Phi^{+} \backslash \Phi_{c}$ has $\operatorname{Supp}_{\Pi}(\beta)=\Pi$; indeed by Theorem 2.13(4,5) all the real roots $\beta$ in $\Phi^{+} \backslash \Phi_{c}$ are of the form $\beta=c^{k} \beta^{\prime}+m \cdot \kappa\left(\beta^{\prime}\right) \delta$ for some positive root $c^{k} \beta^{\prime} \in \Lambda_{c}^{\text {re }}\left(\right.$ with $\left.\beta^{\prime} \in \Omega^{c}\right)$ and $m>0$. In particular $\operatorname{Supp}_{\Pi}(\beta) \supseteq$ $\operatorname{Supp}_{\Pi}(\delta)=\Pi$ so that $\beta \notin \Phi_{\text {fin }}$. Characterization (3) then follows immediately from the inclusion $\Omega^{c} \mp \Phi_{\text {fin }}$.

By the same observation, a root $\beta \in \Phi_{c}$ is in a finite $c$ - (or equivalently $\tau_{c^{-}}$) orbit if and only if it is in the orbit of some $\beta^{\prime} \in \Omega^{c} \subset \Phi_{\text {fin. }}$. Such a $\beta^{\prime}$ has $\operatorname{Supp}_{\Pi}\left(\beta^{\prime}\right) \mp \Pi$, so for Characterizations (4) and (5) it remains only to consider infinite orbits.

The only roots in $\Psi^{c}$ that can possibly have full support are $s_{1} \cdots s_{n-1} \alpha_{n}$ and $s_{n} \cdots s_{2} \alpha_{1}$. We have $c s_{1} \cdots s_{n-1} \alpha_{n}=-\alpha_{n}$ and $c^{-1} s_{n} \cdots s_{2} \alpha_{1}=-\alpha_{1}$, establishing Characterization (4). We also have $\tau_{c}^{2} s_{1} \cdots s_{n-1} \alpha_{n}=\alpha_{n}$ and $\tau_{c}^{-2} s_{n} \cdots s_{2} \alpha_{1}=\alpha_{1}$, proving Characterization (5).

Remark 3.14. The set $\Phi_{c}$ is an affine version of the set $\Phi_{\geq-1}$ of almost positive roots in a root system $\Phi$ of finite type. We deviate from the standard convention and denote the latter also by $\Phi_{c}$ even though this set does not depend on the choice of Coxeter element. This notation allows us treat the finite and affine cases together in several definitions and proofs.

Proposition 3.15. If $\Phi$ is of affine type and $\Phi^{\prime}$ is a parabolic root subsystem of $\Phi$, then $\Phi_{c} \cap \Phi^{\prime}=\Phi_{c^{\prime}}^{\prime}$ where $c^{\prime}$ is the restriction of $c$ to the parabolic subgroup $W^{\prime}$.

Proof. If $\Phi^{\prime}$ is a proper parabolic root subsystem of $\Phi$, then $\Phi^{\prime}$ is finite because $\Phi$ is affine. Thus the assertion follows from Proposition 3.13(5). 


\section{The COMPAtibility DEGREE}

In this section, we define the compatibility degree for ordered pairs roots in $\Phi_{c}$ and establish its crucial properties. We need all of the notation of the previous sections. Reminders on notation are found in the Index to Notation on page 43.

Definition 4.1. Suppose $\alpha \in \Upsilon^{c}$ is a root in the subsystem $\Phi \cap U^{c}$ and suppose $\beta_{j} \in \Xi^{c}$ (i.e. $\beta_{j}$ is a simple root of the subsystem). Then $\beta_{j}$ is adjacent to $\alpha$ if $\beta_{j}$ is in $\operatorname{Supp}_{\Xi}(c \alpha) \cup \operatorname{Supp}_{\Xi}\left(c^{-1} \alpha\right)$ but not in $\operatorname{Supp}_{\Xi}(\alpha)$. Given $\alpha, \beta \in \Upsilon^{c}$, define $\operatorname{adj}_{\alpha}(\beta)$ to be the number of roots of $\Xi^{c}$ that are adjacent to $\alpha$ and contained in $\operatorname{Supp}_{\Xi}(\beta)$. This number is not symmetric in $\alpha$ and $\beta$. Define also

$$
(\alpha \circlearrowright \beta)_{c}=\left\{\begin{aligned}
-1 & \text { if } \alpha=\beta, \\
0 & \text { if } \operatorname{Supp}_{\Xi}(\alpha) \mp \operatorname{Supp}_{\Xi}(\beta) \text { or } \operatorname{Supp}_{\Xi}(\beta) \mp \operatorname{Supp}_{\Xi}(\alpha), \\
\operatorname{adj}_{\alpha}(\beta) & \text { otherwise. }
\end{aligned}\right.
$$

Remark 4.2. Recall from Proposition 2.12(2) and from Proposition 2.15 that the irreducible components of $\Upsilon^{c}$ are of affine type $A$ and that $c$ acts on a component by rotating its Dynkin diagram. The tube $\operatorname{support} \operatorname{Supp}_{\Xi}(\alpha)$ of a root $\alpha \in \Upsilon^{c}$ is a connected subgraph of one of these components. Thus $\beta_{j}$ is adjacent to $\alpha$ if and only if $\beta_{j}$ is not in $\operatorname{Supp}_{\Xi}(\alpha)$ but is connected to $\operatorname{Supp}_{\Xi}(\alpha)$ by an edge in the Dynkin diagram of $\Upsilon^{c}$.

We continue to denote by $\left[\beta: \alpha_{i}\right]$ the $\alpha_{i}$-coordinate of $\beta$ in the basis of simple roots and set $\left[\beta^{\vee}: \alpha_{i}^{\vee}\right]$ to be the $\alpha_{i}^{\vee}$-coordinate of $\beta^{\vee}$ in the basis of simple co-roots. We write $\left[\beta: \alpha_{i}\right]_{+}$for $\max \left(\left[\beta: \alpha_{i}\right], 0\right)$ and $\left[\alpha^{\vee}: \alpha_{i}^{\vee}\right]_{+}$for $\max \left(\left[\alpha^{\vee}: \alpha_{i}^{\vee}\right], 0\right)$. For roots $\alpha$ and $\beta$ in $\Phi_{c}$, define

$$
\begin{aligned}
& (\alpha \rightarrow \beta)_{c}=-\sum_{i=1}^{n}\left[\alpha^{\vee}: \alpha_{i}^{\vee}\right]\left[\beta: \alpha_{i}\right]-\sum_{1 \leq j<i \leq n} a_{i j}\left[\alpha^{\vee}: \alpha_{i}^{\vee}\right]_{+}\left[\beta: \alpha_{j}\right]_{+} \\
& (\alpha \leftarrow \beta)_{c}=-\sum_{i=1}^{n}\left[\alpha^{\vee}: \alpha_{i}^{\vee}\right]\left[\beta: \alpha_{i}\right]-\sum_{1 \leq i<j \leq n} a_{i j}\left[\alpha^{\vee}: \alpha_{i}^{\vee}\right]_{+}\left[\beta: \alpha_{j}\right]_{+} \cdot
\end{aligned}
$$

Definition 4.3. For $\alpha$ and $\beta$ in $\Phi_{c}$, the c-compatibility degree of $\alpha$ with $\beta$ is

$$
(\alpha \| \beta)_{c}=\left\{\begin{array}{lc}
\max \left[(\alpha \rightarrow \beta)_{c},(\alpha \leftarrow \beta)_{c}\right] & \text { except when } \alpha, \beta \in \Lambda_{c}^{\text {re }} \\
\operatorname{adj}_{\alpha}(\beta) & \text { and } \operatorname{Supp}_{\Xi}(\alpha, \beta) \text { is component-full. }
\end{array}\right.
$$

Example 4.4. To help the reader parse the definition we compute the $c$-compatibility degree $\left(2 \alpha_{1}+\alpha_{2} \| \alpha_{2}\right)_{c}$ in type $D_{3}^{(2)}$ for $c=s_{1} s_{2} s_{3}$. (Compare this example with [36, Example 1.3] and Example 6.5.) First observe that $2 \alpha_{1}+\alpha_{2}$ is in an infinite $\tau_{c}$-orbit so we are in the first case of Definition 4.3. Since $\left(2 \alpha_{1}+\alpha_{2}\right)^{\vee}=\alpha_{1}^{\vee}+\alpha_{2}^{\vee}$ we have:

$$
\begin{aligned}
& \left(2 \alpha_{1}+\alpha_{2} \rightarrow \alpha_{2}\right)_{c}=-(1 \cdot 0+1 \cdot 1+0 \cdot 0)-(-1 \cdot 1 \cdot 0+0 \cdot 0 \cdot 0-2 \cdot 0 \cdot 0)=-1 \\
& \left(2 \alpha_{1}+\alpha_{2} \leftarrow \alpha_{2}\right)_{c}=-(1 \cdot 0+1 \cdot 1+0 \cdot 0)-(-2 \cdot 1 \cdot 1+0 \cdot 1 \cdot 0-1 \cdot 1 \cdot 0)=1
\end{aligned}
$$

and we get $\left(2 \alpha_{1}+\alpha_{2} \| \alpha_{2}\right)_{c}=1$. For further examples involving pairs of roots in $U^{c}$ we refer the reader to the proof of Theorems 4.5 and 4.6. 
In the next two theorems, we establish the following properties and show that they uniquely characterize the compatibility degree.

$$
\begin{aligned}
\left(-\alpha_{i} \| \beta\right)_{c} & =\left[\beta: \alpha_{i}\right] \text { for } \alpha_{i} \text { simple and } \beta \in \Phi_{c} . \\
\left(\beta \|-\alpha_{i}\right)_{c} & =\left[\beta^{\vee}: \alpha_{i}^{\vee}\right] \text { for } \alpha_{i} \text { simple and } \beta \in \Phi_{c} . \\
(\alpha \| \beta)_{c} & =(\alpha \circlearrowright \beta)_{c} \text { if } \alpha, \beta \in \Lambda_{c}^{\mathrm{re}} . \\
(\delta \| \alpha)_{c} & =(\alpha \| \delta)_{c}=0 \text { if } \alpha \in \Lambda_{c} . \\
(\alpha \| \beta)_{c} & =\left(\sigma_{s} \alpha \| \sigma_{s} \beta\right)_{s c s} \text { for } \alpha, \beta \in \Phi_{c} \text { and } s \text { initial or final in } c . \\
(\alpha \| \beta)_{c} & =\left(\tau_{c} \alpha \| \tau_{c} \beta\right)_{c} .
\end{aligned}
$$

Theorem 4.5. Fix a finite or affine root system $\Phi$. The assignment $(c, \alpha, \beta) \mapsto$ $(\alpha \| \beta)_{c}$ is the unique function satisfying (4.3)-(4.7).

Theorem 4.6. Fix a finite or affine root system $\Phi$ and a Coxeter element $c$. The assignment $(\alpha, \beta) \mapsto(\alpha \| \beta)_{c}$ is the unique function satisfying (4.3)-(4.6) and (4.8).

We now give two results on computing the compatibility degree for special pairs of roots (positive roots in $\Phi_{c}$ or roots in $\Lambda_{c}^{\mathrm{re}}$ ). The first of these results is immediate from the definitions:

Lemma 4.7. If $\alpha$ and $\beta$ are positive roots, then

$$
(\alpha \rightarrow \beta)_{c}=-E_{c}\left(\alpha^{\vee}, \beta\right) \quad \text { and } \quad(\alpha \leftarrow \beta)_{c}=-E_{c^{-1}}\left(\alpha^{\vee}, \beta\right) .
$$

Lemma 4.7 combines with Proposition 2.17 (and the fact that each component of $\Upsilon^{c}$ is of affine type A) to give the following description of $(\alpha \rightarrow \beta)_{c}$ and $(\alpha \leftarrow \beta)_{c}$ when $\alpha$ and $\beta$ are in $\Lambda_{c}^{\text {re }}$. (That is, $\alpha$ and $\beta$ are positive roots in $\Phi_{c}$, contained in finite $\tau_{c}$-orbits.)

Proposition 4.8. If $\alpha, \beta \in \Lambda_{c}^{\mathrm{re}}$, then

(1) $(\alpha \rightarrow \beta)_{c}$ is the number of roots $\beta_{i} \in \operatorname{Supp}_{\Xi}(\alpha)$ with $c \beta_{i} \in \operatorname{Supp}_{\Xi}(\beta)$ minus the number of roots in $\operatorname{Supp}_{\Xi}(\alpha) \cap \operatorname{Supp}_{\Xi}(\beta)$.

(2) $(\alpha \leftarrow \beta)_{c}$ is the number of roots $\beta_{i} \in \operatorname{Supp}_{\Xi}(\alpha)$ with $c^{-1} \beta_{i} \in \operatorname{Supp}_{\Xi}(\beta)$ minus the number of roots in $\operatorname{Supp}_{\Xi}(\alpha) \cap \operatorname{Supp}_{\Xi}(\beta)$.

We now prove Theorems 4.5 and 4.6 .

Proof of Theorems 4.5 and 4.6. Property (4.3) records the simple observation that $\left(\left(-\alpha_{i}\right) \rightarrow \beta\right)_{c}$ and $\left(\left(-\alpha_{i}\right) \leftarrow \beta\right)_{c}$ both equal $\left[\beta: \alpha_{i}\right]$. Property (4.4) is similar.

To verify Property (4.5), we need to show that $\max \left[(\alpha \rightarrow \beta)_{c},(\alpha \leftarrow \beta)_{c}\right]$ equals $(\alpha \circlearrowright \beta)_{c}$ when $\alpha, \beta \in \Lambda_{c}^{\mathrm{re}}$, except possibly when $\operatorname{Supp}_{\Xi}(\alpha, \beta)$ is component-full. This comes down to checking several cases and computing $(\alpha \rightarrow \beta)_{c}$ and $(\alpha \leftarrow \beta)_{c}$ as described in Proposition 4.8. If $\alpha$ and $\beta$ are in different components of $\Upsilon^{c}$, then $(\alpha \rightarrow \beta)_{c}=(\alpha \leftarrow \beta)_{c}=0$, so their maximum is 0 as desired. The remaining cases are described in Table 4.1, with representative pictures. In each case, $\operatorname{Supp}_{\Xi}(\alpha)$ is outlined in red and $\operatorname{Supp}_{\Xi}(\beta)$ is outlined in dotted blue. The quantity $\operatorname{adj}_{\alpha}(\beta)$ is left out of the table when it is irrelevant. The action of $c$ is to rotate counterclockwise by one position. Some of the cases have variations not pictured. For example, in the case where $\operatorname{Supp}_{\Xi}(\alpha)$ and $\operatorname{Supp}_{\Xi}(\beta)$ are nested, their tube supports can have one or the other endpoint in common (or neither, but not both). In these variations, the values of $(\alpha \rightarrow \beta)_{c}$ and $(\alpha \leftarrow \beta)_{c}$ can vary, but their max does not vary and by definition $(\alpha \circlearrowright \beta)_{c}$ does not vary. In the cases where $\operatorname{Supp}_{\Xi}(\alpha)$ and $\operatorname{Supp}_{\Xi}(\beta)$ are nested, $(\alpha \rightarrow \beta)_{c}$ and $(\alpha \leftarrow \beta)_{c}$ may be 0 or -1 , but at least one of them is 0 . 


\begin{tabular}{|c|c|c|c|c|c|c|}
\hline Description & Pictures & $(\alpha \rightarrow \beta)_{c}$ & $(\alpha \leftarrow \beta)_{c}$ & $\operatorname{adj}_{\alpha}(\beta)$ & $(\alpha \circlearrowright \beta)_{c}$ & $(\alpha \| \beta)_{c}$ \\
\hline $\begin{array}{c}\alpha \text { and } \beta \\
\text { disjoint, not } \\
\text { adjacent }\end{array}$ & -1 & -1 & -1 & -1 \\
\hline $\begin{array}{c}\text { Supp }(\alpha) \text { and } \\
\text { Supp } \\
\text { nested }\end{array}$ \\
$\begin{array}{c}\alpha \text { and } \beta \\
\text { overlapping or } \\
\text { adjacent on } \\
\text { one side }\end{array}$
\end{tabular}

TABLE 4.1. Compatibility degree for positive real roots in $U^{c}$

Property (4.6) holds by Proposition 2.16 and Lemma 4.7.

To show that (4.7) holds, first, consider the case where one of $\alpha$ and $\beta$ (or both) is in $-\Pi \backslash\left\{-\alpha_{s}\right\}$. In particular, we know that the exceptional case of the definition of $(\alpha \| \beta)_{c}$ does not apply, so we have to prove that the maximum of $(\alpha \rightarrow \beta)_{c}$ and $(\alpha \leftarrow \beta)_{c}$ equals the maximum of $\left(\sigma_{s} \alpha \rightarrow \sigma_{s} \beta\right)_{s c s}$ and $\left(\sigma_{s} \alpha \leftarrow \sigma_{s} \beta\right)_{s c s}$.

If $\alpha=-\alpha_{i}$, then both $(\alpha \rightarrow \beta)_{c}$ and $(\alpha \leftarrow \beta)_{c}$ equal $\left[\beta: \alpha_{i}\right]$. But $\sigma_{s} \alpha=\alpha=-\alpha_{i}$ so both $\left(\sigma_{s} \alpha \rightarrow \sigma_{s} \beta\right)_{s c s}$ and $\left(\sigma_{s} \alpha \leftarrow \sigma_{s} \beta\right)_{s c s}$ equal $\left[\sigma_{s} \beta: \alpha_{i}\right]$. Since $\sigma_{s} \beta=\beta+a \alpha_{s}$ for some $a$ we have $\left[\sigma_{s} \beta: \alpha_{i}\right]=\left[\beta: \alpha_{i}\right]$. The case where $\beta=-\alpha_{i}$ is similar.

We can now assume that neither $\alpha$ nor $\beta$ is in $-\Pi \backslash\left\{-\alpha_{s}\right\}$. In particular $\sigma_{s}$ acts on $\alpha$ and $\beta$ as $s$, so Lemmas 2.2 and 4.7 combine to prove (4.7) except in the case where $\alpha, \beta \in \Lambda_{c}^{\text {re }}$ and $\operatorname{Supp}_{\Xi}(\alpha, \beta)$ is component-full. Lemma 3.4 says that $U^{s c s}=s U^{c}$. Furthermore, Lemma 3.6 says that the simple roots of $\Upsilon^{s c s}$ are obtained from the simple roots of $\Upsilon^{c}$ by the action of $s$. Thus $s$ also takes $\operatorname{Supp}_{\Xi}(\alpha)$ and $\operatorname{Supp}_{\Xi}(\beta)$ (with respect to $c$ ) to $\operatorname{Supp}_{\Xi}(s \alpha)$ and $\operatorname{Supp}_{\Xi}(s \beta)$ (with respect to $s c s)$. Therefore $\operatorname{adj}_{s \alpha}(s \beta)$, with respect to $s c s$, equals $\operatorname{adj}_{\alpha}(\beta)$, with respect to $c$.

We have verified (4.7) and now (4.8) follows by repeated applications of (4.7). It remains only to verify the uniqueness statements in Theorems 4.5 and 4.6. Together, Properties (4.5) and (4.6) specify the compatibility degree for all pairs of roots in $\Lambda_{c}$. By Proposition 3.12, we see that Properties (4.3), (4.4), and (4.8) completely specify the compatibility degree on all other pairs of roots, so we have proved the uniqueness in Theorem 4.6. But if a function on pairs of roots and Coxeter elements satisfies 
(4.7), then it satisfies (4.8) for each Coxeter element $c$, and thus it is uniquely determined by Properties (4.3) and (4.4) for each $c$. That is the uniqueness assertion of Theorem 4.5.

We now gather some properties of the compatibility degree.

The usual notion of compatibility degree for $\Phi$ of finite type, defined in [18, 26], is the unique function satisfying (4.3) and (4.8). See also [29, 43, 42]. (We have followed Ceballos and Pilaud [10, Remark 2.10] in modifying the usual notion by taking $(\alpha \| \alpha)_{c}=-1$ rather than 0 for $\alpha \in \Phi_{c}$.)

Thus the following is an immediate corollary of Theorem 4.6.

Corollary 4.9. If $\Phi$ is of finite type, then $(\alpha \| \beta)_{c}$ agrees with the usual compatibility degree on almost positive roots, modified to set $(\alpha \| \alpha)_{c}=-1$. Thus, the usual compatibility degree is $(\alpha \| \beta)_{c}=\max \left[(\alpha \rightarrow \beta)_{c},(\alpha \leftarrow \beta)_{c}\right]$.

Proposition 4.10. For all $\alpha, \beta \in \Phi_{c}$ we have $(\alpha \| \beta)_{c}=(\alpha \| \beta)_{c^{-1}}$.

Proof. We can write $(\alpha \| \beta)_{c^{-1}}$ because $\Phi_{c}=\Phi_{c^{-1}}$. (See Proposition 3.2.) Replacing $c$ with $c^{-1}$ swaps $(\alpha \rightarrow \beta)_{c}$ with $(\alpha \leftarrow \beta)_{c}$. Since $\Lambda_{c}^{\mathrm{re}}=\Lambda_{c^{-1}}^{\mathrm{re}}$, tube supports and the quantity $\operatorname{adj}_{\alpha}(\beta)$ are the same with respect to $c$ and $c^{-1}$.

In the following proposition, the quantity $\left(\alpha^{\vee} \| \beta^{\vee}\right)_{c}$ means the compatibility degree of $\alpha^{\vee}$ and $\beta^{\vee}$ in the root system $\Phi^{\vee}$. Since $\Phi$ and $\Phi^{\vee}$ define the same Weyl group, it makes sense to consider the same $c$ in both contexts. Furthermore, it is apparent (for example by Proposition 3.13) that $\Phi_{c}^{\vee}=\left\{\beta^{\vee}: \beta \in \Phi_{c}\right\}$.

Proposition 4.11. If $\alpha, \beta \in \Phi_{c}$, then $(\alpha \| \beta)_{c}=\left(\beta^{\vee} \| \alpha^{\vee}\right)_{c}$ except possibly when $\alpha, \beta \in$ $\Lambda_{c}^{\mathrm{re}}$ and $\operatorname{Supp}_{\Xi}(\alpha, \beta)$ is component-full, in which case $\left\{(\alpha \| \beta)_{c},\left(\beta^{\vee} \| \alpha^{\vee}\right)_{c}\right\} \subseteq\{1,2\}$.

Proof. Except when $\alpha, \beta \in \Lambda_{c}^{\text {re }}$ and $\operatorname{Supp}_{\Xi}(\alpha, \beta)$ is component-full, this is immediate from the definitions of $(\alpha \rightarrow \beta)_{c}$ and $(\alpha \leftarrow \beta)_{c}$. When $\alpha, \beta \in \Lambda_{c}^{\mathrm{re}}$ and $\operatorname{Supp}_{\Xi}(\alpha, \beta)$ is component-full, $\operatorname{Supp}_{\Xi}\left(\beta^{\vee}, \alpha^{\vee}\right)$ is also component-full. However, it is possible that $\operatorname{adj}_{\alpha}(\beta)=1$ while $\operatorname{adj}_{\beta^{\vee}}\left(\alpha^{\vee}\right)=2$ or vice-versa.

We now describe how the compatibility degree changes under rescaling.

Proposition 4.12. Suppose $\Phi$ and $\Phi^{\prime}$ are related by the rescaling $\beta \mapsto \beta^{\prime}=\lambda_{\beta} \beta$. Fix a Coxeter element $c$ of their common Weyl group. The rescaling map restricts to a bijection from $\Phi_{c}$ to $\Phi_{c}^{\prime}$. For $\alpha, \beta \in \Phi_{c}$, the compatibility degree $\left(\alpha^{\prime} \| \beta^{\prime}\right)_{c}$, computed in $\Phi^{\prime}$ equals $f \cdot(\alpha \| \beta)_{c}$, where $(\alpha \| \beta)_{c}$ is the compatibility degree computed in $\Phi$ and $f$ is some positive scalar. The scalar $f$ is $\frac{\lambda_{\beta}}{\lambda_{\alpha}}$, except possibly when $\alpha=\delta$.

Proof. Proposition 3.13 implies that $\beta \mapsto \beta^{\prime}$ restricts to a bijection from $\Phi_{c}$ to $\Phi_{c}^{\prime}$. Scaling roots does not affect membership in $U^{c}$. If $\alpha$ and $\beta$ are in $\Upsilon^{c}$ but in different components or if they are in the same component and have nested tube support, then $(\alpha \| \beta)_{c}$ and $\left(\alpha^{\prime} \| \beta^{\prime}\right)_{c}$ are both zero. Now suppose $\alpha$ and $\beta$ are in the same component of $\Upsilon^{c}$ but do not have nested tube supports. The two compatibility degrees are $\operatorname{adj}_{\alpha^{\prime}}\left(\beta^{\prime}\right)$ and $\operatorname{adj}_{\alpha}(\beta)$. Rescaling does not affect tube supports, so $\operatorname{adj}_{\alpha^{\prime}}\left(\beta^{\prime}\right)=\operatorname{adj}_{\alpha}(\beta)$. Since $\alpha$ and $\beta$ are of the same length and $\alpha^{\prime}$ and $\beta^{\prime}$ are of the same length, we have $\frac{\lambda_{\beta}}{\lambda_{\alpha}}=1$, and we are done in the case where $\alpha, \beta \in \Lambda_{c}^{\mathrm{re}}$.

We next show that $(\alpha \rightarrow \beta)_{c}$ and $(\alpha \leftarrow \beta)_{c}$ change as desired. When $\alpha$ and $\beta$ are positive, by Lemma 4.7 and the fact that $E_{c}$ is bilinear, this amounts to showing 
that $E_{c}$ is the same bilinear form whether it is defined in terms of $\Phi$ or $\Phi^{\prime}$. The real co-roots in $\Phi$ and $\Phi^{\prime}$ are related by

$$
\left(\beta^{\prime}\right)^{\vee}=\frac{2}{K\left(\beta^{\prime}, \beta^{\prime}\right)} \beta^{\prime}=\frac{2 \lambda_{\beta}}{\left(\lambda_{\beta}\right)^{2} K(\beta, \beta)} \beta=\frac{1}{\lambda_{\beta}} \beta^{\vee} .
$$

Using the definition (2.1) in terms of $\Phi$, we calculate $E_{c}\left(\left(\alpha_{i}^{\prime}\right)^{\vee}, \alpha_{j}^{\prime}\right)$ on simple roots/co-roots of $\Phi^{\prime}$ to be

$$
\frac{\lambda_{j}}{\lambda_{i}} E_{c}\left(\alpha_{i}^{\vee}, \alpha_{j}\right)= \begin{cases}\frac{\lambda_{j}}{\lambda_{i}} K\left(\alpha_{i}^{\vee}, \alpha_{j}\right)=K\left(\left(\alpha_{i}^{\prime}\right)^{\vee}, \alpha_{j}^{\prime}\right) & \text { if } i>j, \\ 1 & \text { if } i=j, \\ 0 & \text { if } i<j,\end{cases}
$$

where $\lambda_{i}$ is shorthand for $\lambda_{\alpha_{i}}$. This agrees with the definition of $E_{c}\left(\left(\alpha_{i}^{\prime}\right)^{\vee}, \alpha_{j}^{\prime}\right)$ in terms of $\Phi^{\prime}$. In light of (4.9), we see that $\left(\alpha^{\prime} \| \beta^{\prime}\right)_{c}=\frac{\lambda_{\beta}}{\lambda_{\alpha}}(\alpha \| \beta)_{c}$, except possibly when $\alpha=\delta$. Furthermore, when $\alpha=\delta$, (2.3) implies that $\left(\alpha^{\prime} \| \beta^{\prime}\right)_{c}=f \cdot(\alpha \| \beta)_{c}$ for some $f>0$, but there is no guarantee that $f=\frac{\lambda_{\beta}}{\lambda_{\alpha}}$.

If $\alpha=-\alpha_{j}$ for some $j$, then (4.3) says that $(\alpha \| \beta)_{c}=\left[\beta: \alpha_{j}\right]$ and $\left(\alpha^{\prime} \| \beta^{\prime}\right)_{c}=$ $\left[\beta^{\prime}: \alpha_{j}^{\prime}\right]$. The latter is equal to $\frac{\lambda_{\beta}}{\lambda_{\alpha}}\left[\beta: \alpha_{j}\right]$. If $\beta=-\alpha_{k}$, then (4.4) says that $(\alpha \| \beta)_{c}=\left[\alpha^{\vee}: \alpha_{k}^{\vee}\right]$ and $\left(\alpha^{\prime} \| \beta^{\prime}\right)_{c}=\left[\left(\alpha^{\prime}\right)^{\vee}:\left(\alpha_{k}^{\prime}\right)^{\vee}\right]$. If $\alpha$ is real, then (4.9) implies that $\left[\left(\alpha^{\prime}\right)^{\vee}:\left(\alpha_{k}^{\prime}\right)^{\vee}\right]=\frac{\lambda_{\beta}}{\lambda_{\alpha}}\left[\alpha^{\vee}: \alpha_{k}^{\vee}\right]$. If $\alpha=\delta$, then we use (2.3) again to conclude that $\left(\alpha^{\prime} \| \beta^{\prime}\right)_{c}=f \cdot(\alpha \| \beta)_{c}$ for some positive $f$.

Proposition 4.13. For $\alpha, \beta \in \Phi_{c}$, the quantities $(\beta \| \alpha)_{c}$ and $(\alpha \| \beta)_{c}$ are related by a positive scaling depending on $\alpha$ and $\beta$. When $\alpha, \beta \in \Phi_{c}^{\mathrm{re}}$, except possibly when $\alpha, \beta \in \Lambda_{c}^{\mathrm{re}}$ and $\operatorname{Supp}_{\Xi}(\alpha, \beta)$ is component-full,

$$
(\beta \| \alpha)_{c}=\frac{K(\alpha, \alpha)}{K(\beta, \beta)}(\alpha \| \beta)_{c} .
$$

Proof. Combine Proposition 4.11 with Proposition 4.12.

Proposition 4.14. For distinct roots $\alpha \neq \beta$ in $\Phi_{c}^{\mathrm{re}}$, we have $(\alpha \| \beta)_{c}>-1$.

Proof. This follows immediately from Theorem 4.6 and Proposition 3.13.

Proposition 4.15. Let $\Phi^{\prime}$ be a parabolic root subsystem of $\Phi$ and let $c^{\prime}$ be the restriction of $c$ to the corresponding parabolic subgroup $W^{\prime}$. Then, for any $\alpha, \beta \in \Phi_{c^{\prime}}^{\prime}$, we have $(\alpha \| \beta)_{c}=(\alpha \| \beta)_{c^{\prime}}$, where the latter compatibility degree is computed in $\Phi^{\prime}$.

Proof. Proposition 3.15 says that $\Phi_{c^{\prime}}^{\prime}=\Phi_{c} \cap \Phi^{\prime}$. If $\alpha$ and $\beta$ are in $\Lambda_{c}^{\text {re }}$ and $\operatorname{Supp}_{\Xi}(\alpha, \beta)$ is component-full, then $\alpha+\beta-\delta$ has nonnegative simple root coordinates. Since $\delta$ already has strictly positive simple root coordinates, we see that $\alpha$ and $\beta$ are not both contained in the same proper parabolic root subsystem, so this case is impossible. We thus restrict our attention to the case where $(\alpha \| \beta)_{c}=\max \left[(\alpha \rightarrow \beta)_{c},(\alpha \leftarrow \beta)_{c}\right]$. By the definition of $(\alpha \rightarrow \beta)_{c}$ and $(\alpha \leftarrow \beta)_{c}$, this maximum is $\max \left[(\alpha \rightarrow \beta)_{c^{\prime}},(\alpha \leftarrow \beta)_{c^{\prime}}\right]$ which is $(\alpha \| \beta)_{c^{\prime}}$ because $\Phi^{\prime}$ is finite.

Remark 4.16. The finite cases of several propositions in this section are known (see for example [26, Section 3]) but can also be proved by the same arguments given here for the affine case. 


\section{COMpatibility AND Clusters}

Definition 5.1. Distinct roots $\alpha$ and $\beta$ in $\Phi_{c}$ are c-compatible if and only if $(\alpha \| \beta)_{c}=0$. Proposition 4.13 implies that $c$-compatibility is a symmetric relation.

Remark 5.2. Definition 5.1 applies only to pairs of distinct roots. If we drop the requirement of distinctness, then by (4.6) and Proposition 4.14, the only root that is "c-compatible with itself" is $\delta$. We don't know whether the fact that $(\delta \| \delta)_{c}=0$ is significant, but we also see no reason to declare by fiat that $(\delta \| \delta)_{c}$ should be -1 .

Definition 5.3. The c-cluster complex is the abstract simplicial whose vertex set is $\Phi_{c}$ and whose faces are the sets in which any two distinct roots are $c$-compatible. Maximal faces in the $c$-cluster complex are called c-clusters. An imaginary $c$ cluster is a cluster containing $\delta$ and real c-cluster is a cluster not containing $\delta$.

Theorems 1.1 and 1.2, which we will prove in Section 9, state that the $c$-cluster complex (restricted to real $c$-clusters) is the cluster complex of the corresponding cluster algebra, and more specifically that real $c$-clusters are precisely the denominator vectors of clusters of cluster variables. It is currently less clear what imaginary $c$-clusters mean in the context of cluster algebras, but as stated in the introduction, we expect that $\delta$ is the denominator vector of an important element of the cluster algebra. An example of imaginary c-clusters can be seen in Example 6.5: There are two imaginary $c$-clusters, each composed of $\delta$ (pictured at infinity in Fig. 6.1) and one of the two roots in $\Lambda_{c}^{\text {re }}$ (pictured in cyan in the figure).

We now establish the essential properties of compatibility and clusters. The finite type versions of these properties can be found in $[18,26]$ or can be proved using the arguments given here.

The following proposition is immediate from the characterization of compatibility degree in Theorems 4.5 and 4.6 and Proposition 4.15.

Proposition 5.4. For any root system $\Phi$ of affine type and any Coxeter element $c$,

(1) $\tau_{c}$ acts as a permutation of the set of c-clusters.

(2) If $s$ is an initial or final reflection in $c$ then $\sigma_{s}$ acts as a bijection from the set of c-clusters in $\Phi_{c}$ to the set of scs-clusters in $\Phi_{\text {scs }}$.

(3) For each $i \in\{1, \ldots n\}$, write $\Phi^{\prime}$ for the parabolic root subsystem whose simple roots are $\Pi \backslash\left\{\alpha_{i}\right\}$ and write $c^{\prime}$ for the restriction of $c$ to the corresponding parabolic subgroup of $W$. Then $C \mapsto C \backslash\left\{-\alpha_{i}\right\}$ is a bijection from the set of c-clusters in $\Phi_{c}$ containing $-\alpha_{i}$ to the set of $c^{\prime}$-clusters in $\Phi_{c^{\prime}}^{\prime}$.

The following theorem reconciles Definition 5.3 with another reasonable way one might have defined "real $c$-clusters."

Theorem 5.5. A set $C \subseteq \Phi_{c}$ is a real c-cluster (a c-cluster not containing $\delta$ ) if and only if it is a maximal set of pairwise c-compatible roots in $\Phi_{c}^{\mathrm{re}}$.

A real $c$-cluster is certainly a maximal set of pairwise $c$-compatible roots in $\Phi_{c}^{\mathrm{re}}$, but Theorem 5.5 is needed because it is conceivable that a maximal set $C$ of pairwise $c$-compatible roots in $\Phi_{c}^{\mathrm{re}}$ can fail to be a $c$-cluster. This would happen precisely if every root in $C$ were $c$-compatible with $\delta$. To rule out this possibility, we need several preliminary results, one of which requires, for the first time in the paper, case-by-case checking using the classification of affine root systems.

Proposition 5.6. A root $\alpha \in \Phi_{c}^{\mathrm{re}}$ is c-compatible with $\delta$ if and only if $\alpha \in \Lambda_{c}^{\mathrm{re}}$. 
Proof. By (4.6), roots in $\Lambda_{c}^{\text {re }}$ are $c$-compatible with $\delta$. Since $\operatorname{Supp}_{\Pi}(\delta)=\Pi,(4.3)$ implies that $\left(-\alpha_{i} \| \delta\right)_{c} \neq 0$ for all $i$. Since $\tau_{c}$ fixes $\delta$, (4.8) completes the proof.

Definition 5.7. For any affine root system $\Phi$, we define $M_{\Phi}$ to be the maximal number of steps needed to go from any Coxeter element to a conjugate Coxeter element by a sequence of conjugations by initial simple reflections plus $n$ times the least common multiple of the ranks of the components of $\Upsilon^{c}$.

The precise quantity $M_{\Phi}$ is of no great theoretical importance, but appears as a convenient bound in several lemmas where the point is that some bound exists.

Lemma 5.8. Given an affine root system $\Phi$, a Coxeter element $c$ and a collection $R$ of real roots in $\Lambda_{c}^{\mathrm{re}}$ such that $\operatorname{Supp}_{\Xi}(R)$ is not component-full, there exists a sequence $a_{1}, \ldots, a_{\ell}$ of elements of $S$ with $\ell \leq M_{\Phi}$ such that

(i) $a_{i}$ is initial in the Coxeter element $a_{i-1} \cdots a_{1} c a_{1} \cdots a_{i-1}$ for all $i=1 \ldots \ell$, and

(ii) $\operatorname{Supp}_{\Pi}\left(a_{\ell} \cdots a_{1} R\right)$ is not full.

Proof. The conclusion of the lemma is not affected by rescaling the root system, so we may as well take $\Phi$ to be a standard affine root system. By an approporate choice of the initial letters of the sequence (making source-sink moves to change the Coxeter element), we may as well assume that $c$ as in Table 2.1. We will show that for such $c$ there exists an integer $m$ such that $\operatorname{Supp}_{\Pi}\left(c^{m}(R)\right)$ is not full. From there, one can complete the desired sequence, because the $c$-orbit of $R$ is no larger than the least common multiple of the ranks of the components of $\Upsilon^{c}$.

Indexing the components of $\Upsilon^{c}$ by indices $i$, we write $\beta_{\text {aff }}^{i}$ for the root $\delta-\sum \beta$, where the sum is over those simple roots of the $i^{\text {th }}$ component that are also in $\Phi_{\text {fin }}$. Thus $\beta_{\mathrm{aff}}^{i}$ is the unique simple root in the $i^{\text {th }}$ component that is not in $\Phi_{\text {fin }}$. In each case, because $c$ cyclically permutes the simple roots of each component of $\Upsilon^{c}$ and since $\operatorname{Supp}_{\Xi}(R)$ is not component-full, by applying $c$ some number of times, we may as well assume that $\beta_{\text {aff }}^{1}$ is not in $\operatorname{Supp}_{\Xi}(R)$. (That is, the intersection of $R$ with the first component contains only roots in $\Phi_{\text {fin. }}$.) We now finish the proof in each case. In each case, the simple generators of $W$ are numbered as in Table 2.1 and $c$ is $s_{1} \cdots s_{n}$. We index components of $\Upsilon^{c}$ compatibly with the numbering of the roots $\beta_{j}$ in Table 2.1: The first component contains $\beta_{1}$, the second component contains $\beta_{j}$ for the smallest $j$ such that $\beta_{j}$ is not in the first component, etc.

In the proof, we use explicit simple-root coordinates of $\delta$ in types $A, B, D$, and $E_{6}$. These can be found, for example, in [24, Table Aff 1].

Case $A_{n-1}^{(1)}:$ If $k \in\{1, n-1\}$, then there is nothing to prove. Otherwise, if $\beta_{\text {aff }}^{2}$ is not in $\operatorname{Supp}_{\Xi}(R)$, then $\alpha_{n} \notin \operatorname{Supp}_{\Pi}(R)$. If $\beta_{\text {aff }}^{2} \in \operatorname{Supp}_{\Xi}(R)$, then since $\operatorname{Supp}_{\Xi}(R)$ is not component-full, there exists $j \in\{k, \ldots, n-2\}$ such that $\beta_{j} \notin \operatorname{Supp}_{\Xi}(R)$. In this case, since $\beta_{\mathrm{aff}}^{2}=\delta-\sum_{j=k}^{n-2} \beta_{j}=\alpha_{n}+\sum_{i=1}^{k} \alpha_{i}$, we see that $\alpha_{j+1} \notin \operatorname{Supp}_{\Pi}(R)$.

Case $B_{n-1}^{(1)}$ : If $\beta_{\text {aff }}^{2}$ is not in $\operatorname{Supp}_{\Xi}(R)$, then $\alpha_{n} \notin \operatorname{Supp}_{\Pi}(R)$. If $\beta_{\text {aff }}^{2} \in \operatorname{Supp}_{\Xi}(R)$, then since $\operatorname{Supp}_{\Xi}(R)$ is not component-full, $\beta_{n-2}$ is not in $\operatorname{Supp}_{\Xi}(R)$. Thus since $\beta_{\mathrm{aff}}^{2}=\delta-\beta_{n-2}=\alpha_{n}+\sum_{i=1}^{n-2} \alpha_{i}$, we see that $\alpha_{n-1} \notin \operatorname{Supp}_{\Pi}(R)$.

Case $C_{n-1}^{(1)}$ : In this case, $\Upsilon^{c}$ has only one component. Since $\beta_{\text {aff }}^{1} \notin \operatorname{Supp}_{\Xi}(R)$, we have $\operatorname{Supp}_{\Xi}(R) \subseteq \Phi_{\text {fin }}$ and thus $\alpha_{\text {aff }}=\alpha_{n} \notin \operatorname{Supp}_{\Pi}(R)$.

Case $D_{n-1}^{(1)}$ : There are four cases depending on whether $\beta_{\text {aff }}^{2}$ and $\beta_{\text {aff }}^{3}$ are in $\operatorname{Supp}_{\Xi}(R)$. If $\beta_{\text {aff }}^{2} \notin \operatorname{Supp}_{\Xi}(R)$ and $\beta_{\text {aff }}^{3} \notin \operatorname{Supp}_{\Xi}(R)$, then $\operatorname{Supp}_{\Xi}(R) \subseteq \Phi_{\text {fin }}$, so $\alpha_{\text {aff }}=\alpha_{n} \notin \operatorname{Supp}_{\Pi}(R)$. Now suppose $\beta_{\text {aff }}^{2} \in \operatorname{Supp}_{\Xi}(R)$, so that $\beta_{n-3} \notin \operatorname{Supp}_{\Xi}(R)$. 
If furthermore $\beta_{\text {aff }}^{3} \notin \operatorname{Supp}_{\Xi}(R)$, then since $\beta_{\text {aff }}^{2}=\delta-\beta_{n-3}=\alpha_{n}+\sum_{i=2}^{n-2} \alpha_{i}$, we have $\alpha_{1} \notin \operatorname{Supp}_{\Pi}(R)$. If, on the other hand, $\beta_{\text {aff }}^{3} \in \operatorname{Supp}_{\Xi}(R)$, then $\beta_{n-2} \notin \operatorname{Supp}_{\Xi}(R)$. Since $\beta_{\text {aff }}^{3}=\delta-\beta_{n-2}=\alpha_{1}+\alpha_{n}+\sum_{i=3}^{n-2} \alpha_{i}$, we have $\alpha_{n-1} \notin \operatorname{Supp}_{\Pi}(R)$. Finally, if $\beta_{\text {aff }}^{2} \notin \operatorname{Supp}_{\Xi}(R)$ and $\beta_{\text {aff }}^{3} \in \operatorname{Supp}_{\Xi}(R)$, then $\beta_{n-2} \notin \operatorname{Supp}_{\Xi}(R)$, so $\alpha_{2} \notin \operatorname{Supp}_{\Pi}(R)$.

Case $E_{6}^{(1)}:$ Since there are 3 simple roots in component 1 of $\Upsilon^{c}$, permuted cyclically by $c$ and 2 simple roots in component 3 , permuted cyclically by $c$, and since $\operatorname{gcd}(2,3)=1$, we can (by applying $c$ repeatedly) assume that neither $\beta_{\text {aff }}^{1}$ nor $\beta_{\text {aff }}^{3}$ is in $\operatorname{Supp}_{\Xi}(R)$. If furthermore $\beta_{\text {aff }}^{2} \notin \operatorname{Supp}_{\Xi}(R)$, then $\alpha_{\text {aff }}=\alpha_{7} \notin \operatorname{Supp}_{\Pi}(R)$. If $\beta_{\text {aff }}^{2} \in \operatorname{Supp}_{\Xi}(R)$, then either $\beta_{3}$ or $\beta_{4}$ is not in $\operatorname{Supp}_{\Xi}(R)$. If $\beta_{4} \notin \operatorname{Supp}_{\Xi}(R)$, then since $\beta_{\text {aff }}^{2}=\delta-\left(\beta_{3}+\beta_{4}\right)=\sum_{i \neq 3} \alpha_{i}$, we see that $\alpha_{3} \notin \operatorname{Supp}_{\Pi}(R)$. If $\beta_{3} \notin \operatorname{Supp}_{\Xi}(R)$, then we consider the collection $c^{2} R$. None of the $\operatorname{roots} \beta_{2}, \beta_{\mathrm{aff}}^{2}$, and $\beta_{\mathrm{aff}}^{3}$ is in $\operatorname{Supp}_{\Xi}\left(c^{2} R\right)$. Since $\beta_{\text {aff }}^{1}=\delta-\left(\beta_{1}+\beta_{2}\right)=\sum_{i=2}^{7} \alpha_{i}$, we see that $\alpha_{1} \notin \operatorname{Supp}_{\Pi}(R)$.

Case $E_{7}^{(1)}$ : The ranks of the components of $\Upsilon^{c}$ are 4,3 , and 2 . Since $\operatorname{gcd}(4,3,2)=1$, we can apply $c$ until $\left\{\beta_{\text {aff }}^{i}: i=1,2,3\right\} \cap \operatorname{Supp}_{\Xi}(R)=\varnothing$. Then $\alpha_{\text {aff }}=\alpha_{8} \notin \operatorname{Supp}_{\Pi}(R)$. Case $E_{8}^{(1)}$ : We proceed as in Case $E_{7}^{(1)}$. The ranks are 5,3 , and 2.

Case $F_{4}^{(1)}$ : We proceed as in the previous two cases. The ranks are 3 and 2 . Case $G_{2}^{(1)}$ : In this case, $\Upsilon^{c}$ has only component, so we proceed as in Case $C_{n-1}^{(1)}$.

Proposition 5.9. For every affine root system $\Phi$, Coxeter element $c$ and collection $R$ of roots in $\Lambda_{c}^{\mathrm{re}}$ such that $\operatorname{Supp}_{\Xi}(R)$ is not component-full, there exists a root $\alpha \in \Phi_{c}^{\mathrm{re}} \backslash \Lambda_{c}^{\mathrm{re}}$ that is c-compatible with every root in $R$.

Proof. Let $s_{i_{1}}, \ldots, s_{i_{k}}$ be a sequence as in Lemma 5.8 and let $\alpha_{j}$ be a simple root not in $\operatorname{Supp}_{\Pi}\left(s_{i_{k}} \cdots s_{i_{1}} R\right)$. Each $\beta \in R$ is positive and a simple induction using Lemma 3.5 implies that $s_{i_{j}} \cdots s_{i_{1}}(\beta)$ is positive for $j=1, \ldots, k$. Thus $\sigma_{i_{k}} \cdots \sigma_{i_{1}}(\beta)=$ $s_{i_{k}} \cdots s_{i_{1}}(\beta)$. Now (4.3) implies that $-\alpha_{j}$ is $\left(s_{i_{m}} \cdots s_{i_{1}} c s_{i_{1}} \cdots s_{i_{m}}\right)$-compatible with $\sigma_{i_{k}} \cdots \sigma_{i_{1}} \beta$. By Lemma 3.11 and (4.7), the desired root is $\alpha=\sigma_{i_{1}} \cdots \sigma_{i_{k}}\left(-\alpha_{j}\right)$.

Lemma 5.10. If $R$ is a set of pairwise c-compatible roots in $\Lambda_{c}^{\mathrm{re}}$, then $\operatorname{Supp}_{\Xi}(R)$ is not component-full.

Proof. We need to show that there is a simple root of each component of $\Upsilon^{c}$ that is not in $\operatorname{Supp}_{\Xi}(R)$. Fix a component $\Upsilon^{\prime}$ of $\Upsilon^{c}$ and, if $\operatorname{Supp}_{\Xi}(R)$ includes any simple roots in $\Upsilon^{\prime}$, choose $\beta \in R$ in $\Upsilon^{\prime}$ so that $\operatorname{Supp}_{\Xi}(\beta)$ is maximal, under containment, among tube supports of roots in $R$. By Lemma $3.10, \operatorname{Supp}_{\Xi}(\beta)$ is not componentfull. Thus $\operatorname{Supp}_{\Xi}(\beta)$ is properly contained in the set of simple roots for $\Upsilon^{\prime}$. By (4.5) and since $\beta$ was chosen to have maximal tube support, any root $\beta^{\prime} \in R \backslash\{\beta\}$ has either $\operatorname{Supp}_{\Xi}\left(\beta^{\prime}\right) \mp \operatorname{Supp}_{\Xi}(\beta)$ or $\operatorname{adj}_{\beta}\left(\beta^{\prime}\right)=0$. In particular, the simple root(s) of $\Upsilon^{\prime}$ that are adjacent to $\beta$ are not in $\operatorname{Supp}_{\Xi}(R)$, and the lemma is proved.

Proof of Theorem 5.5. Let $R$ be a set of pairwise $c$-compatible roots in $\Phi_{c}^{\text {re }}$, all $c$-compatible with $\delta$. Lemma 5.10 says that $R$ satisfies the conditions on $R$ in Proposition 5.9, which guarantees the existence a root $\alpha$ in the $\tau_{c}$-orbit of a negative simple root that is $c$-compatible with every root in $R$. In particular, $R$ is not a maximal set of pairwise $c$-compatible roots.

We next discuss compatibility among positive roots in $\Lambda_{c}^{\text {re }}$.

Definition 5.11. Two roots $\alpha, \beta \in \Lambda_{c}^{\text {re }}$ are nested if $\operatorname{Supp}_{\Xi}(\alpha) \subseteq \operatorname{Supp}_{\Xi}(\beta)$ or $\operatorname{Supp}_{\Xi}(\beta) \subseteq \operatorname{Supp}_{\Xi}(\alpha)$. They are spaced if $\operatorname{Supp}_{\Xi}\left(c^{-1} \alpha\right) \cup \operatorname{Supp}_{\Xi}(\alpha) \cup \operatorname{Supp}_{\Xi}(c \alpha)$ is disjoint from $\operatorname{Supp}_{\Xi}(\beta)$. 
Each notion in Definition 5.11 is symmetric. The roots $\alpha$ and $\beta$ are spaced if and only if $\operatorname{Supp}_{\Xi}(\alpha)$ is disjoint from $\operatorname{Supp}_{\Xi}(\beta)$ and $\operatorname{adj}_{\alpha}(\beta)=0$. In particular, roots from distinct components of $\Upsilon^{c}$ are spaced. The next proposition follows immediately from (4.5).

Proposition 5.12. Two distinct roots $\alpha$ and $\beta$ in $\Lambda_{c}^{\mathrm{re}}$ are c-compatible if and only if they are nested or spaced.

Proposition 5.12 allows us to connect the notion of compatibility among roots in $\Lambda_{c}^{\text {re }}$ to some well-known combinatorics. Supports of roots in $\Lambda_{c}^{\text {re }}$ correspond to tubes, in the sense of graph associahedra [9, Definition 2.2], in the Dynkin diagram of $\Upsilon^{c}$. Compatibility of these roots corresponds to compatibility of tubes in the same sense. Thus the restriction of the $c$-cluster complex to roots in $\Lambda_{c}^{\text {re }}$ is isomorphic to the boundary complex of the simplicial graph associahedron for the Dynkin diagram of $\Upsilon^{c}$, the polytope dual to the (simple) graph associahedron of [9, Definition 2.4]. Each component of the Dynkin diagram of $\Upsilon^{c}$ is a cycle or consists of two vertices connected by an edge. The simplicial graph associahedron of a $k$-cycle is a $(k-1)$-dimensional simplicial cyclohedron (so its boundary complex is a $(k-2)$-dimensional simplicial complex). The simplicial graph associahedron associated to two vertices connected by an edge is a line segment (so its boundary complex is two isolated vertices, i.e. a 0-dimensional sphere), and we extend terminology slightly to call this a 1-dimensional simplicial cyclohedron. We summarize in the following proposition.

Proposition 5.13. In affine type, the restriction of the c-cluster complex to roots in $\Lambda_{c}^{\mathrm{re}}$ is $(n-3)$-dimensional and isomorphic to a join of boundary complexes of simplicial cyclohedra. There is a $(k-1)$-dimensional cyclohedron (and thus a $(k-2)$ dimensional boundary complex) for each rank-k component of $\Upsilon^{c}$.

We conclude the section by cataloging the crucial facts about real and imaginary $c$-clusters. The finite-type versions of these facts are in [18, 26], or can be proved as outlined here for affine type. We use the notation $Q$ for the root lattice of $\Phi$ and $Q_{c}$ for the sublattice $Q \cap U^{c}$, which should be thought of as the root lattice of $\Upsilon^{c}$. In the following proposition, we interpret indices in the expressions $\sigma_{k} \cdots \sigma_{1}$ and $s_{k} \cdots s_{1}$ modulo $n$ and, if $k<0$ we interpret the expression $\sigma_{k} \cdots \sigma_{1}$ to mean $\sigma_{k+1} \sigma_{k+2} \cdots \sigma_{-1} \sigma_{0}$ and similarly for $s_{k} \cdots s_{1}$.

Proposition 5.14. Let $\Phi$ be a root system of affine type, let $c=s_{1} \cdots s_{n}$ be a Coxeter element, and let $C$ be a c-cluster. If $C$ is a real c-cluster, then

(1) $|C|=n$,

(2) $C$ is a $\mathbb{Z}$-basis for the root lattice $Q$ of $\Phi$,

(3) $C$ contains at least 2 roots in the $\tau_{c}$-orbits of negative simple roots, and

(4) There exists an integer $k$ and an index $i \in\{1, \ldots, n\}$ such that

$$
\sigma_{k} \cdots \sigma_{1}(C)=s_{k} \cdots s_{1}(C)=\left\{-\alpha_{i}\right\} \cup C^{\prime}
$$

for a $c^{\prime}$-cluster $C^{\prime}$ of roots in $\Phi_{c^{\prime}}^{\prime}$, where $\Phi^{\prime}$ is the parabolic root subsystem spanned by $\Pi \backslash\left\{\alpha_{i}\right\}$ and $c^{\prime}$ is the restriction of $s_{k} \cdots s_{1} c s_{1} \cdots s_{k}$ to the corresponding parabolic subgroup of $W$.

If $C$ is an imaginary c-cluster, then

(5) $|C|=n-1$,

(6) $C$ is a $\mathbb{Z}$-basis for the lattice $Q_{c}$, 
(7) $C$ contains no roots in the $\tau_{c}$-orbits of negative simple roots, and

(8) $C \backslash\{\delta\}$ consists of roots in $\Lambda_{c}^{\mathrm{re}}$.

Proof. First, assume $C$ is an imaginary c-cluster. Proposition 5.6 implies (7) and (8). Thus Proposition 5.13 implies (5). Furthermore, one easily convinces oneself that for each $\operatorname{root} \beta$ in $C$, there exists a unique $\beta_{i} \in \operatorname{Supp}_{\Xi}(\beta)$ such that $\beta_{i}$ is not in the tube support of any root $\beta^{\prime} \in C$ with $\operatorname{Supp}_{\Xi}\left(\beta^{\prime}\right) \mp \operatorname{Supp}_{\Xi}(\beta)$. Thus the $\Xi^{c}$-coordinates of roots in $C$ form a unitriangular matrix with respect to a linear extension of the containment order on tube supports of roots in $C$. If $\Upsilon^{c}$ is irreducible, then $\Xi^{c}$ is a basis for $Q_{c}$, so it follows that $C$ is also a basis. In general, $C$ contains a basis for each component of $\Upsilon^{c}$, with these bases overlapping exactly in the root $\delta$, and (6) follows.

Now, assume $C$ is a real $c$-cluster. Theorem 5.5 and Proposition 5.6 together imply that there exists an integer $\ell$ such that $\tau_{c}^{\ell}(C)$ contains a negative simple root. That is, using the convention described before the statement of the proposition, $\sigma_{-n \ell} \cdots \sigma_{1}(C)$ contains a negative simple root. If $\ell \leq 0$, choose the smallest $k$ with $0 \leq k \leq-n \ell$ such that $\sigma_{k} \cdots \sigma_{1}(C)$ contains a negative simple root $-\alpha_{i}$. If $\ell>0$, then choose the largest $k$ with $-n \ell \leq k \leq 0$ such that $\sigma_{k} \cdots \sigma_{1}(C)$ contains a negative simple root $-\alpha_{i}$. Because of this choice of $k$, in either case, we know that each application of $\sigma_{j}$ in the expression $\sigma_{k} \cdots \sigma_{1}(C)$ acts as $s_{j}$ on $C$, and thus $\sigma_{k} \cdots \sigma_{1}(C)=$ $s_{k} \cdots s_{1}(C)$. Proposition 5.4(2) implies that $\sigma_{k} \cdots \sigma_{1}(C)$ is a $s_{k} \cdots s_{1} c s_{1} \cdots s_{k}$-cluster. Now (4) follows by Proposition 5.4(3), with $C^{\prime}=\left(\sigma_{k} \cdots \sigma_{1}(C)\right) \backslash\left\{-\alpha_{i}\right\}$.

In particular, $s_{k} \cdots s_{1}(C)=\left\{-\alpha_{i}\right\} \cup C^{\prime}$. Since necessarily $\Phi^{\prime}$ is of finite type, $C^{\prime}$ is a real $c^{\prime}$-cluster. By the finite version of this result (or by induction), $C^{\prime}$ is a $\mathbb{Z}$-basis for the root lattice of $\Phi^{\prime}$, and therefore $s_{k} \cdots s_{1}(C)$ is a $\mathbb{Z}$-basis for $Q$. Since elements of $W$ preserve the lattice, $C$ itself is also a $\mathbb{Z}$-basis for $Q$. This yields (2), and (1) follows immediately. By Proposition 5.13, the largest possible size of a set of pairwise $c$-compatible roots in $\Lambda_{c}^{\text {re }}$ is $n-2$, and (3) follows by Proposition 3.12(5).

\section{Cluster expansions And the Cluster fan}

In this section, we show that the $c$-compatibility relation defines a simplicial fan, or equivalently that each vector in $V$ has a unique $c$-cluster expansion. Finite-type versions of these results are in $[18,26,42]$.

Definition 6.1. Given a vector in $v \in V$, a c-cluster expansion of $v$ is an expression $v=\sum_{\alpha \in \Phi_{c}} m_{\alpha} \alpha$ where the $m_{\alpha}$ are nonnegative real numbers having $m_{\alpha} m_{\beta}=0$ whenever $\alpha$ and $\beta$ are distinct and not $c$-compatible. In light of Proposition 5.14, at most $n$ of the $m_{\alpha}$ can be nonzero. The support of a $c$-cluster expansion $v=\sum_{\alpha \in \Phi_{c}} m_{\alpha} \alpha$ is the set $\left\{\alpha \in \Phi_{c}: m_{\alpha} \neq 0\right\}$.

Theorem 6.2. For any root system $\Phi$ of affine type and any Coxeter element $c$, every vector in $V$ admits a unique c-cluster expansion.

We emphasize that a vector may have a $c$-cluster expansion is terms of a real $c$-cluster or an imaginary c-cluster (or both, if the support of the expansion is contained in the intersection of the two clusters).

Before proving Theorem 6.2, we rephrase it geometrically. A simplicial cone is the nonnegative linear span of a linearly independent set $X$ of vectors and a face of the cone is the nonnegative linear span of a subset of $X$. A simplicial fan is a collection of simplicial cones that is closed under passing to faces, and such that 
the intersection of any two cones in the collection is a face of each. A simplicial fan is complete if the union of its cones is the entire ambient vector space.

Definition 6.3. Given a set $C$ of pairwise $c$-compatible roots in $\Phi_{c}$, write Cone $(C)$ for the nonnegative linear span of $C$. Write $\operatorname{Fan}_{c}(\Phi)$ for the collection of cones Cone $(C)$ for all sets $C$ of pairwise $c$-compatible roots in $\Phi_{c}$. Write $\operatorname{Fan}_{c}^{\mathrm{re}}(\Phi)$ for the subfan of $\operatorname{Fan}_{c}(\Phi)$ consisting of cones spanned by real roots. We call $\operatorname{Fan}_{c}(\Phi)$ the affine cluster fan or affine generalized associahedron fan.

Proposition 5.14(2) and Proposition 5.14(6) imply that the cones in $\operatorname{Fan}_{c}(\Phi)$ are simplicial. The content of Theorem 6.2 is that every point in $V$ is in some cone of $\operatorname{Fan}_{c}(\Phi)$ and that any two cones of $\operatorname{Fan}_{c}(\Phi)$ intersect in such a way that, for any $v$ in their intersection, the $c$-cluster expansions of $v$ coming from the two cones agree. This is precisely the assertion that the two cones intersect along a mutual face. Thus Theorem 6.2 has the following rephrasing.

Theorem 6.4. For any root system $\Phi$ of affine type and any Coxeter element $c$, $\operatorname{Fan}_{c}(\Phi)$ is a complete, simplicial fan.

Example 6.5. An example of $\operatorname{Fan}_{c}(\Phi)$ is shown in Fig. 6.1, for $\Phi$ of type $D_{3}^{(2)}$ and $c=s_{1} s_{2} s_{3}$. (Compare [36, Example 1.3].) The intersection of $\operatorname{Fan}_{c}(\Phi)$ with a unit sphere is a collection of points, geodesic arcs, and spherical triangles. The figure shows that collection, stereographically projected to the plane. The cone spanned by $-\Pi$ appears as the smallest triangle on the vertical line of symmetry of the picture. The colored points are (the directions of) some of the roots in $\Phi_{c}$, with a different color for each $\tau_{c}$-orbit.

We now prepare to prove Theorem 6.2. Let $\Delta_{c}$ be the nonnegative linear span of the positive roots in $\Upsilon^{c}$. This is a closed polyhedral cone whose extreme rays are the simple roots $\Xi^{c}$ of $\Upsilon^{c}$. Thus the cross section of $\Delta_{c}$ is a product of simplices, with one $k$-vertex simplex for each rank-k component of $\Upsilon^{c}$. The imaginary root $\delta$ is in the relative interior of $\Delta_{c}$, which we thus call the imaginary cone. (This is not the same as the cone spanned by positive imaginary roots, which is the ray spanned by $\delta$. Typically $\Delta_{c}$ contains infinitely many positive real roots.) We write $\Delta_{c}$ for the relative interior of $\Delta_{c}$. As an immediate consequence of Lemma 3.6, we have the following proposition and corollary.

Proposition 6.6. If $s$ is initial or final in $c$, then $\Delta_{s c s}=s \Delta_{c}$.

Corollary 6.7. $c \Delta_{c}=\Delta_{c}$.

We separate out one case of Theorem 6.2 as the following proposition.

Proposition 6.8. Every vector $v \in \Delta_{c}$ has a c-cluster expansion. This expansion is the unique c-cluster expansion for $v$ that is supported on roots in $\Lambda_{c}$.

Proof. Such a vector $v$ can be written as a finite positive linear combination of simple roots of $\Upsilon^{c}$. The simple roots in each component of $\Upsilon^{c}$ sum to $\delta$. Thus we can write $v$ as a linear combination, with nonnegative coefficients, of $\delta$ and elements of $\Xi^{c}$, with at least one zero coefficient in each component of $\Upsilon^{c}$. This expression is unique because the linear spans of any two components of $\Upsilon^{c}$ only intersect in the line spanned by $\delta$. For the same reason, the proposition reduces to proving the following claim: A vector $v^{\prime}$ in the nonnegative linear span of a proper parabolic root subsystem of a component $\Upsilon^{\prime}$ of $\Upsilon^{c}$ has a unique cluster expansion supported 


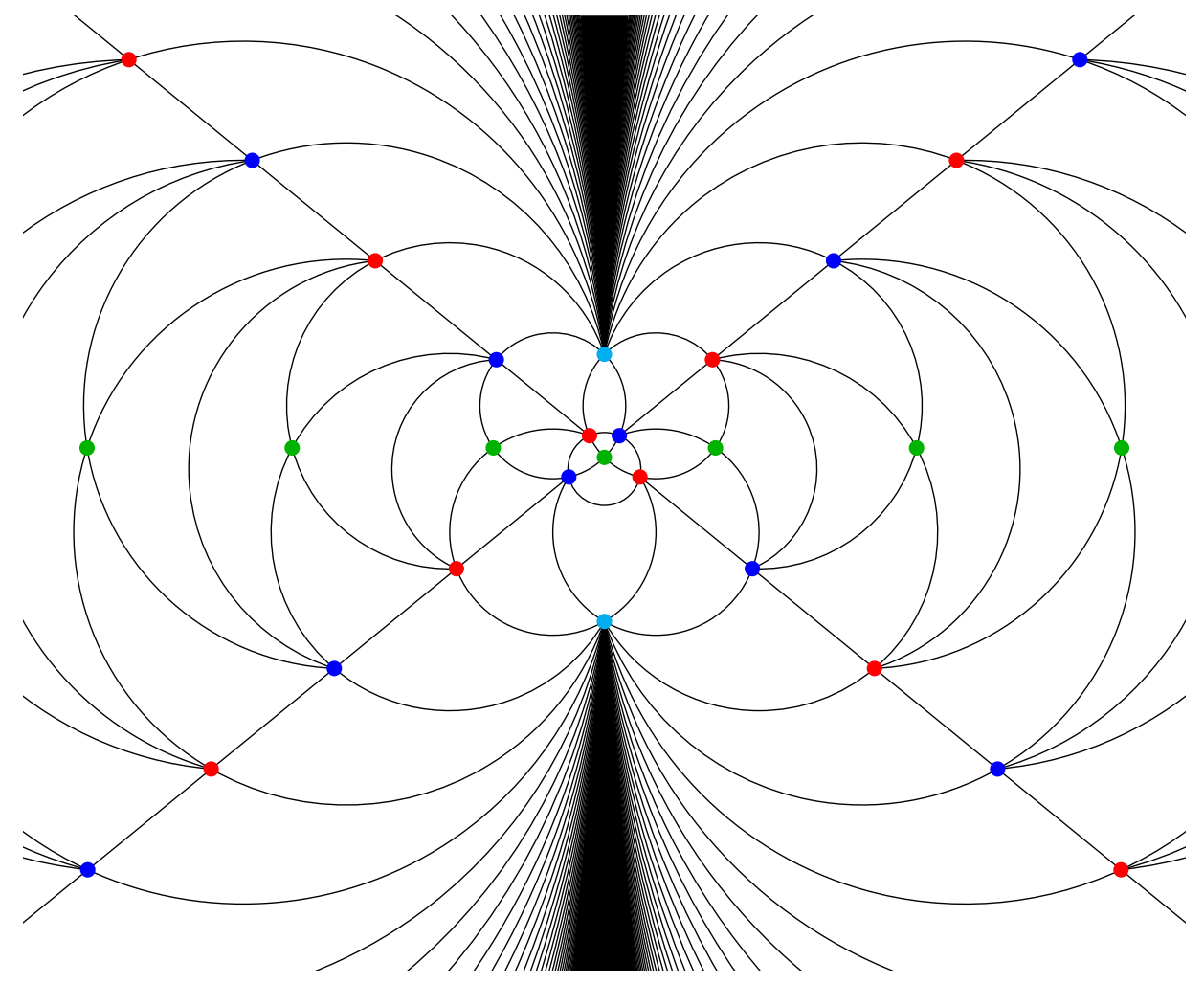

Figure 6.1. $\operatorname{Fan}_{c}(\Phi)$ for $\Phi$ and $c$ as in Example 6.5

on positive real roots in that component. (One may be tempted to directly deduce the claim from the finite case, in the parabolic subsystem. This does not work, because the notions of proper parabolics for $\Upsilon^{c}$ and $\Phi$ do not coincide.)

Given such a $v^{\prime}$, we can write $v^{\prime}=\sum_{\beta \in N^{\prime}} x_{\beta} \beta$ for a unique $N^{\prime} \mp \Xi^{c} \cap \Upsilon^{\prime}$ with $x_{\beta}>0$ for all $\beta \in N^{\prime}$. Let $x=\min \left\{x_{\beta}: \beta \in N^{\prime}\right\}$. By hypothesis, $N^{\prime}$ generates a root subsystem whose components are all of finite type $A$. Let $\beta \in N^{\prime}$ have $x_{\beta}=x$ and let $\alpha$ have maximal tube support among roots in this subsystem with $\beta \in \operatorname{Supp}_{\Xi}(\alpha)$ such that $v^{\prime \prime}=v^{\prime}-x \alpha$ is in the nonnegative linear span of $N^{\prime}$. Write $v^{\prime \prime}=\sum_{\beta \in N^{\prime \prime}} y_{\beta} \beta$ with $N^{\prime \prime} \mp N^{\prime}$ and $y_{\beta}>0$ for all $\beta \in N^{\prime \prime}$. By the minimality of $x$ and maximality of $\alpha, \alpha$ is either spaced or nested with all the roots in the subsystem generated by $N^{\prime \prime}$. The existence of a $c$-cluster expansion for $v^{\prime}$ then follows by induction on $\left|N^{\prime}\right|$.

Let $\ell$ be the minimum integer such that there exists a vector $v^{\prime}$ with $\left|N^{\prime}\right|=\ell$, having two distinct $c$-cluster expansions. By minimality of $\ell$, the set $N^{\prime}$ (as in the previous paragraph) induces a connected proper subgraph of the diagram of $\Upsilon^{\prime}$, and this subgraph is necessarily a path. This path is the tube support of the root $\alpha$. In light of Proposition 5.12, every c-cluster expansion of $x^{\prime}$ must include $\alpha$ with coefficient $x$. Therefore the vector $v^{\prime \prime}=v^{\prime}-x \alpha$ has two $c$-cluster expansions of length $\ell-1$, contradicting our assumption. We have proved the claim.

Besides proving part of Theorem 6.2, Proposition 6.8 is helpful in proving the following key lemma. 
Lemma 6.9. Given a nonzero $v \in V \backslash \check{\Delta}_{c}$, there exists a sequence of simple reflections $a_{1}, \ldots, a_{k}$ such that

(i) $a_{i}$ is initial or final in the Coxeter element $a_{i-1} \cdots a_{1} c a_{1} \cdots a_{i-1}$ for $i=1 \ldots k$, and

(ii) there is at least one nonpositive coefficient in the expansion of $a_{k} a_{k-1} \cdots a_{1}(v)$ in the basis of simple roots of $\Phi$.

The sequence can be chosen so that, in condition (i), either $a_{i}$ is initial for all $i$ or $a_{i}$ is final for all $i$. If $v \in U^{c} \backslash \triangle_{c}$, then the sequence can be chosen with $k \leq M_{\Phi}$.

Proof. First, suppose $v \in U^{c}$. For large enough positive $x \in \mathbb{R}$, the vector $v+x \delta$ is in $\Delta_{c}$, and thus by Proposition 6.8, the vector $v+x \delta$ has a cluster expansion consisting of roots in $\Lambda_{c}$. We can choose $x$ so that $\delta$ is not in the support of the expansion (or in other words, so that $v+x \delta$ is on the boundary of $\Delta_{c}$ ). We have $x \geq 0$, because by hypothesis $v \notin \stackrel{\Delta}{c}_{c}$. Write $\sum x_{i} \beta_{i}$ for the cluster expansion of $v+x \delta$, so that $v=-x \delta+\sum x_{i} \beta_{i}$. In light of Lemma 5.10, we can apply Lemma 5.8 to find a sequence $a_{1}, \ldots, a_{k}$ satisfying the first condition of the lemma (choosing "initial" for each $i$ ) and a simple root $\alpha_{j}$ with $\left[\sum x_{i} a_{k} a_{k-1} \cdots a_{1} \beta_{i}: \alpha_{j}\right]=0$. Since $\delta$ has full support in the basis of simple roots, we see that $\left[-x \delta+\sum x_{i} a_{k} a_{k-1} \cdots a_{1} \beta_{i}: \alpha_{j}\right] \leq 0$, and since $a_{k} a_{k-1} \cdots a_{1} \delta=\delta$, the inequality says $\left[a_{k} a_{k-1} \cdots a_{1} v: \alpha_{j}\right] \leq 0$.

Now suppose $v \notin U^{c}$ and write $v=a \gamma_{c}+b \delta+w$ with $w \in U^{c} \cap V_{\text {fin }}$ and $a \neq 0$. Proposition 2.10(3) says that $c$ has finite order on $U^{c}$. Thus there exists a positive integer $\ell$ such that $c^{\ell} w=w$. Since $c \gamma_{c}=\gamma_{c}+\delta$, we compute $c^{m \ell} v$ to be $a \gamma_{c}+(b+$ $m \ell a) \delta+w$ for any $m \in \mathbb{Z}$. We can choose $m$ so that $c^{m} v$ has at least one negative simple-root coordinate. If $m=0$, then we are done. If $m<0$, then the sequence $s_{-m n}, s_{-m n+1}, \ldots, s_{1}$, with indices interpreted modulo $n$, has the desired properties (choosing "initial" for each $i$ ). If $m>0$, then the sequence $s_{1}, s_{2}, \ldots, s_{m n}$, again with indices modulo $n$, is the desired sequence (with "final" for all $i$ ).

Proof of Theorem 6.2. A natural way to prove this theorem is to state it more broadly for finite and affine type together and argue by induction on rank. However, since when $\Phi$ is affine, every proper parabolic root subsystem of $\Phi$ is finite, and since the finite case is known ([18, Theorem 3.11] and [26, Proposition 3.6]), we appeal to the finite case rather than to induction.

Let $v$ be a vector in $V$. First, suppose $v$ is zero. Then $v$ has a cluster expansion with no terms. If some other cluster expansion exists, then some negative simple root $-\alpha_{i}$ must occur in the expansion with positive coefficient. Therefore, by (4.3), the other roots in the support of the second cluster expansion are in the complementary parabolic subspace, and this is a contradiction. We assume from now on that $v$ is nonzero.

Suppose now that $v$ is a vector in $V \backslash \Delta_{c}$. Choose a sequence $a_{1}, \ldots, a_{k}$ to satisfy conditions (i) and (ii) of Lemma 6.9, and furthermore choose the sequence to minimize $k$. Thus $\tilde{v}=a_{k} a_{k-1} \cdots a_{1}(v)$ has a nonpositive coefficient in its simpleroot expansion. Write $\tilde{c}$ for the Coxeter element $a_{k} a_{k-1} \cdots a_{1} c a_{1} \cdots a_{k-1} a_{k}$.

Write $\tilde{v}_{+}$for the vector $\sum_{i=0}^{n}\left[\tilde{v}: \alpha_{i}\right]_{+} \alpha_{i}$. By construction $\tilde{v}_{+}$is supported on a proper parabolic root subsystem. Therefore it has a unique $c^{\prime}$-cluster expansion involving only positive roots in the subsystem. (Here $c^{\prime}$ is the restriction of $\tilde{c}$ to the parabolic subgroup.) Property (4.3) and the fact that the only negative roots in $\Phi_{c}$ are negative simple roots then imply that $\tilde{v}$ has a unique $\tilde{c}$-cluster expansion and 
that this expansion writes $\tilde{v}$ as a nonnegative combination of positive roots from this parabolic and negative simple roots in its complement.

If $k=0$, then $\tilde{v}=v$ and we are done. If $k>0$, then $v$ has strictly positive simple-root coordinates. Because we chose $k$ to be minimal, $a_{k} \tilde{v}$ also has strictly positive simple-root coordinates. But $a_{k}$ equals some $s_{j}$, so $a_{k} \tilde{v}=s_{j} \tilde{v}$ differs from $\tilde{v}$ by a multiple of the simple root $\alpha_{j}$, and therefore $\tilde{v}$ has strictly positive simple-root coordinates except for the coordinate of $\alpha_{j}$. Thus the unique $\tilde{c}$-cluster expansion of $\tilde{v}$ is a nonnegative combination of positive roots whose $j$-th coordinate equals 0 and the root $-\alpha_{j}$. On all of these roots, the map $\sigma_{j}$ agrees with the map $s_{j}$. Property (4.7) thus implies that an $s_{j} \tilde{c} s_{j}$-cluster expansion of $s_{j} \tilde{v}$ is obtained by applying $s_{j}$ to each root in the $\tilde{c}$-cluster expansion of $\tilde{v}$. This $s_{j} \tilde{c} s_{j}$-cluster expansion of $s_{j} \tilde{v}$ is supported on positive roots, because otherwise property (4.3) implies a contradiction to the fact that $s_{j} \tilde{v}$ has strictly positive simple-root coordinates. We claim that this $s_{j} \tilde{c} s_{j}$-cluster expansion of $s_{j} \tilde{v}$ is unique. Any other $s_{j} \tilde{c} s_{j}$-cluster expansion of $s_{j} \tilde{v}$ is supported on positive roots for the same reason, so $\sigma_{j}$ agrees with $s_{j}$ on the other expansion as well. Thus property (4.7) implies that by applying $s_{j}$ to each root appearing in the other $s_{j} \tilde{c} s_{j}$-cluster expansion of $s_{j} \tilde{v}$, we obtain another $\tilde{c}$-cluster expansion of $\tilde{v}$, but we have already established that $\tilde{v}$ has a unique $\tilde{c}$-cluster expansion. This contradiction implies the uniqueness claim.

We now apply repeatedly the same argument to obtain a unique $c$-cluster expansion of $v$. The minimality of the sequence $a_{1}, \ldots, a_{k}$ guarantees that, at each step, we obtain an expansion supported only on positive roots so that the relevant simple reflection $s_{i}$ coincides with $\sigma_{i}$ on its support.

Finally, suppose $v \in \stackrel{\triangle}{ }_{c}$. Proposition 6.8 says that $v$ has a $c$-cluster expansion supported on roots in $\Lambda_{c}$, and that this is the unique $c$-cluster expansion of $v$ supported on such roots. It remains to show that $v$ has no $c$-cluster expansion whose support contains a negative simple root or a root outside of $U^{c}$. But if $v$ has a $c$-cluster expansion containing such a root, Proposition 3.12 implies that we can apply $\tau_{c}^{m}$, for some integer $m$, to one of the roots in the support of the $c$-cluster expansion to obtain a negative simple root. Thus there is a sequence $a_{1}, \ldots, a_{k}$ satisfying condition (i) of Lemma 6.9 such that the corresponding sequence of $\sigma_{i}$ takes one of the roots in the support of the $c$-cluster expansion to a negative simple. Choosing this sequence to minimize $k$, each of the $\sigma_{i}$ acts as the corresponding $s_{i}$ on each root, so $a_{k} \cdots a_{1}$ applied to the root yields a negative simple. Furthermore, the linear map $a_{k} \cdots a_{1}$ applied to all of the roots in the $c$-cluster expansion of $v$ yields an $a_{k} \cdots a_{1} c a_{1} \cdots a_{k}$-cluster expansion of $a_{k} \cdots a_{1} v$. By Property (4.3), one of the simpleroot coordinates of $a_{k} \cdots a_{1} v$ is strictly negative. This contradicts Proposition 6.6, because the latter implies that $a_{k} \cdots a_{1} v$ is in $\check{\Delta}_{c}$.

If $s$ is initial or final in $c$, then we extend the map $\sigma_{s}$ linearly on each cone of $\operatorname{Fan}_{c}(\Phi)$ to define a map (which we also call $\sigma_{s}$ ) on all of $V$. We extend $\tau_{c}$ in the same way. The following proposition is immediate by Proposition 3.3 and (4.7).

\section{Proposition 6.10.}

(1) If $s$ is initial or final in $c$ then $\sigma_{s}$ induces an isomorphism from $\operatorname{Fan}_{c}(\Phi)$ to $\operatorname{Fan}_{s c s}(\Phi)$, which restricts to an isomorphism $\operatorname{Fan}_{c}^{\mathrm{re}}(\Phi) \rightarrow \operatorname{Fan}_{\text {scs }}^{\mathrm{re}}(\Phi)$.

(2) The map $\tau_{c}$ induces an automorphism of $\operatorname{Fan}_{c}(\Phi)$, which restricts to an automorphism of $\operatorname{Fan}_{c}^{\mathrm{re}}(\Phi)$. 
Proposition 4.12 implies that the set $\Phi_{c}$ and the compatibility relation are preserved by rescaling $\Phi$, so the following proposition is immediate.

Proposition 6.11. If $\Phi$ and $\Phi^{\prime}$ are finite or affine root systems related by rescaling, then $\operatorname{Fan}_{c}(\Phi)$ coincides with $\operatorname{Fan}_{c}\left(\Phi^{\prime}\right)$.

Lemma 6.9 implies a description of $\Delta_{c}$ in terms of inequalities.

Proposition 6.12. The cone $\Delta_{c}$ is the set of points $v$ in $U^{c}$ satisfying the inequality $\left\langle s_{i_{1}} \cdots s_{i_{k}} \rho_{i_{k}}, v\right\rangle \geq 0$ for all sequences $s_{i_{1}}, \ldots, s_{i_{k}}$ of simple reflections with $k \leq M_{\Phi}$ such that $s_{i_{j}}$ is initial in the Coxeter element $s_{i_{j-1}} \cdots s_{i_{1}} c s_{i_{1}} \cdots s_{i_{j-1}}$ for all $i=1 \ldots k$.

Proof. Proposition 6.6 and the fact that $\Delta_{c}$ is contained in the cone of vectors with nonnegative simple-root coordinates for all $c$ imply that every $v \in \Delta_{c}$ satisfies the inequalities $\left\langle\rho_{i_{k}}, s_{i_{k}} \cdots s_{i_{1}} v\right\rangle \geq 0$. These are equivalent to $\left\langle s_{i_{1}} \cdots s_{i_{k}} \rho_{i_{k}}, v\right\rangle \geq 0$.

If $v$ is a nonzero vector in $V \backslash \AA_{c}$, then by Lemma 6.9 there is a sequence $s_{i_{1}}, \ldots, s_{i_{k}}$, with $k \leq M_{\Phi}$, satisfying the "initial or final" condition of the proposition such that the inequality $\left\langle\rho_{j}, s_{i_{k}} \cdots s_{i_{1}} v\right\rangle>0$ fails for some $j$. Choosing $k$ as small as possible, because $\alpha_{i_{k}}$ is the unique positive root that becomes negative under the action of $s_{i_{k}}$, we can take $j=i_{k}$. Thus the relative interior of $\Delta_{c}$ is separated from other points in $U^{c}$ by inequalities as described in the proposition. We conclude that $\Delta_{c}$ is defined by the given list of inequalities for $k \leq M_{\Phi}$.

The link of a ray $r$ in a simpicial fan is the subfan consisting of cones $C$ such that $r \nsubseteq C$ but the nonnegative linear span of $r \cup C$ is a cone of the fan. The star of $r$ is the subfan consisting of cones containing $r$. The following proposition is a restatement of Propositions 5.6, 5.13 and 6.12.

Proposition 6.13. Suppose $\Phi$ is of affine type and $c$ is a Coxeter element of $W$.

(1) The link of the ray spanned by $\delta$ is isomorphic, as a simplicial complex, to a join of boundary complexes of simplicial cyclohedra. There is a $(k-1)$ dimensional cyclohedron (and thus a $(k-2)$-dimensional boundary complex) for each rank-k component of $\Upsilon^{c}$.

(2) The union of the cones in the star of the ray spanned by $\delta$ is the cone $\Delta_{c}$. It is a closed polyhedral cone whose extreme rays are spanned by the simple roots of $\Upsilon^{c}$. Its defining inequalities are given by Proposition 6.12.

\section{EXCHANGEABLE ROOTS}

The definition of $\operatorname{Fan}_{c}(\Phi)$ depends only on when the compatibility degree is zero. In this section, we use more information about the compatibility degree (along with some extra information for cones contained in $\Delta_{c}$ ) to describe when roots in $c$-clusters can be exchanged, in the sense of the following definition.

Definition 7.1. Two distinct roots $\alpha$ and $\beta$ in $\Phi_{c}$ are said to be $c$-exchangeable if there exist $c$-clusters $C$ and $C^{\prime}$ with $\alpha \in C$, with $\beta \in C^{\prime}$, and with $C \backslash\{\alpha\}=C^{\prime} \backslash\{\beta\}$. Two distinct real roots in $\Phi_{c}$ are said to be c-real-exchangeable if there exist real clusters $C$ and $C^{\prime}$ with these properties.

If $\alpha, \beta \in \Phi_{c}$ are $c$-exchangeable, then they are not $c$-compatible. (If so, then $C \sqcup\{\beta\}$ is pairwise $c$-compatible for $C$ as in Definition 7.1. But $C$ is a $c$-cluster.)

Theorem 7.2. Suppose $\Phi$ is of affine type and $c$ is any Coxeter element. 
(1) Real roots $\alpha$ and $\beta$ in $\Phi_{c}^{\text {re }}$ are c-exchangeable if and only if

$$
(\alpha \| \beta)_{c}=1=(\beta \| \alpha)_{c} .
$$

(2) Real roots $\alpha$ and $\beta$ in $\Phi_{c}^{\mathrm{re}}$ are c-real-exchangeable if and only if they are $c$-exchangeable and $\alpha+\beta \notin \stackrel{\Delta}{c}_{c}$.

(3) Real roots $\alpha, \beta \in \Phi_{c}^{\mathrm{re}}$ that are c-exchangeable fail to be c-real-exchangeable if and only if $\alpha, \beta \in \Lambda_{c}^{\mathrm{re}}$ and $\operatorname{Supp}_{\Xi}(\alpha, \beta)$ is component-full.

(4) The imaginary root $\delta$ is not c-exchangeable with any other root.

Remark 7.3. In [17], exchangeability is defined by $(\alpha \| \beta)_{c}=1=(\beta \| \alpha)_{c}$ and then shown to be equivalent (in finite type) to the condition in Definition 7.1. We prefer the term "exchangeable" for the condition in Definition 7.1.

Despite Theorem 7.2(4), there are pairs of real roots that are in some sense $c$-exchangeable with $\delta$.

Definition 7.4. The pair $\{\alpha, \beta\}$ is c-exchangeable with $\delta$ if there exists a (necessarily imaginary) $c$-cluster $C$ with $\delta \in C$ and a (necessarily real) $c$-cluster $C^{\prime}$ with $\alpha, \beta \in C^{\prime}$, such that $C \backslash\{\delta\}=C^{\prime} \backslash\{\alpha, \beta\}$.

Theorem 7.5. Suppose $\Phi$ is of affine type, but not of type $A_{2 k}^{(2)}$ and suppose $c$ is any Coxeter element. For each $\alpha \in \Phi_{c}$, the following are equivalent.

(1) $(\alpha \| \delta)_{c}=1=(\delta \| \alpha)_{c}$.

(2) There exists $\beta \in \Phi_{c}$ such that the pair $\{\alpha, \beta\}$ is c-exchangeable with $\delta$.

When these equivalent conditions hold, $\alpha$ is in the $\tau_{c}$-orbit of a negative simple root $-\alpha_{j}$ such that there exists a diagram automorphism taking $\alpha_{j}$ to $\alpha_{\mathrm{aff}}$.

Remark 7.6. Theorem 7.5 stops short of describing which pairs $\{\alpha, \beta\}$ are $c$ exchangeable with $\delta$. The simplest characterization one might propose is that $\{\alpha, \beta\}$ are $c$-exchangeable with $\delta$ if and only if $\alpha$ and $\beta$ are $c$-compatible and both satisfy the conditions of Theorem 7.5. However, this is already false in type $A_{2}^{(1)}$, as we now explain. Recall that $A_{2}^{(1)}$ has rank 3, take $c$ as in Table 2.1, and recall that each $\alpha_{i}$ equals $\alpha_{i}^{\vee}$ and that $\delta=\delta^{\vee}=\alpha_{1}+\alpha_{2}+\alpha_{3}$. Setting $\alpha=-\alpha_{1}$ and $\beta=-\alpha_{2}$, indeed $\alpha$ and $\beta$ are $c$-compatible, $(\alpha \| \delta)_{c}=1=(\delta \| \alpha)_{c}$, and $(\beta \| \delta)_{c}=1=(\delta \| \beta)_{c}$. Diagram automorphisms act transitively on the simple roots. However, there are only two real $c$-clusters that contain $\alpha$ and $\beta$, namely $\left\{\alpha, \beta,-\alpha_{3}\right\}$ and $\left\{\alpha, \beta, \alpha_{3}\right\}$, and we calculate $\left(-\alpha_{3} \| \delta\right)_{c}=1$ and $\left(\alpha_{3} \| \delta\right)_{c}=\left(\tau_{c} \alpha_{3} \| \tau_{c} \delta\right)_{c}=\left(-\alpha_{3} \| \delta\right)_{c}=1$.

Example 7.7. We give two simple examples to illustrate why Theorem 7.5 must exclude type $A_{2 k}^{(2)}$. First, type $A_{2}^{(2)}$ has rank 2 , so any real $c$-cluster is a $c$-exchangeable pair with $\delta=\alpha_{1}+2 \alpha_{2}$ (taking aff $=2$ ). Thus $\left\{-\alpha_{1},-\alpha_{2}\right\}$ is an exchangeable pair, but $\left(-\alpha_{2} \| \delta\right)_{c}=2$ for either choice of $c$. Second, consider $A_{4}^{(2)}$ with aff $=3$, so that $\delta=\alpha_{1}+2 \alpha_{2}+2 \alpha_{3}$, and take $c=s_{1} s_{2} s_{3}$. Then $\operatorname{Fan}_{c}(\Phi)$ is the same as the fan shown in Fig. 6.1. The smallest triangle shown has vertices $-\alpha_{1}$ (red), $-\alpha_{2}$ (green), and $-\alpha_{3}$ (blue), and is adjacent to a triangle with $-\alpha_{1}, \alpha_{2}$ (cyan), and $-\alpha_{3}$. Since $\alpha_{2}$ is compatible with $\delta$, the pair $\left\{-\alpha_{1},-\alpha_{3}\right\}$ is $c$-exchangeable with $\delta$, but $\left(-\alpha_{3} \| \delta\right)_{c}=2$.

We now prepare to prove Theorems 7.2 and 7.5. The following proposition is immediate by Proposition 5.4.

Proposition 7.8. Suppose $\Phi$ is of affine type and let $\alpha, \beta \in \Phi_{c}$. 
(1) If $s$ is an initial or final reflection in $c$ then $\alpha$ and $\beta$ are c-exchangeable if and only if $\sigma(\alpha)$ and $\sigma(\beta)$ are scs-exchangeable.

(2) $\alpha$ and $\beta$ are $c$-exchangeable if and only if $\tau_{c}(\alpha)$ and $\tau_{c}(\beta)$ are $c$-exchangeable. The same assertions hold for real c-exchangeability. Similarly, a pair $\{\alpha, \beta\}$ is cexchangeable with $\delta$ if and only if $\left\{\tau_{c} \alpha, \tau_{c} \beta\right\}$ is c-exchangeable with $\delta$ and if and only if $\left\{\sigma_{s} \alpha, \sigma_{s} \beta\right\}$ is scs-exchangeable with $\delta$.

Parts of the proofs in this section are deferred to Section 8, where they are checked using the classifications of affine root systems. One can reduce the number of types that must be checked by performing a rescaling. Proposition 6.11 says that, if $\Phi^{\prime}$ is a rescaling of $\Phi$, then $\operatorname{Fan}_{c}(\Phi)=\operatorname{Fan}_{c}\left(\Phi^{\prime}\right)$. In particular, the notions of $c$-exchangeability with respect to $\Phi$ and $\Phi^{\prime}$ coincide. The condition $(\alpha \| \beta)_{c}=1=$ $(\beta \| \alpha)_{c}$ is also, in most cases, robust under rescaling.

Proposition 7.9. Suppose $\Phi$ and $\Phi^{\prime}$ are affine root systems related by a rescaling $\beta \mapsto \beta^{\prime}=\lambda_{\beta} \beta$. Then for real roots $\alpha$ and $\beta$ in $\Phi_{c}^{\text {re }}$ :

(1) the conditions $(\alpha \| \beta)_{c}=1=(\beta \| \alpha)_{c}$ and $\left(\alpha^{\prime} \| \beta^{\prime}\right)_{c}=1=\left(\beta^{\prime} \| \alpha^{\prime}\right)_{c}$ are equivalent;

(2) the conditions $(\alpha \| \delta)_{c}=1=(\delta \| \alpha)_{c}$ and $\left(\alpha^{\prime} \| \delta^{\prime}\right)_{c}=1=\left(\delta^{\prime} \| \alpha^{\prime}\right)_{c}$ are equivalent except possibly when $\Phi$ or $\Phi^{\prime}$ is of type $A_{2 k}^{(2)}$.

Proof. If $(\alpha \| \beta)_{c}=1=(\beta \| \alpha)_{c}$, then $\frac{\lambda_{\alpha}}{\lambda_{\beta}}\left(\alpha^{\prime} \| \beta^{\prime}\right)_{c}=1=\frac{\lambda_{\beta}}{\lambda_{\alpha}}\left(\beta^{\prime} \| \alpha^{\prime}\right)_{c}$ by Proposition 4.12. Thus $\frac{\lambda_{\beta}}{\lambda_{\alpha}}=\left(\alpha^{\prime} \| \beta^{\prime}\right)_{c}$ and $\frac{\lambda_{\alpha}}{\lambda_{\beta}}=\left(\beta^{\prime} \| \alpha^{\prime}\right)_{c}$. In particular, both $\frac{\lambda_{\beta}}{\lambda_{\alpha}}$ and $\frac{\lambda_{\alpha}}{\lambda_{\beta}}$ are positive integers. Therefore $\lambda_{\alpha}=\lambda_{\beta}$ and $\left(\alpha^{\prime} \| \beta^{\prime}\right)_{c}=1=\left(\beta^{\prime} \| \alpha^{\prime}\right)_{c}$. The symmetric argument proves the converse.

For the second assertion, by (4.6), we can assume that $\alpha \notin \Lambda_{c}$. Proposition 3.12 says that $\alpha$ is in the $\tau_{c}$-orbit of a negative simple root. Thus by (4.8), we may as well take $\alpha$ to be $-\alpha_{i}$ for some $i$. In this case $(\alpha \| \delta)_{c}=\left[\delta: \alpha_{i}\right]$ by (4.3) and $(\delta \| \alpha)_{c}=\left[\delta^{\vee}: \alpha_{i}^{\vee}\right]$ by (4.4). If neither $\Phi$ nor $\Phi^{\prime}$ is of type $A_{2 k}^{(2)}$, then inspection of the classification of root system of affine type (see [24, Tables Aff 1-3]), reveals that the property that $\left[\delta: \alpha_{i}\right]=1=\left[\delta^{\vee}: \alpha_{i}^{\vee}\right]$ is preserved by rescaling.

We now begin the proof of Theorem 7.2(1). We prove all of one direction of the assertion and reduce the other direction to a proposition that we will prove type-by-type in Section 8.

Proof of Theorem 7.2(1). As in the proof Theorem 6.2, one could prove this for finite and affine type together by induction on rank, but instead, we use the finite case, which follows from [17, Proposition 3.5] and [26, Proposition 4.10].

Suppose $\alpha, \beta$ in $\Phi_{c}^{\text {re }}$ are $c$-exchangeable. If they are $c$-real-exchangeable, then let $C$ and $C^{\prime}$ be real $c$-clusters with $\alpha \in C$, with $\beta \in C^{\prime}$, and with $C \backslash\{\alpha\}=$ $C^{\prime} \backslash\{\beta\}$. Proposition 5.14(3) says that $C$ contains at least two roots in the $\tau_{c^{-}}$ orbits of negative simple roots. Thus $C \backslash\{\alpha\}$ contains at least one root in the $\tau_{c}$-orbit of a negative simple root. By Proposition 7.8(2) and (4.8), we may as well assume that $C \backslash\{\alpha\}$ contains a negative simple root $-\alpha_{i}$. Then Proposition 5.4 implies that $C \backslash\left\{-\alpha_{i}\right\}$ and $C^{\prime} \backslash\left\{-\alpha_{i}\right\}$ are $c^{\prime}$-clusters in a proper parabolic root subsystem $\Phi^{\prime}$ of $\Phi$, where $c^{\prime}$ is the restriction of $c$. The finite case result then says that $(\alpha \| \beta)_{c^{\prime}}=1=(\beta \| \alpha)_{c^{\prime}}$. Proposition 4.15 implies that $(\alpha \| \beta)_{c}=1=(\beta \| \alpha)_{c}$.

If $\alpha$ and $\beta$ are not $c$-real-exchangeable, then there exist $c$-clusters $C$ and $C^{\prime}$, not both real, with $\alpha \in C$, with $\beta \in C^{\prime}$, and with $C \backslash\{\alpha\}=C^{\prime} \backslash\{\beta\}$. But then $C$ and $C^{\prime}$ have the same cardinality, so Proposition 5.14 implies that both are imaginary and 


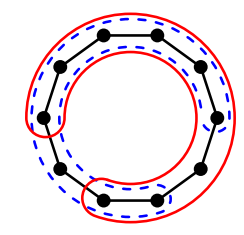

Figure 7.1. One of the cases discussed in the proof of Theorem 7.2.

contained in $\Lambda_{c}$. In particular $(\alpha \| \beta)_{c}=(\alpha \circlearrowright \beta)_{c}$ and $(\beta \| \alpha)_{c}=(\beta \circlearrowright \alpha)_{c}$. Since $\alpha$ and $\beta$ are $c$-exchangeable, they are not $c$-compatible, so by symmetry, to show that $(\alpha \| \beta)_{c}=1=(\beta \| \alpha)_{c}$, it suffices to rule out $(\alpha \circlearrowright \beta)_{c}=2$.

Suppose $(\alpha \circlearrowright \beta)_{c}=2$. Then $\alpha$ and $\beta$ are overlapping or adjacent on both sides and $\alpha$ has two distinct adjacent roots. Let $\ell$ be the number of simple roots of $\Upsilon^{c}$ in the overlap of $\operatorname{Supp}_{\Xi}(\alpha)$ and $\operatorname{Supp}_{\Xi}(\beta)$ on one side and let $m$ be the number of simple roots in the overlap on the other side. Let $p$ be the number of simple roots (in the component) not contained in or adjacent to $\operatorname{Supp}_{\Xi}(\alpha)$ and let $q$ be the number of simple roots in that are not contained in or adjacent to $\operatorname{Supp}_{\Xi}(\beta)$. Using Proposition 5.12, we see that the maximum size of a set of pairwise $c$-compatible real roots in the component, $c$-compatible with both $\alpha$ and $\beta$, is $\ell+m+p+q$, which is 4 less than the size of the component if $\beta$ has two adjacent roots, or 3 less if $\beta$ has one adjacent root. The maximum size of a set of pairwise $c$-compatible roots in each other component is 1 less than the size of the component. Since by Proposition 2.12, the rank of $\Upsilon^{c}$, minus the number of components, is $n-2$, the maximum size of a set of pairwise $c$-compatible real roots in $\Upsilon^{c}, c$-compatible with both $\alpha$ and $\beta$, is $n-4$. Allowing $\delta$ in the set, we conclude that $C \backslash\{\alpha\}$ can contain at most $n-3$ roots. This contradicts Proposition 5.14(5), so $(\alpha \circlearrowright \beta)_{c} \neq 2$.

We have proved one direction of Theorem 7.2(1). To prove the remaining direction of Theorem 7.2(1), suppose $(\alpha \| \beta)_{c}=1=(\beta \| \alpha)_{c}$. If there exists a root $\gamma$ in the $\tau_{c}$-orbit of some negative simple root $-\alpha_{i}$ that is $c$-compatible with both $\alpha$ and $\beta$, then by Proposition 7.8(2) and (4.8), we may as well assume that some $-\alpha_{i}$ is $c$-compatible with both $\alpha$ and $\beta$. By (4.3), $\alpha$ and $\beta$ are in a proper standard parabolic root subsystem of $\Phi$ and Proposition 4.15 says that their compatibility degree in the root subsystem is 1 in both directions. By the result for finite type, $\alpha$ and $\beta$ are exchangeable in the root subsystem. Adjoining $-\alpha_{i}$ to the two relevant clusters, we see that $\alpha$ and $\beta$ are $c$-exchangeable in $\Phi_{c}$ as well.

Suppose that $\alpha$ and $\beta$ are both in $\Lambda_{c}^{\mathrm{re}}$. Then there are two possibilities allowed by the equality $(\alpha \| \beta)_{c}=1=(\beta \| \alpha)_{c}$. One possibility is that $\alpha$ and $\beta$ are overlapping or adjacent on only one side. But then $\operatorname{Supp}_{\Xi}(\alpha, \beta)$ is not component-full, so Proposition 5.9 implies that there is a root in the $\tau_{c}$-orbit of a negative simple root that is $c$-compatible with both $\alpha$ and $\beta$, so we have already covered this possibility. The other possibility, pictured in Fig. 7.1 with the same drawing conventions as in Table 4.1, is that $\operatorname{Supp}_{\Xi}(\alpha)$ and $\operatorname{Supp}_{\Xi}(\beta)$ each omit a single simple root of the component of $\Upsilon^{c}$ that contains them both. In this case, let $p$ and $q$ be the numbers of simple roots in the two components of $\operatorname{Supp}_{\Xi}(\alpha) \cap \operatorname{Supp}_{\Xi}(\beta)$. Using Proposition 5.12, we can construct a set of $p+q$ pairwise $c$-compatible real roots in the component, each $c$-compatible with both $\alpha$ and $\beta$. This is 2 less than the size of the component. In each other component of size $k$, we can find a set $k-1$ of 
pairwise $c$-compatible roots. Since the rank of $\Upsilon^{c}$ minus the number of components is $n-2$, we have constructed a set $C$ of $n-3$ pairwise $c$-compatible real roots in $\Upsilon^{c}$, each $c$-compatible with both $\alpha$ and $\beta$. By Proposition 5.14 and (4.6), the sets $C \cup\{\alpha, \delta\}$ and $C \cup\{\beta, \delta\}$ witness that $\alpha$ and $\beta$ are $c$-exchangeable.

The remaining case is where one or both of $\alpha$ and $\beta$ are not in $\Lambda_{c}^{\mathrm{re}}$, or equivalently by Proposition 3.12, one or both is in the $\tau_{c}$-orbit of a negative simple root. By Proposition 2.9, Proposition 7.8(1), Lemma 3.11, and (4.7), we can choose one Coxeter element $c$ in each conjugacy class for which to check this remaining case. Without loss of generality (by switching the names $\alpha$ and $\beta$ for the roots), $\alpha$ is in the $\tau_{c}$-orbit of a negative simple root, and by Proposition 7.8(2) and (4.8), we may as well assume that $\alpha$ is a negative simple root $-\alpha_{j}$. If $K\left(\gamma_{c}, \beta\right)>0$, then since $U^{c}$ is defined by the equation $K\left(\gamma_{c}, \cdot\right)=0$ and since $\left[\beta: \alpha_{j}\right]=1$, Proposition 3.12(5) says that $\beta$ is in an infinite $\tau_{c}$-orbit, so Proposition 3.12(8) says that $\tau_{c}^{m}(\beta)$ is a negative simple for some $m<0$. Proposition 3.12(8) also says that $K\left(\gamma_{c}, \tau_{c}^{m}\left(-\alpha_{j}\right)\right)<0$. Thus, up to applying $\tau_{c}$ several times and then switching $\alpha$ and $\beta$ and changing $j$, we can assume that $\alpha=-\alpha_{j}$ and $K\left(\gamma_{c}, \beta\right) \leq 0$. Since we have already handled the case where some root in the $\tau_{c}$-orbit of some negative simple root is $c$-compatible with both $\alpha$ and $\beta$, by (4.3) and (4.8) we may as well assume that $\operatorname{Supp}_{\Pi}\left(\tau_{c}^{m}(\alpha), \tau_{c}^{m}(\beta)\right)$ is full for every integer $m$. In particular, $\beta$ has full support. By Proposition 4.11, we can rewrite the hypothesis $(\alpha \| \beta)_{c}=1=(\beta \| \alpha)_{c}$ as $(\alpha \| \beta)_{c}=1=\left(\alpha^{\vee} \| \beta^{\vee}\right)_{c}$, and since $\alpha=-\alpha_{j}$, this is $\left[\beta: \alpha_{j}\right]=\left[\beta^{\vee}: \alpha_{j}^{\vee}\right]=1$. We complete the proof of Theorem 7.2(1) by verifying that this final case cannot occur.

Proposition 7.9 allows us to restrict our attention to the standard affine root systems. Thus the following proposition completes the proof.

Proposition 7.10. Suppose $\Phi$ is of standard affine type. In each conjugacy class of Coxeter element, there exists $c$ such that the following assertion holds: For each $j \in\{1, \ldots, n\}$, there does not exist $\beta \in \Phi_{c}^{\mathrm{re}}$ with full support such that

(1) $\left[\beta: \alpha_{j}\right]=\left[\beta^{\vee}: \alpha_{j}^{\vee}\right]=1$,

(2) $\operatorname{Supp}_{\Pi}\left(\tau_{c}^{m}\left(-\alpha_{j}\right), \tau_{c}^{m}(\beta)\right)$ is full for all integers $m$.

(3) $K\left(\gamma_{c}, \beta\right) \leq 0$.

Proposition 7.10 is proved in Section 8. We proceed to prove the remainder of Theorem 7.2. We will use Theorem 7.2(1) to prove the remaining parts, but when we prove Proposition 7.10 (and thus complete the proof of Theorem 7.2(1)), we do not use Theorem 7.2.

Proof of Theorem 7.2(2-3). Suppose $\alpha$ and $\beta$ are $c$-exchangeable roots but are not $c$-real-exchangeable. In the proof of Theorem 7.2(1), we showed that in this case $\alpha, \beta \in \Lambda_{c}^{\text {re }}$. We want to show that $\operatorname{Supp}_{\Xi}(\alpha, \beta)$ is component-full and that $\alpha+\beta$ is in $\Delta_{c}$. If $\operatorname{Supp}_{\Xi}(\alpha, \beta)$ is not component-full, then $R=\{\alpha, \beta\}$ satisfies the hypotheses of Proposition 5.9, so there exists a root $\tau_{c}^{k}\left(-\alpha_{j}\right)$ for some $j \in\{1, \ldots, n\}$ and $k \in \mathbb{Z}$ that is $c$-compatible with both $\alpha$ and $\beta$. Thus by (4.8), $\tau_{c}^{-k} \alpha$ and $\tau_{c}^{-k} \beta$ are both $c$-compatible with $-\alpha_{j}$, and thus by (4.3), both are in the proper parabolic root subsystem deleting $\alpha_{j}$. Writing $c^{\prime}$ for the restriction of $c$ to the parabolic subgroup, Theorem 7.2(1) and Proposition 4.15 imply that $\left(\tau_{c}^{-k} \alpha \| \tau_{c}^{-k} \beta\right)_{c^{\prime}}=1=\left(\tau_{c}^{-k} \beta \| \tau_{c}^{-k} \alpha\right)_{c^{\prime}}$. The finite-type result implies that $\tau_{c}^{-k} \alpha$ and $\tau_{c}^{-k} \beta$ are $c^{\prime}$-exchangeable in the parabolic subgroup. Inserting $-\alpha_{j}$ into the $c^{\prime}$ clusters that witness the $c^{\prime}$-exchangeability, we see that $\tau_{c}^{-k} \alpha$ and $\tau_{c}^{-k} \beta$ are $c^{\prime}$-realexchangeable. Now Proposition 7.8(2) implies that $\alpha$ and $\beta$ are $c$-real-exchangeable. 
This contradiction shows that $\operatorname{Supp}_{\Xi}(\alpha, \beta)$ is component-full. Therefore $\alpha+\beta-\delta$ is a nonnegative linear combination of positive roots of $\Upsilon^{c}$. Since $\delta$ is in $\stackrel{\Delta}{c}_{c}$ and the positive roots of $\Upsilon^{c}$ are in $\Delta_{c}$, we conclude that $\alpha+\beta$ is in $\AA_{c}$.

We now continue to assume that $\alpha$ and $\beta$ are $c$-exchangeable but now assume that they are $c$-real-exchangeable. To complete the proof, we need to show that $\alpha+\beta \notin \dot{\Delta}_{c}$ and to show that if $\alpha, \beta \in \Lambda_{c}^{\text {re }}$, then $\operatorname{Supp}_{\Xi}(\alpha, \beta)$ is not component-full. Let $C$ and $C^{\prime}$ be real $c$-clusters with $\alpha \in C$, with $\beta \in C^{\prime}$, and with $C \backslash\{\alpha\}=$ $C^{\prime} \backslash\{\beta\}$. Proposition 5.14(3) says that $C$ contains at least two roots in the $\tau_{c^{-}}$ orbits of negative simple roots. Thus $C \backslash\{\alpha\}$ contains at least one root in the $\tau_{c^{-}}$-orbit of a negative simple root. Therefore, Properties (4.3) and (4.8) imply that $\operatorname{Supp}_{\Pi}\left(\tau_{c}^{k}(\alpha), \tau_{c}^{k}(\beta)\right)$ is not full for some integer $k$.

Suppose for the sake of contradiction that $\alpha, \beta \in \Lambda_{c}^{\mathrm{re}}$ and that $\operatorname{Supp}_{\Xi}(\alpha, \beta)$ is component-full. Then by Proposition $2.15, \operatorname{Supp}_{\Xi}\left(\tau_{c}^{k}(\alpha), \tau_{c}^{k}(\beta)\right)$ is also componentfull. But then $\tau_{c}^{k}(\alpha)+\tau_{c}^{k}(\beta)$ is $\delta$ plus a nonnegative combination of positive roots, and since $\operatorname{Supp}_{\Pi}(\delta)$ is full, we conclude that $\operatorname{Supp}_{\Pi}\left(\tau_{c}^{k}(\alpha), \tau_{c}^{k}(\beta)\right)$ is full, and this is our contradiction.

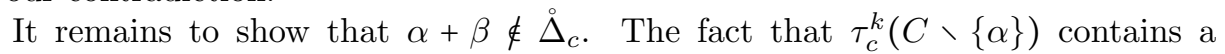
negative simple root for some $k$ means that, reading indices modulo $n$, the set $\sigma_{1} \cdots \sigma_{n k}(C \backslash\{\alpha\})$, if $k>0$, or $\sigma_{-n k+1} \cdots \sigma_{0}(C \backslash\{\alpha\})$, if $k<0$, contains a negative simple root. Choose $\ell$ with the smallest absolute value such that $\sigma_{1} \cdots \sigma_{\ell}(C \cup\{\beta\})$, if $k \geq 0$, or $\sigma_{-\ell} \cdots \sigma_{0}(C \cup\{\beta\})$, if $k<0$, contains a negative simple root, and write $\Sigma$ for $\sigma_{1} \cdots \sigma_{\ell}$ or $\sigma_{-\ell} \cdots \sigma_{0}$. Since we choose $\ell$ with smallest absolute value, since $\sigma_{i}$ acts as $s_{i}$ on positive roots, and since $\pm \alpha_{i}$ are the only roots whose sign changes under the action of $s_{i}$, we see that $(\Sigma(C \cup\{\beta\})) \cap(-\Pi)=\left\{-\alpha_{\ell}\right\}$. If neither $\Sigma(\alpha)$ nor $\Sigma(\beta)$ is $-\alpha_{\ell}$, then $\Sigma(\alpha)$ and $\Sigma(\beta)$ are both in the standard parabolic subgroup of $\Phi$ that omits the simple root $\alpha_{\ell}$. If $\Sigma(\alpha)$ or $\Sigma(\beta)$ is $-\alpha_{\ell}$, then, since we are assuming that $\alpha$ and $\beta$ are $c$-exchangeable and have proved that $(\alpha \| \beta)_{c}=1=(\beta \| \alpha)_{c}$, the other of $\Sigma(\alpha)$ or $\Sigma(\beta)$ has $\alpha_{\ell}$-coordinate 1 in its simple-root expansion by (4.3). In either case, $\Sigma(\alpha)+\Sigma(\beta)$ is in the span of $\Pi \backslash\left\{\alpha_{\ell}\right\}$. Write $\tilde{c}$ for $s_{1} \cdots s_{\ell} c s_{\ell} \cdots s_{1}$, if $\ell \geq 0$, or for $s_{-\ell} \cdots s_{0} c s_{0} \cdots s_{\ell}$, if $\ell<0$. Since all vectors in $\triangle_{\tilde{c}}$ have strictly positive simple-root coordinates, we see that $\Sigma(\alpha)+\Sigma(\beta)$ is not in $\stackrel{\Delta}{\tilde{c}}_{\tilde{c}}$. Since each $\sigma_{i}$ that makes up $\Sigma$ acts as $s_{i}$, Proposition 6.6 implies that $\alpha+\beta$ is not in $\AA_{c}$. This completes the proof of Theorem 7.2(2-3).

Proof of Theorem 7.2(4). Suppose $C$ is a $c$-cluster with $\delta \in C$. Then by definition $C$ is an imaginary cluster, so Proposition 5.14 says that $|C|=n-1$. If $\beta$ is any real root in $\Phi_{c}^{\text {re }}$, then $(C \backslash\{\delta\}) \cup\{\beta\}$ has $\leq n-1$ roots, and thus is not a real cluster by Proposition 5.14. It is also not an imaginary cluster, since it does not contain $\delta$. We see that $\beta$ and $\delta$ are not exchageable.

Finally, we reduce Theorem 7.5 to a proposition that will be proved in Section 8 .

Proof of Theorem 7.5. Suppose $(\alpha \| \delta)_{c}=1=(\delta \| \alpha)_{c}$. Since in particular $\alpha$ and $\delta$ are not $c$-compatible, by Proposition 5.6, $\alpha$ is in the $\tau_{c}$-orbit of a negative simple root. As in the proof of Theorem 7.2(1), we can assume that $\alpha=-\alpha_{j}$ for some $j$. As before, the assumption that $(\alpha \| \delta)_{c}=1=(\delta \| \alpha)_{c}$ means that $\left[\delta: \alpha_{j}\right]=1=\left[\delta^{\vee}: \alpha_{j}^{\vee}\right]$. Inspection of the classification (for example in [24, Tables Aff 1-3]) shows that $\left[\delta: \alpha_{j}\right]=1=\left[\delta^{\vee}: \alpha_{j}^{\vee}\right]$ happens if and only if $\alpha_{j}=\alpha_{\text {aff }}$ or there is some diagram 
automorphism taking $\alpha_{j}$ to $\alpha_{\text {aff }}$. Since we will be proving this direction of the theorem for all possible $c$, we may as well take $\alpha_{j}=\alpha_{\text {aff }}$.

As before, let $\beta_{1}, \ldots, \beta_{n-2}$ be the simple roots of $\Upsilon_{\text {fin }}^{c}$. Then the set $\Omega^{c}$ is a collection of pairwise $c$-compatible roots in $\Lambda_{c}^{\text {re }}$. (Within each component they are all nested.) In particular, each of these is $c$-compatible with $\alpha=-\alpha_{\text {aff }}$. By Theorem 5.5 and Proposition 5.14(1), $\Omega^{c} \cup\{\alpha\}$ is contained in some real cluster $C^{\prime}=\Omega^{c} \cup\{\alpha, \beta\}$ for some $\beta$. Writing $C$ for the cluster $\Omega^{c} \cup\{\delta\}$, we have $C \backslash\{\delta\}=$ $C^{\prime} \backslash\{\alpha, \beta\}$. That is, $\{\alpha, \beta\}$ is $c$-exchangeable with $\delta$.

Conversely, given $\alpha \in \Phi_{c}$, suppose there exists $\beta \in \Phi_{c}$ such that the pair $\{\alpha, \beta\}$ is $c$-exchangeable with $\delta$. Specifically, suppose $C$ is an imaginary $c$-cluster and $C^{\prime}$ is a $c$-cluster with $\alpha, \beta \in C^{\prime}$, such that $C \backslash\{\delta\}=C^{\prime} \backslash\{\alpha, \beta\}$. We want to prove that $(\alpha \| \delta)_{c}=1=(\delta \| \alpha)_{c}$. By Propositions 4.12 and 7.9, it is enough to check the theorem only for the standard affine root systems. By Proposition 7.8 and (4.7), it is enough to check only one choice of $c$ in each conjugacy class.

Because $C \backslash\{\delta\}=C^{\prime} \backslash\{\alpha, \beta\}$, in particular, $\alpha \notin C$. Since $C$ is a cluster (a maximal set of pairwise $c$-compatible roots in $\Phi_{c}$ ), and $\alpha$ is compatible with any root in $C \backslash\{\delta\}$, we deduce that the root $\alpha$ is not $c$-compatible with $\delta$. In particular Proposition 5.6 says that $\alpha$ is in the $\tau_{c}$-orbit of a negative simple root $-\alpha_{j}$. By (4.8) and the fact that $\tau_{c}$ fixes $\delta$, we may as well assume that $\alpha=-\alpha_{j}$. By (4.3) and (4.4), the assertion that $\left(-\alpha_{j} \| \delta\right)_{c}=1=\left(\delta \|-\alpha_{j}\right)_{c}$ is equivalent to the assertion that $\left[\delta: \alpha_{j}\right]=1=\left[\delta^{\vee}: \alpha_{j}^{\vee}\right]$. As mentioned above, the latter assertion is equivalent to the assertion that $\alpha_{j}=\alpha_{\text {aff }}$ or there is some diagram automorphism taking $\alpha_{j}$ to $\alpha_{\text {aff }}$. The roots $C \backslash\{\delta\}$ are all in $\Lambda_{c}^{\text {re }}$ by Proposition 5.6. Also, Proposition 5.14 implies that $C \backslash\{\delta\}$ contains exactly $n-2$ roots, and these roots are linearly independent. In particular, $\left|\operatorname{Supp}_{\Xi}(C \backslash\{\delta\})\right| \geq n-2$. As mentioned above in the proof of most of Theorem 7.2(1), the rank of $\Upsilon^{c}$, minus the number of components, is $n-2$, so Lemma 5.10 says that $\operatorname{Supp}_{\Xi}(C \backslash\{\delta\})$ consists of all but one simple root in each component of $\Upsilon^{c}$. Each root in $C \backslash\{\delta\}$ is $c$-compatible with $-\alpha_{j}$, so $\alpha_{j} \notin \operatorname{Supp}_{\Pi}(C \backslash\{\delta\})$, which further implies that $\alpha_{j} \notin \operatorname{Supp}_{\Pi}\left(\operatorname{Supp}_{\Xi}(C \backslash\{\delta\})\right)$. In Section 8 , we complete the proof by proving the following proposition.

Proposition 7.11. Suppose $\Phi$ is a standard affine root system. Then there exists a Coxeter element $c$ such that the following assertion holds: If $R$ is a set of simple roots of $\Upsilon^{c}$ consisting of all but one simple root in each component of $\Upsilon^{c}$, and if $-\alpha_{j}$ is a simple root of $\Phi$ with $\alpha_{j} \notin \operatorname{Supp}_{\Pi}(R)$, then $\alpha_{j}=\alpha_{\mathrm{aff}}$ or there is some diagram automorphism taking $\alpha_{j}$ to $\alpha_{\mathrm{aff}}$.

\section{TYPE-BY-TYPE ARGUMENTS FOR EXCHANGEABILITY}

In this section, we prove Propositions 7.10 and 7.11 by a type-by-type check in the classification of affine root systems. Neither proof relies on results of Section 7 .

Proof of Proposition 7.10. We argue type-by-type, and we take $c=s_{1} \cdots s_{n}$ according to the labeling in Table 2.1. Exceptional types are checked by computer.

Recall that $\alpha_{\text {aff }}=\alpha_{n}$ is every case. Since $\Phi$ is of standard affine type, every root $\beta$ of $\Phi$ is of the form $\beta^{\prime}+k \delta$ for some $\beta^{\prime} \in \Phi_{\text {fin }}$. In every case, we assume conditions (1) and (3). To eliminate cases, we use the observation that no root in $\Phi_{c}^{\mathrm{re}}$ is in $U^{c}$ and has $\operatorname{Supp}_{\Xi}(\beta)$ component-full. Thus for each possible $\beta$, we either prove that $\beta \in U^{c}$ and $\operatorname{Supp}_{\Xi}(\beta)$ is component-full or we prove that (2) fails. In the simply laced cases, we scale $\Phi$ so that roots and co-roots coincide. We rewrite condition (3) 
as $\left\langle\phi_{c}, \beta\right\rangle \leq 0$ for $\phi_{c}$ as in Lemma 2.11. We have $\beta \in U^{c}$ if and only if $\left\langle\phi_{c}, \beta\right\rangle=0$. Throughout, we use the fact that $\delta$ is fixed by $c$. We also use the fact that if each of the roots $\beta, c(\beta), \ldots, c^{p}(\beta)$ is positive for some $p$, then $c^{p}(\beta)=\tau_{c}^{p}(\beta)$.

Case $A_{n-1}^{(1)}$ : In this case, Lemma 2.11 implies that, up to positive scaling, $\phi_{c}$ is $\rho_{n}-\rho_{1}$. Thus we are looking for $\beta$ with $\left[\beta: \alpha_{1}\right] \geq\left[\beta: \alpha_{n}\right]$. A root $\beta$ with full support, at least one simple-root coordinate $=1$ and $\left[\beta: \alpha_{1}\right] \geq\left[\beta: \alpha_{n}\right]$ is of the form $\beta^{\prime}+\delta$ for $\beta^{\prime} \in \Phi_{\text {fin }}^{+}$or $-\beta^{\prime}+2 \delta$ for $\beta^{\prime} \in \Phi_{\{2, \ldots, k\}}^{+}$or $\beta^{\prime} \in \Phi_{\{k+1, \ldots, n-1\}}^{+}$. But in the latter case, $\beta \in U^{c}$ and $\operatorname{Supp}_{\Xi}(\beta)$ is component-full. Similarly, if $\beta=\beta^{\prime}+\delta$ for $\beta^{\prime} \in \Phi_{\text {fin }}^{+}$such that $\left[\beta^{\prime}: \alpha_{1}\right]=0$, again $\beta \in U^{c}$ and $\operatorname{Supp}_{\Xi}(\beta)$ is component-full. Thus we can assume that $\beta=\beta^{\prime}+\delta$ for $\beta^{\prime} \in \Phi_{\text {fin }}^{+}$and $\left[\beta^{\prime}: \alpha_{1}\right]=1$.

We write $\beta^{\prime}=\alpha_{1}+\cdots+\alpha_{\ell}+\alpha_{k+1}+\cdots+\alpha_{k+m}$, and without loss of generality, we can take $m<\ell$. (Otherwise, rename the roots $\alpha_{2}, \ldots, \alpha_{k}$ as $\alpha_{k+1}, \ldots, \alpha_{n-1}$ and vice versa, replacing $k$ by $n-k$.) We compute $c^{-m-1}\left(\beta^{\prime}\right)=-\alpha_{\ell-m}-\cdots-\alpha_{k}-\alpha_{n}$. Since $\delta$ is fixed by $c$, we see that $\left[c^{-m-1}(\beta): \alpha_{\ell-m}\right]=0$. Each of the $\operatorname{roots} c^{-i}(\beta)$ for $i=0, \ldots, m+1$ is positive, so $\tau_{c}^{-m-1}(\beta)=c^{-m-1}(\beta)$ and thus $\left[\tau_{c}^{-m-1}(\beta): \alpha_{\ell-m}\right]=0$.

Since $\left[\beta: \alpha_{j}\right]=1$, we have $j \in\{\ell+1, \ldots, k\} \cup\{k+m+1, \ldots, n\}$. If $j$ is in $\{\ell+1, \ldots, k\}$, then $\tau_{c}^{-m-1}\left(-\alpha_{j}\right)=\alpha_{j-m}+\cdots+\alpha_{k}+\alpha_{n-m}+\cdots+\alpha_{n}$. If $j$ is in $\{k+m+1, \ldots, n\}$, then $\tau_{c}^{-m-1}\left(-\alpha_{j}\right)=\alpha_{k-m+1}+\cdots+\alpha_{k}+\alpha_{j-m}+\cdots+\alpha_{n}$. Since $\ell<j<k+1$, in either case we have $\left[\tau_{c}^{-m-1}\left(-\alpha_{j}\right): \alpha_{\ell-m}\right]=0$, contradicting condition (2).

Case $B_{n-1}^{(1)}$ : Lemma 2.11 implies that, up to positive scaling, $\phi_{c}$ is $\rho_{n}+\rho_{n-1}-2 \rho_{1}$. Recalling that $\rho_{i}$ is in the basis dual to the simple co-roots and writing $\rho_{i}^{\vee}$ for elements of the basis dual to the simple roots, we have $\rho_{i}^{\vee}=d_{i}^{-1} \rho_{i}$, where the $d_{i}$ are the symmetrizing constants described in Section 2.1. We can take $d_{1}=\frac{1}{2}$ and $d_{i}=1$ for $i \neq 1$, so up to positive scaling $\phi_{c}$ is $\rho_{n}^{\vee}+\rho_{n-1}^{\vee}-\rho_{1}^{\vee}$. Thus we require $\left[\beta: \alpha_{1}\right] \geq\left[\beta: \alpha_{n-1}\right]+\left[\beta: \alpha_{n}\right]$. We have $\delta=2 \sum_{i=1}^{n-2} \alpha_{i}+\alpha_{n-1}+\alpha_{n}$. The positive roots in $\Phi_{\text {fin }}$ are exactly the roots $\sum_{i=\ell}^{m} \alpha_{i}$ for $2 \leq \ell \leq m \leq n-1$ or $2 \sum_{i=1}^{\ell} \alpha_{i}+\sum_{i=\ell+1}^{m} \alpha_{i}$ for $0 \leq \ell<m \leq n-1$.

A positive root $\beta$ with full support, at least one simple-root coordinate $=1$ and $\left[\beta: \alpha_{1}\right] \geq\left[\beta: \alpha_{n-1}\right]+\left[\beta: \alpha_{n}\right]$ is of one of the following forms:

(a) $\beta^{\prime}+\delta$ for $\beta^{\prime} \in \Phi_{\text {fin }}^{+}$with $\left[\beta^{\prime}: \alpha_{1}\right] \geq\left[\beta^{\prime}: \alpha_{n-1}\right]$.

(b) $-\beta^{\prime}+\delta$ for $\beta^{\prime}=\sum_{i=\ell}^{m} \alpha_{i}$ for $2 \leq \ell \leq m \leq n-2$.

(c) $-\beta^{\prime}+2 \delta$ for $\beta^{\prime} \in \Phi_{\text {fin }}^{+}$with $\left[\beta^{\prime}: \alpha_{n-1}\right]=1$ and $\left[\beta^{\prime}: \alpha_{1}\right] \leq 1$.

If $\beta$ is of form (a) and $\beta^{\prime} \in \Phi_{\{2, \ldots, n-2\}}^{+}$, then $\beta$ is in $U^{c}$ and has component-full tube support, so we can assume that either $\left[\beta^{\prime}: \alpha_{1}\right]$ or $\left[\beta^{\prime}: \alpha_{n-1}\right]$ is positive. But since $\left[\beta^{\prime}: \alpha_{1}\right] \geq\left[\beta^{\prime}: \alpha_{n-1}\right]$, we have $\left[\beta^{\prime}: \alpha_{1}\right] \geq 1$. Thus $\beta^{\prime}$ is of the form $2 \sum_{i=1}^{\ell} \alpha_{i}+\sum_{i=\ell+1}^{m} \alpha_{i}$ for $0 \leq \ell<m \leq n-1$. If $\beta^{\prime}$ is $\sum_{i=1}^{n-1} \alpha_{i}$ (that is, if $\ell=0$ and $m=$ $n-1)$, then $\beta^{\prime}=\beta_{n-2}$ in Table 2.1, so again $\beta$ is in $U^{c}$ and has component-full tube support, and we rule out his case. If $\ell=0$ and $m<n-1$, then $c^{-m}\left(\beta^{\prime}\right)=-\sum_{i=1}^{n} \alpha_{i}$, and thus $c^{-m}(\beta)=c^{-m}\left(\beta^{\prime}\right)+\delta=\sum_{i=1}^{n-2} \alpha_{i}$, Since all these roots are positive, we have $\tau_{c}^{-m}(\beta)=\sum_{i=1}^{n-2} \alpha_{i}$. We have $j=n-1$ or $j=n$. In either case, we calculate $\tau_{c}^{-m}\left(\alpha_{j}\right)$ and find that either its $\alpha_{n-1}$ - or $\alpha_{n}$-coefficient is zero. Similarly, if $\ell>0$, we compute $c^{-\ell}\left(\beta^{\prime}\right)=-\sum_{i=m-\ell+1}^{n} \alpha_{i}$ and see that $\tau_{c}^{-\ell}=c^{-\ell}(\beta)$ has both its $\alpha_{n-1}$-coefficient and its $\alpha_{n}$-coefficient zero, while $\tau_{c}^{-\ell}\left(\alpha_{j}\right)$ has either its $\alpha_{n-1^{-}}$or $\alpha_{n}$-coefficient zero. In either case, we have found a contradiction to (2).

If $\beta$ is of form (b), then $\beta$ is in $U^{c}$ and has component-full tube support. 
Finally, if $\beta$ is of form (c), then $\beta^{\prime}=\sum_{i=\ell}^{n-1}$ for some $1 \leq \ell \leq n-1$. If $\ell=1$, then $\beta$ is in $U^{c}$ and has component-full tube support, so we assume $\ell>1$. We compute $c^{-\ell+1}\left(\beta^{\prime}\right)=\delta+\alpha_{q}$, where $q$ is $n$ if $\ell$ is even or $q$ is $n-1$ if $\ell$ is odd. Therefore $c^{-\ell+1}(\beta)=\delta-\alpha_{q}$, and since all these roots are positive, we have $\tau_{c}^{-\ell+1}(\beta)=\delta-\alpha_{q}$, which has $\alpha_{q}$-coefficient zero. In this case, $j=n-1$ and we calculate $\tau_{c}^{-\ell+1}\left(-\alpha_{j}\right)=$ $\sum_{i=n-\ell+1}^{n_{2}} \alpha_{i}+\alpha_{p}$, where $p$ is $n-1$ if $\ell$ is even or $p$ is $n$ if $\ell$ is odd. Both have $\alpha_{q}$-coefficient zero, so we have again contradicted (2).

Case $C_{n-1}^{(1)}$ : Lemma 2.11 implies that, up to positive scaling, $\phi_{c}$ is $\rho_{n}-\rho_{1}$. Since the symmetrizing constants $d_{1}$ and $d_{n}$ are the same, up to scaling this is $\rho_{n}^{\vee}-\rho_{1}^{\vee}$, so we require $\left[\beta: \alpha_{1}\right] \geq\left[\beta: \alpha_{n}\right]$. We have $\delta=\sum_{i=1}^{n} \alpha_{i}+\sum_{i=2}^{n-1} \alpha_{i}$. The positive roots in $\Phi_{\text {fin }}$ are exactly the roots $\sum_{i=\ell}^{m} \alpha_{i}$ for $2 \leq \ell \leq m \leq n-1$ or $\sum_{i=1}^{m} \alpha_{i}+\sum_{i=2}^{\ell} \alpha_{i}$ for $1 \leq \ell \leq m \leq n-1$.

A positive root $\beta$ with full support, at least one simple-root coordinate $=1$ and $\left[\beta: \alpha_{1}\right] \geq\left[\beta: \alpha_{n}\right]$ is either $\beta^{\prime}+\delta$ for $\beta^{\prime} \in \Phi_{\text {fin }}^{+}$or $-\beta^{\prime}+\delta$ for $\beta^{\prime} \in \Phi_{\{2, \ldots, n-1\}}^{+}$. First, assume $\beta=\beta^{\prime}+\delta$ for $\beta^{\prime} \in \Phi_{\text {fin }}^{+}$. If $\beta^{\prime} \in \Phi_{\{2, \ldots, n-1\}}$, then $\beta \in U^{c}$ and $\operatorname{Supp}_{\Xi}(\beta)$ is component-full, so we assume that $\beta^{\prime}=\sum_{i=1}^{m} \alpha_{i}+\sum_{i=2}^{\ell} \alpha_{i}$ for $1 \leq \ell \leq m \leq n-1$. We calculate $c^{-m}\left(\beta^{\prime}\right)=-\sum_{i=1}^{n} \alpha_{i}$, so that $c^{-m}(\beta)=\delta-\sum_{i=1}^{n} \alpha_{i}=\sum_{i=2}^{n-1} \alpha_{i}$. These roots are all positive, so $\tau_{c}^{-m}(\beta)=c^{-m}(\beta)$, which has $\alpha_{1}$-coordinate zero. In this case, $j=n$, so we compute $\tau_{c}^{-m}\left(-\alpha_{j}\right)=2 \sum_{i=n-m+1}^{n-1}+\alpha_{n}$, which also has $\alpha_{1}$-coordinate zero since $m \leq n-1$. We have a contradiction to $(2)$ in this case.

Next, assume $\beta=-\beta^{\prime}+\delta$ for $\beta^{\prime} \in \Phi_{\{2, \ldots, n-1\}}^{+}$. Specifically, $\beta^{\prime}=\sum_{i=\ell}^{m} \alpha_{i}$ for $2 \leq \ell \leq m \leq n-1$. We compute $c^{-\ell+1}\left(\beta^{\prime}\right)=\sum_{i=1}^{n} \alpha_{i}+\sum_{i=2}^{m-\ell+1} \alpha_{i}$, so $\tau_{c}^{-\ell+1}(\beta)=$ $c^{-\ell+1}(\beta)=\sum_{i=m-\ell+2}^{n-1} \alpha_{i}$, which has $\alpha_{1^{-}}$and $\alpha_{n}$-coordinates zero. In this case, either $j \in\{1, n\}$ or $j \in\{\ell, \ell+1, \ldots, m\}$. If $j=n$, then $\tau_{c}^{-\ell+1}\left(-\alpha_{j}\right)=2 \sum_{i=n-\ell+2}^{n-1}+\alpha_{n}$, which also has $\alpha_{1}$-coordinate zero. If $j \in\{\ell, \ldots, m\}$, then $\tau_{c}^{-1}\left(-\alpha_{j}\right)=\sum_{i=j}^{n} \alpha_{i}$, so $\tau_{c}^{-\ell+1}\left(-\alpha_{j}\right)=\sum_{i=j-\ell+2}^{n} \alpha_{i}+\sum_{i=n-\ell+2}^{n-1} \alpha_{i}$, which also has $\alpha_{1}$-coordinate zero. We have contradicted (2) except when $j=1$. In that final case, we compute $\tau_{c}^{n-\ell}(\beta)=$ $\sum_{i=m-\ell+2}^{n-1} \alpha_{i}$ and $\tau_{c}^{n-\ell}\left(-\alpha_{1}\right)=\alpha_{1}+2 \sum_{i=2}^{n-\ell} \alpha_{i}$, and we have contradicted (2) in this final case.

Case $D_{n-1}^{(1)}$ : By Lemma 2.11, up to positive scaling $\phi_{c}$ is $\rho_{n}+\rho_{n-1}-\rho_{2}-\rho_{1}$, so we want $\beta$ with $\left[\beta: \alpha_{1}\right]+\left[\beta: \alpha_{2}\right] \geq\left[\beta: \alpha_{n-1}\right]+\left[\beta: \alpha_{n}\right]$. We have $\delta=\sum_{i=1}^{n} \alpha_{i}+\sum_{i=3}^{n-2} \alpha_{i}$. Each positive root in $\Phi_{\text {fin }}$ is either a sum of adjacent roots, with coefficients 1 , along a path in the diagram for $\Phi_{\text {fin }}$ or is of the form $\sum_{i=1}^{m} \alpha_{i}+\sum_{i=3}^{\ell} \alpha_{i}$ for some $\ell$ and $m$ with $2 \leq \ell<m \leq n-1$.

A positive root $\beta$ with full support, at least one simple-root coordinate $=1$ and $\left[\beta: \alpha_{1}\right]+\left[\beta: \alpha_{2}\right] \geq\left[\beta: \alpha_{n-1}\right]+\left[\beta: \alpha_{n}\right]$ is of one of the following three forms:

(a) $\beta^{\prime}+\delta$ for $\beta^{\prime} \in \Phi_{\text {fin }}^{+}$with $\left[\beta^{\prime}: \alpha_{1}\right]+\left[\beta^{\prime}: \alpha_{2}\right] \geq\left[\beta^{\prime}: \alpha_{n-1}\right]$.

(b) $-\beta^{\prime}+\delta$ for $\beta^{\prime} \in \Phi_{\{3, \ldots, n-2\}}^{+}$.

(c) $-\beta^{\prime}+2 \delta$ for $\beta^{\prime} \in \Phi_{\text {fin }}^{+}$with $\left[\beta^{\prime}: \alpha_{n-1}\right]=1$ and $\left[\beta^{\prime}: \alpha_{1}\right]+\left[\beta^{\prime}: \alpha_{2}\right] \leq 1$.

If $\beta$ is of form (a) and $\beta^{\prime} \in \Phi_{\{3, \ldots, n-2\}}$, then $\beta \in U^{c}$ and $\operatorname{Supp}_{\Xi}(\beta)$ is componentfull, so we can rule out this case. Thus at least one of $\left[\beta^{\prime}: \alpha_{1}\right],\left[\beta^{\prime}: \alpha_{2}\right]$ and $\left[\beta^{\prime}: \alpha_{n-1}\right]$ is 1 , but since $\left[\beta^{\prime}: \alpha_{1}\right]+\left[\beta^{\prime}: \alpha_{2}\right] \geq\left[\beta^{\prime}: \alpha_{n-1}\right]$, we see that at least one of $\left[\beta^{\prime}: \alpha_{1}\right]$ and $\left[\beta^{\prime}: \alpha_{2}\right]$ is 1 . By symmetry, we can assume $\left[\beta^{\prime}: \alpha_{1}\right]=1$. If also $\left[\beta^{\prime}: \alpha_{n-1}\right]=1$ but $\left[\beta^{\prime}: \alpha_{2}\right]=0$, then again $\beta \in U^{c}$ and $\operatorname{Supp}_{\Xi}(\beta)$ is componentfull. Thus we need to consider two cases: $\beta^{\prime}=\alpha_{1}+\sum_{i=3}^{\ell} \alpha_{i}$ for $2 \leq \ell \leq n-2$ or $\left[\beta^{\prime}: \alpha_{1}\right]=\left[\beta^{\prime}: \alpha_{2}\right]=1$. 
First, take $\beta^{\prime}=\alpha_{1}+\sum_{i=3}^{\ell} \alpha_{i}$ for $2 \leq \ell \leq n-2$. In this case, $j \in\{2, n-1, n\}$. If $\ell=2$, so that $\beta^{\prime}=\alpha_{1}$, then $c^{-1}\left(\beta^{\prime}\right)=-\alpha_{1}-\sum_{i=3}^{n} \alpha_{i}$, so $\left[c^{-1}(\beta): \alpha_{1}\right]=0$. Since $\beta$ and $c^{-1}(\beta)$ are positive, $\tau_{c}^{-1}(\beta)=c(\beta)$, so $\left[\tau_{c}^{-1}(\beta): \alpha_{1}\right]=0$. But also $\left[\tau_{c}\left(-\alpha_{j}\right): \alpha_{1}\right]=0$ for $j \in\{2, n-1, n-2\}$, so condition (2) fails. Set $p=1$ if $\ell$ is even or $p=2$ if $\ell$ is odd and define $q$ such that $\{p, q\}=\{1,2\}$. If $3 \leq \ell \leq n-2$, then we calculate $\tau_{c}^{-\ell+1}(\beta)=\alpha_{q}+\sum_{i=3}^{n-2} \alpha_{i}$. Meanwhile, for $j \in\{n-1, n\}, \tau_{c}^{-\ell+1}\left(-\alpha_{j}\right)$ has positive coordinates only at indices $\geq n-\ell+1 \geq 3$. Thus $\operatorname{Supp}_{\Pi}\left(\tau_{c}^{-\ell+1}\left(\alpha_{j}\right), \tau_{c}^{-\ell+1}(\beta)\right)$ does not contain $\alpha_{p}$, so condition (2) fails for $j \in\{n-1, n\}$. Also, $\tau_{c}^{-1}\left(-\alpha_{2}\right)=\sum_{i=2}^{n} \alpha_{i}$, so $\tau_{c}^{-\ell+1}\left(-\alpha_{2}\right)$ has $\alpha_{p}$-coordinate zero, and condition (2) fails for $j=2$ as well.

Next suppose $\left[\beta^{\prime}: \alpha_{1}\right]=\left[\beta^{\prime}: \alpha_{2}\right]=1$. If $\left[\beta^{\prime}: \alpha_{n-1}\right]=1$, then $j=n$ and $\beta^{\prime}=$ $\sum_{i=1}^{n-1} \alpha_{i}+\sum_{i=3}^{\ell} \alpha_{i}$ for some $\ell$ with $2 \leq \ell \leq n-2$. Now set $p=n-1$ if $\ell$ is even or $p=n$ if $\ell$ is odd and define $q$ such that $\{p, q\}=\{n-1, n\}$. We compute $\tau_{c}^{-\ell+1}(\beta)=\delta-\alpha_{p}$ and $\tau_{c}^{-\ell+1}\left(-\alpha_{j}\right)=\sum_{n-\ell+1}^{n-2} \alpha_{i}+\alpha_{q}$, and we have again contradicted condition (2). If $\left[\beta^{\prime}: \alpha_{n-1}\right]=0$, then $j \in\{n-1, n\}$. We compute $\tau_{c}^{-\ell+1}(\beta)=c^{-\ell+1}(\beta)=\delta-c^{-\ell+1}\left(\beta^{\prime}\right)$, which has $\alpha_{n-1^{-}}$and $\alpha_{n^{-}}$coordinates zero. We already saw that $\left[\tau_{c}^{-\ell+1}\left(-\alpha_{n}\right): \alpha_{p}\right]=$ 0 , and by symmetry, we conclude that $\left[\tau_{c}^{-\ell+1}\left(-\alpha_{n-1}\right): \alpha_{q}\right]=0$. Thus whether $j$ is $n$ or $n-1$, we have once gain contradicted condition (2).

If $\beta$ is of form (b), then either $j \in\{1,2, n-1, n\}$ or $j \in \operatorname{Supp}_{\Pi}\left(\beta^{\prime}\right)$. Write $\beta^{\prime}=\sum_{i=\ell}^{m} \alpha_{i}$ with $3 \leq \ell \leq j \leq m \leq n-2$. We compute that $\left[\tau_{c}^{n-m-1}(\beta): \alpha_{n-1}\right]=$ $\left[\tau_{c}^{n-m-1}(\beta): \alpha_{n}\right]=0$. If $j \in\{1,2, n-1, n\}$, then up to the symmetry of the diagram and replacing $c$ by $c^{-1}$, we can take $j=1$. (We have $\left[\beta: \alpha_{1}\right]+\left[\beta: \alpha_{2}\right]=[\beta$ : $\left.\alpha_{n-1}\right]+\left[\beta: \alpha_{n}\right]$ in this case, so symmetry is not broken by the inequality in condition (3).) We compute that $\tau_{c}^{n-m-1}\left(-\alpha_{j}\right)$ is a sum of roots with indices $\leq n-m \leq n-3$. If $j \in \operatorname{Supp}_{\Pi}\left(\beta^{\prime}\right)$, we compute that $\tau_{c}^{n-m-1}\left(-\alpha_{j}\right)$ is a sum of roots with indices $\leq j+n-m-2 \leq n-2$. In either case, we have contradicted condition (2).

If $\beta$ is of form (c) and $\left[\beta^{\prime}: \alpha_{1}\right]+\left[\beta^{\prime}: \alpha_{2}\right]=1$, then $\beta^{\prime}$ is either $\beta_{n-3}$ or $\beta_{n-2}$ in the notation of Table 2.1. In either case, $\beta$ is in $U^{c}$ and $\operatorname{Supp}_{\Xi}(\beta)$ is component-full. Thus we can take $\beta^{\prime}=\sum_{i=\ell}^{n-1} \alpha_{i}$ for $3 \leq \ell \leq n-1$. In this case, $j$ must be $n-1$. We compute $\tau_{c}^{\ell+2}(\beta)=\delta-\alpha_{q}$, so that in particular $\left[\tau_{c}^{-\ell+2}(\beta): \alpha_{q}\right]=0$. Recalling that $j=n-1$, we calculate $\tau_{c}^{-\ell+2}\left(-\alpha_{j}\right)=\sum_{i=n-\ell+2}^{n-2} \alpha_{i}+\alpha_{p}$, contradicting condition (2).

Proof of Proposition 7.11. We check the proposition type-by-type using the choice of $c$ and the determination of the simple roots of $\Upsilon_{\text {fin }}^{c}$ given in Table 2.1. We continue the notation from the proof of Lemma 5.8 and index the components of $\Upsilon^{c}$ by indices $i$, writing $\beta_{\text {aff }}^{i}$ for the unique simple root in the $i^{\text {th }}$ component that is not in $\Phi_{\text {fin. }}$. Simple-root coordinates of the roots $\beta_{\text {aff }}^{i}$ can be computed using simple-root coordinates of $\delta$ found, for example, in [24, Table Aff 1].

Case $A_{n-1}^{(1)}$ : Diagram automorphisms act transitively on the simple roots.

Case $B_{n-1}^{(1)}$ : We need to show that $j \in\{n-1, n\}$. If $\beta_{n-2} \in R$, then $j$ can't be in $\{1, \ldots, n-1\}$, so $j=n$. Otherwise $\beta_{\text {aff }}^{2}=\alpha_{n}+\sum_{i=1}^{n-2} \alpha_{i}$ is in $R$, so $j=n-1$.

Case $C_{n-1}^{(1)}$ : We need $j \in\{1, n\}$. If $j \in\{2, \ldots, n-1\}$, then $\beta_{j-1} \notin R$, so $\beta_{\text {aff }}^{1}=$ $\sum_{i=1}^{n} \alpha_{i} \in R$, and this is a contradiction.

Case $D_{n-1}^{(1)}$ : We need $j \in\{1,2, n-1, n\}$. If $\beta_{n-3} \in R$, then $j \in\{2, n\}$. Otherwise, $\beta_{\text {aff }}^{2}=\delta-\beta_{n-3}=\alpha_{n}+\sum_{i=2}^{n-2} \in R$, so $j \in\{1, n-1\}$.

Case $E_{6}^{(1)}$ : We need $j \in\{1,3,7\}$. If $\beta_{5} \in R$, then $j \in\{1,3,7\}$. Otherwise, $\beta_{\text {aff }}^{3}=$ $\delta-\beta_{5} \in R$, and there is no possible $j$. 
Case $E_{7}^{(1)}:$ We need $j \in\{2,8\}$. If $\beta_{6} \in R$, then $j \in\{2,8\}$. Otherwise, $\beta_{\text {aff }}^{3} \in R$, and there is no possible $j$.

Case $E_{8}^{(1)}:$ We need $j=9$. If $\beta_{7} \in R$, then $j=9$. Otherwise $\beta_{\text {aff }}^{3} \in R$, and there is no possible $j$.

Case $F_{4}^{(1)}$ : We need $j=5$. If $\beta_{2} \in R$, then $j=5$. Otherwise, $\beta_{\text {aff }}^{1} \in R$, and there is no possible $j$.

Case $G_{2}^{(1)}$ : We need $j=3$, which is forced if $\beta_{1} \in R$. Otherwise $\beta_{\text {aff }}^{1} \in R$, and there is no possible $j$.

\section{Connections with Cluster algebras: g-Vectors and d-Vectors}

In this section, we connect the real $c$-cluster fan $\operatorname{Fan}_{c}^{\mathrm{re}}(\Phi)$ to the g-vector fan and the $\mathbf{d}$-vector fan of the corresponding cluster algebra by proving Theorems 1.1 and 1.2. We also discuss evidence for Conjecture 1.3.

9.1. Cluster algebras notation and conventions. We begin by reviewing notation and establishing conventions. We assume the basic definitions (from [19]) of exchange matrices, mutations, cluster variables, clusters of cluster variables, seeds and the d-vector or denominator vector and g-vector of a cluster variable.

We are interested in acyclic exchange matrices whose underlying Cartan matrix $A$ is of affine type. In this case, the exchange matrix $B$ is of affine type in the sense discussed in the introduction. Let $\Phi$ be the root system defined by the Cartan matrix $A$, and continue the notation of the rest of the paper for root systems. Since $B$ is acyclic, we can associate to it the Coxeter element $c$ obtained as the product of the simple reflections $S$ ordered so that $s_{i}$ precedes $s_{j}$ whenever $b_{i j}>0$. We assume that $B$ is indexed so that $b_{i j} \geq 0$ whenever $i<j$; with this convention $c$ can be written $s_{1} \cdots s_{n}$ as in the rest of the paper. The notation $\mathcal{A}_{\Sigma}$ stands for the cluster algebra determined by a seed $\Sigma$ and $\mathcal{A}_{\bullet}(B)$ stands for the principalcoefficients cluster algebra determined by $B$.

We write $\mathbf{d}(x)$ or $\mathbf{d}_{\Sigma}(x)$ for the denominator vector of a cluster variable $x$ with respect to the seed $\Sigma$ and similarly $\mathbf{g}(x)$ or $\mathbf{g}_{\Sigma}(x)$ for $\mathbf{g}$-vectors. In Theorem 1.2, $\mathbf{d}$-vectors are written as vectors in the root lattice: Specifically, if $x$ has denominator

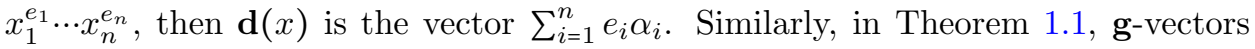
are written as vectors in the weight lattice, and the realization of the $\mathbf{g}$-vector as an integer vector is obtained by taking fundamental-weight coordinates.

For each cluster in $\mathcal{A}_{\bullet}(B)$, the nonnegative linear span of the $\mathbf{g}$-vectors in the cluster is a full-dimensional simplicial cone, and these cones, together, form the $\mathbf{g}$-vector fan. The map from cluster variables to $\mathbf{g}$-vectors (or to rays in the $\mathbf{g}$-vector fan) is a bijection, and the map from clusters to maximal cones in the g-vector fan is also a bijection. Indeed, the simplicial complex underlying the g-vector fan is isomorphic to the cluster complex. These facts about $\mathbf{g}$-vector fans have been proved in various special cases before being proved in general in [20]. In particular, they were proved in $[31,34,35]$ in finite and affine type using the combinatorics of root systems and Coxeter groups.

We define a piecewise linear map $\nu_{c}: V \rightarrow V^{*}$. A similar linear map, also called $\nu_{c}$, was defined in [34, Section 5.3], but a piecewise-linear version is more useful here. The two maps agree on the nonnegative span of the simple roots and were only applied to positive roots in [34]. Suppose $\beta \in V$ and $I \subseteq\{1, \ldots, n\}$ is the set of indices $i$ such that $\left[\beta: \alpha_{i}\right]<0$. We write $\beta_{+}$for the vector $\beta-\sum_{i \in I}\left[\beta: \alpha_{i}\right] \alpha_{i}$ and 
define

$$
\nu_{c}(\beta)=-\sum_{i \in I}\left[\beta: \alpha_{i}\right] \rho_{i}-\sum_{i \notin I} E_{c}\left(\alpha_{i}^{\vee}, \beta_{+}\right) \rho_{i},
$$

where $E_{c}$ is as defined in (2.1). The linear version of $\nu_{c}$ has an inverse defined in [34, Section 3.3]. Using the same construction on each orthant, an inverse to the piecewise-linear map $\nu_{c}$ is easily constructed, and we see that $\nu_{c}$ is a piecewise-linear homeomorphism from $V$ to $V^{*}$.

9.2. Proofs of Theorems $\mathbf{1 . 1}$ and 1.2. As a first step to proving Theorems 1.1 and 1.2, we recall from [35] the construction of the $\mathbf{g}$-vector fan as the doubled Cambrian fan, defined in terms of sortable elements. We will be as brief as possible, skipping much of the combinatorics and geometry of sortable elements and Cambrian fans.

Let $c$ be a Coxeter element of a Coxeter group $W$. The $c$-sortable elements of $W$ can be characterized by the following recursion, together with the base condition that the identity element is $c$-sortable in any Coxeter group and for any $c$. Suppose $w \in W$ and $s$ is initial in $c$.

- If $s \nless w$, then $w$ is $c$-sortable if and only if it is contained in the parabolic subgroup generated by $S \backslash\{s\}$ and is $s c$-sortable as an element of that subgroup.

- If $s \leq w$, then $w$ is $c$ sortable if and only if $s w$ is $s c s$-sortable.

Here the relation $\leq$ refers to the weak order on $W$, and the condition $s \leq w$ is equivalent to the condition that $w$ admits a reduced word whose first letter is $s$.

We define recursively a map $\mathrm{cl}_{c}$ from $c$-sortable elements to roots. The recursion was originally [29, Lemma 8.5], but can be taken as a definition of the map. Let $w$ be $c$-sortable and suppose $s$ is initial in $c$.

- If $w$ is the identity, then $\operatorname{cl}_{c}(w)=-\Pi$.

- If $s \not w$ then $\mathrm{cl}_{c}(w)=\left\{-\alpha_{s}\right\} \cup \mathrm{cl}_{s c}(w)$.

- If $s \leq w$ then $\operatorname{cl}_{c}(w)=\sigma_{s}\left(\mathrm{cl}_{s c s}(s w)\right)$.

For each $c$-sortable element, we define $\operatorname{Cone}_{c}(v)$ to be the nonnegative linear span of $\nu_{c}\left(\mathrm{cl}_{c}(v)\right)$. This definition is equivalent to the definition in [34, Section 5.2] in light of [34, Theorem 5.35]. The c-Cambrian fan $\mathcal{F}_{c}$ is the collection of all cones $\operatorname{Cone}_{c}(v)$ for $c$-sortable elements $v$, and all faces of these cones. The doubled c-Cambrian fan $\mathcal{D} \mathcal{F}_{c}$ is the collection consisting of the cones in $\mathcal{F}_{c}$ and the negations of cones in the $c^{-1}$-Cambrian fan $\mathcal{F}_{c^{-1}}$. That is, $\mathcal{D} \mathcal{F}_{c}=\mathcal{F}_{c} \cup\left(-\mathcal{F}_{c^{-1}}\right)$.

The following theorem is [35, Corollary 1.3].

Theorem 9.1. Suppose $B$ is an acyclic exchange matrix whose associated Cartan matrix is of affine type and whose associated Coxeter element is $c$. Then the doubled c-Cambrian fan $\mathcal{D} \mathcal{F}_{c}$ coincides with the $\mathbf{g}$-vector fan for the cluster algebra $\mathcal{A}_{\bullet}(B)$.

We also need the following weak version of [35, Corollary 4.9], where $\left|\mathcal{D} \mathcal{F}_{c}\right|$ denotes the union of the cones in $\mathcal{D} \mathcal{F}_{c}$.

Theorem 9.2. $V^{*} \backslash\left|\mathcal{D} \mathcal{F}_{c}\right|$ is an $(n-1)$-dimensional relatively open cone.

The final ingredient needed for the proof of Theorem 1.1 is the following lemma.

Lemma 9.3. The map $\beta \mapsto \nu_{c^{-1}}^{-1}\left(-\nu_{c}(\beta)\right)$, applied to $\Phi_{c}$, coincides with $\tau_{c}$. 
Proof. We again think of $E_{c}$ as the $n \times n$ matrix whose $i j$-entry is $a_{i j}$ if $i>j$, is 1 if $i=j$, and is 0 if $i<j$. But this time, we think of that matrix as taking the simple root coordinates of a vector in $V$ to the fundamental weight coordinates of a vector in $V^{*}$. In particular, $E_{c}$ is the matrix of $\nu_{c}$ when the latter is applied to positive roots. If $\beta$ is neither of the form $-\alpha_{j}$ nor of the form $s_{n} \cdots s_{j+1} \alpha_{j}$, then Proposition 3.12(2) implies that $\tau_{c}$ acts as $c$ on $\beta$ and that both $\beta$ and $\tau_{c} \beta$ are positive roots. Thus $\nu_{c}$ acts on $\beta$ by the matrix $E_{c}$ and $\nu_{c^{-1}}$ acts on $\tau_{c} \beta=c \beta$ by the matrix $E_{c^{-1}}$, and we see by Theorem 2.6 that $-\nu_{c} \beta=\nu_{c^{-1}} \tau_{c} \beta$.

If $\beta=-\alpha_{j}$ for some $j$, then $-\nu_{c}(\beta)=-\rho_{j}$ and $\nu_{c^{-1}}\left(\tau_{c} \beta\right)=\nu_{c^{-1}}\left(s_{1} \cdots s_{j-1} \alpha_{j}\right)$. A simple calculation (see for example [32, Lemma 2.9]) shows that

$$
s_{1} \cdots s_{j-1} \alpha_{j}=\sum_{1 \leq i_{1}<i_{2}<\cdots<i_{k}=j}\left(-a_{i_{1} i_{2}}\right)\left(-a_{i_{2} i_{3}}\right) \cdots\left(-a_{i_{k-1} i_{k}}\right) \alpha_{i_{1}},
$$

with $k$ varying from 1 to $j$. Thus $\nu_{c^{-1}}\left(s_{1} \cdots s_{j-1} \alpha_{j}\right)$ is

$$
-\sum_{i=1}^{n} E_{c^{-1}}\left(\alpha_{i}^{\vee}, \sum_{1 \leq i_{1}<i_{2}<\cdots<i_{k}=j}\left(-a_{i_{1} i_{2}}\right)\left(-a_{i_{2} i_{3}}\right) \cdots\left(-a_{i_{k-1} i_{k}}\right) \alpha_{i_{1}}\right) \rho_{i} .
$$

This sum has nonzero terms for $i \leq i_{1}$. Separating out the terms for $i=i_{1}$, the sum becomes

$$
\begin{aligned}
-\sum_{1 \leq i_{1}<i_{2}<\cdots<i_{k}=j}\left(-a_{i_{1} i_{2}}\right)\left(-a_{i_{2} i_{3}}\right) \cdots\left(-a_{i_{k-1} i_{k}}\right) \rho_{i_{1}} \\
-\quad \sum_{1 \leq i<i_{1}<i_{2}<\cdots<i_{k}=j} a_{i i_{1}}\left(-a_{i_{1} i_{2}}\right)\left(-a_{i_{2} i_{3}}\right) \cdots\left(-a_{i_{k-1} i_{k}}\right) \rho_{i} .
\end{aligned}
$$

The terms in the two sums cancel each other out, except for the $k=1$ term in the first sum, so the expression for $\nu_{c^{-1}}\left(s_{1} \cdots s_{j-1} \alpha_{j}\right)$ collapses to $-\rho_{j}$ as desired.

If $\beta=s_{n} \cdots s_{j+1} \alpha_{j}$ for some $j$, then $\nu_{c^{-1}}\left(\tau_{c} \beta\right)=\nu_{c^{-1}}\left(-\alpha_{j}\right)=\rho_{j}$. Replacing $c^{-1}$ by $c$ in the argument above, one sees that $-\nu_{c}(\beta)$ is $\rho_{j}$ as well.

Proof of Theorem 1.1. In light of Theorem 9.1, to prove the first assertion of Theorem 1.1, we show that $\nu_{c}$ induces an isomorphism from $\operatorname{Fan}_{c}^{\mathrm{re}}(\Phi)$ to $\mathcal{D} \mathcal{F}_{c}$.

We first show that for every $c$-sortable element $v$, the $\operatorname{set}_{c l}(v)$ is a real $c$-cluster. The simple argument is follows the first paragraph of the proof of the finite-type result [29, Theorem 8.1], except that instead appealing to induction on rank, we appeal to the finite-type result. We do, however, argue by induction on the length of $v$. Writing $c=s_{1} \cdots s_{n}$, if $s_{1} \nless v$, then $v$ is $s_{1} c$-sortable in the parabolic subgroup generated by $\left\{s_{2}, \ldots, s_{n}\right\}$. By the finite-type result, $\operatorname{cl}_{s_{1} c}(v)$ is an $s_{1} c$-cluster, so Proposition 5.4(3) says that $\mathrm{cl}_{c}(v)$ is a $c$-cluster. If $s_{1} \leq v$, then the length of $s_{1} v$ is shorter than the length of $v$, so by induction $\mathrm{cl}_{s_{1} c s_{1}}\left(s_{1} v\right)$ is an $s_{1} c s_{1}$-cluster, and therefore $\mathrm{cl}_{c}(v)$ is a $c$-cluster by Proposition 5.4(2).

Since $\operatorname{Cone}_{c}(v)$ is the nonnegative linear span of $\nu_{c}\left(\mathrm{cl}_{c}(v)\right)$ for any $c$-sortable element $v$, we conclude that every cone in the $c$-Cambrian fan $\mathcal{F}_{c}$ is the image, under $\nu_{c}$, of a cone in $\operatorname{Fan}_{c}^{\mathrm{re}}(\Phi)$.

We similarly want to show that every cone in $-\mathcal{F}_{c^{-1}}$ is the image, under $\nu_{c}$, of a cone in $\operatorname{Fan}_{c}^{\mathrm{re}}(\Phi)$. The cones in $-\mathcal{F}_{c^{-1}}$ are the nonnegative linear spans of $-\nu_{c^{-1}}\left(\mathrm{cl}_{c^{-1}}(v)\right)$ for $c^{-1}$-sortable elements $v$. Thus we want to show that the set $\nu_{c}^{-1}\left(-\nu_{c^{-1}}\left(\mathrm{cl}_{c^{-1}}(v)\right)\right)$ is a $c$-cluster for all $c^{-1}$-sortable elements $v$. By the argument above, with $c^{-1}$ replacing $c$, we know that $c_{c^{-1}}(v)$ is a $c^{-1}$-cluster. Since $\nu_{c}^{-1}\left(-\nu_{c^{-1}}\left(\operatorname{cl}_{c^{-1}}(v)\right)\right)=\tau_{c^{-1}} \mathrm{cl}_{c^{-1}}(v)$ by Lemma 9.3, Proposition 5.4(1) implies that $\nu_{c}^{-1}\left(-\nu_{c^{-1}}\left(\operatorname{cl}_{c^{-1}}(v)\right)\right)$ is a $c^{-1}$-cluster as well, and thus a $c$-cluster by Proposition 4.10. 
We have showed that $\nu_{c}^{-1}$ maps every cone in $\mathcal{D} \mathcal{F}_{c}$ to a cone in $\operatorname{Fan}_{c}^{\mathrm{re}}(\Phi)$. Since $\nu_{c}^{-1}: V^{*} \rightarrow V$ is a homeomorphism and since by Theorem 9.2 the complement of $\mathcal{D} \mathcal{F}_{c}$ in $V^{*}$ is an $(n-1)$-dimensional cone, the complement of $\nu_{c}^{-1}\left(\mathcal{D} \mathcal{F}_{c}\right)$ is $(n-1)$ dimensional. In particular, since the maximal cones of $\operatorname{Fan}_{c}^{\text {re }}(\Phi)$ are $n$-dimensional by Proposition 5.14(2), every maximal cone of $\operatorname{Fan}_{c}^{\text {re }}(\Phi)$ is in the image of $\mathcal{D} \mathcal{F}_{c}$ under $\nu_{c}^{-1}$. Thus because $\nu_{c}^{-1}$ is a homeomorphism and because both $\mathcal{D} \mathcal{F}_{c}$ and $\operatorname{Fan}_{c}^{\text {re }}(\Phi)$ are fans, we see that $\nu_{c}^{-1}$ induces an isomorphism from $\mathcal{D} \mathcal{F}_{c}$ to $\operatorname{Fan}_{c}^{\text {re }}(\Phi)$. Equivalently, $\nu_{c}$ induces an isomorphism from $\operatorname{Fan}_{c}^{\mathrm{re}}(\Phi)$ to $\mathcal{D} \mathcal{F}_{c}$.

The second assertion of Theorem 1.1 now follows because, as mentioned above, the map $x \mapsto \mathbf{g}(x)$ is an isomorphism from the cluster complex to the simplicial complex underlying the g-vector fan. The latter isomorphism is proved in affine type using the combinatorics of root systems and Coxeter groups [31, 34, 35], so this entire proof occurs in that combinatorial setting.

To establish Theorem 1.2, we need the following theorem, which was conjectured as [34, Conjecture 3.21] and proved as [38, Proposition 9]. (In fact, [38, Proposition 9] only establishes Theorem 9.4 for non-initial cluster variables, but extending the theorem to include initial cluster variables is easy.)

Theorem 9.4. Suppose $B$ is an acyclic exchange matrix with associated Coxeter element $c$ and $x$ is a cluster variable in the principal-coefficients cluster algebra associated to $B$. Then $\mathbf{g}(x)=\nu_{c}(\mathbf{d}(x))$.

Combining Theorems 1.1 and 9.4 (and observing that every cone in $\operatorname{Fan}_{c}^{\mathrm{re}}(\Phi)$ is contained in a domain of linearity of $\nu_{c}$ ), we obtain the special case of Theorem 1.2 where $\Sigma$ has principal coefficients. The full statement of Theorem 1.2 then holds by the following well-known observation, which is an easy consequence of [19, Theorem 3.7]. (In the lemma, for a (labeled) seed $\Sigma$, the notation $\Sigma_{i}$ denotes the $i^{\text {th }}$ entry in the cluster in $\Sigma$.)

Lemma 9.5. Suppose $\Sigma$ is a seed (with no conditions on coefficients) and suppose $m$ is some sequence of mutations. If $\Sigma^{\prime}$ is any seed (in any cluster algebra) with the same exchange matrix as $\Sigma$, then $\left.\mathbf{d}_{\Sigma}\left([m(\Sigma)]_{i}\right)=\mathbf{d}_{\Sigma^{\prime}}\left(\left[m\left(\Sigma^{\prime}\right)\right]_{i}\right)\right)$.

We have completed the proofs of Theorems 1.1 and 1.2.

9.3. Evidence for Conjecture 1.3. As preparation for discussing evidence, we prove and quote some preliminary results.

By analogy with source-sink moves on Coxeter elements, we define a source-sink mutation of an acyclic seed to be a mutation in an index $k$ such that the entries in the $k$-th colum of the exchange matrix are either all nonnegative or all nonpositive. Source-sink mutations do not change the Cartan matrix underlying $B$. The following result, which lets us apply source-sink mutations to $\Sigma$ in Conjecture 1.3, is [38, Corollary 10]. The theorem holds in general, not just in finite or affine type.

Theorem 9.6. Suppose $\Sigma$ is an acyclic seed and $x$ is a cluster variable in $\mathcal{A}_{\Sigma}$. If $\Sigma^{\prime}$ is obtained from $\Sigma$ by a source-sink mutation corresponding to the source-sink move $c \rightarrow$ scs, then $\mathbf{d}_{\Sigma^{\prime}}(x)=\sigma_{s} \mathbf{d}_{\Sigma}(x)$.

As a consequence of Theorem 9.6, we have the following proposition.

Proposition 9.7. Conjecture 1.3 holds when $\Sigma^{\prime}$ can be obtained from $\Sigma$ by a sequence of source-sink mutations. 
Proof. When $\Sigma^{\prime}=\Sigma$, this is just Theorem 1.2. Thus Theorem 9.6 and (4.7) combine to prove the proposition.

Each acyclic seed $\Sigma$ defines a Cartan matrix $A$ and a Coxeter element $c$. By Lemma 9.5, Conjecture 1.3 holds or fails simultaneously for all $\Sigma$ corresponding to the same $A$ and $c$. In fact, Conjecture 1.3 is preserved under conjugation of $c$ :

Proposition 9.8. Fix a Cartan matrix $A$ of affine type and a Coxeter element $c$. Suppose Conjecture 1.3 holds for a seed $\Sigma$ corresponding to $A$ and $c$. Then Conjecture 1.3 holds when $\Sigma$ corresponds to $A$ and a Coxeter element conjugate to $c$.

Proof. If $\tilde{c}$ is a Coxeter element conjugate to $c$, then Proposition 2.9 implies that there is a sequence of source-sink mutations taking $\Sigma$ to a seed $\tilde{\Sigma}$ corresponding to $A$ and $\tilde{c}$. Theorem 9.6 and (4.7) show that Conjecture 1.3 for $A$ and $c$ implies Conjecture 1.3 for $A$ and $\tilde{c}$.

Using the surfaces model, one can prove Conjecture 1.3 in affine types A and D.

Theorem 9.9. Conjecture 1.3 holds when $B$ is acyclic of type $A_{n-1}^{(1)}$ or $D_{n-1}^{(1)}$.

The proof of Theorem 9.9 proceeds by analyzing the intersection numbers [16, Definition 8.4] of tagged arcs on the annulus and the twice-punctured disk. By Proposition 9.8, we need consider only one triangulation of each surface. We omit the details, but they can be found in early arXiv versions of this paper.

Finally, we offer additional computational evidence. The following proposition simplifies the process. Given $\Sigma^{\prime}$ and $\beta$ as in Conjecture 1.3 , write $\operatorname{dist}\left(\Sigma^{\prime}, \beta\right)$ for the smallest number of mutations needed to take $\Sigma^{\prime}$ to a seed containing $x(\beta)$.

Proposition 9.10. Fix a Cartan matrix $A$ of affine type, a Coxeter element c, and $d \geq 0$. Suppose Conjecture 1.3 has been verified when $\Sigma$ corresponds to $A$ and $c$, the cluster of $\Sigma^{\prime}$ has nonempty intersection with the cluster of $\Sigma$, and $\operatorname{dist}\left(\Sigma^{\prime}, \beta\right) \leq d$. Then Conjecture 1.3 holds when $\Sigma$ corresponds to $A$ and a Coxeter element conjugate to $c$, for arbitrary $\Sigma^{\prime}$ with $\operatorname{dist}\left(\Sigma^{\prime}, \beta\right) \leq d$.

The proof of Proposition 9.10 uses methods similar to other proofs in this section, and we omit the details. Using this proposition, we have checked Conjecture 1.3 for all rank-3 and rank-4 affine types not covered by Proposition 9.7 or Theorem 9.9, whenever $\operatorname{dist}\left(\Sigma^{\prime}, \beta\right) \leq 100$.

INDEX TO NOTATION

\begin{tabular}{|c|c|c|c|c|c|c|c|}
\hline $\operatorname{adj}_{\alpha}(\beta)$ & page 14 & $\mathbf{g}_{\Sigma}(x)$ & page 38 & $V$ & page 5 & $\theta$ & page 8 \\
\hline aff & page 7 & $K$ & page 5 & $V^{*}$ & page 5 & $\Lambda_{c}$ & page 11 \\
\hline $\mathcal{A}_{\Sigma}$ & page 38 & $M_{\Phi}$ & page 20 & $V_{\text {fin }}$ & page 7 & $\Lambda_{c}^{\mathrm{re}}$ & page 11 \\
\hline $\mathcal{A}_{\bullet}(B)$ & page 38 & $Q$ & page 22 & $W$ & page 5 & $\nu_{c}$ & page 39 \\
\hline$c$ & page 5 & $Q_{c}$ & page 23 & $W_{\text {fin }}$ & page 7 & $\Xi^{c}$ & page 9 \\
\hline $\mathrm{cl}_{c}$ & page 39 & $S$ & page 5 & $\alpha_{i}$ & page 5 & $\Xi_{\text {fin }}^{c}$ & page 9 \\
\hline Cone $(C)$ & page 24 & $S_{\text {fin }}$ & page 7 & $\alpha_{i}^{\mathrm{v}}$ & page 5 & $\Pi$ & page 5 \\
\hline $\mathcal{D} \mathcal{F}_{c}$ & page 40 & $s_{\text {aff }}$ & page 7 & $\alpha_{\text {aff }}$ & page 7 & $\Pi^{\vee}$ & page 5 \\
\hline $\mathbf{d}_{\Sigma}(x)$ & page 38 & $s_{i}$ & page 5 & $\gamma_{c}$ & page 8 & $\Pi_{\text {fin }}$ & page 7 \\
\hline$E_{c}$ & page 6 & $t_{\beta}$ & page 5 & $\delta$ & page 8 & $\rho_{i}$ & page 5 \\
\hline $\operatorname{Fan}_{c}(\Phi)$ & page 24 & $\operatorname{Supp}_{\Pi}(\beta)$ & page 12 & $\delta^{\vee}$ & page 8 & $\sigma_{i}$ & page 13 \\
\hline $\operatorname{Fan}_{c}^{\mathrm{re}}(\Phi)$ & page 24 & $\operatorname{Supp}_{\Xi}(\beta)$ & page 12 & $\Delta_{c}$ & page 24 & $\sigma_{s}$ & page 12 \\
\hline $\mathcal{F}_{c}$ & page 39 & $U^{c}$ & page 8 & $\stackrel{\circ}{c}_{c}$ & page 25 & $\tau_{c}$ & page 13 \\
\hline
\end{tabular}




\begin{tabular}{lll|l|ll|ll}
$\Upsilon^{c}$ & page 9 & $\Phi_{\text {fin }}$ & page 7 & $\overleftarrow{\Psi}^{c}$ & page 9 & $\langle\cdot, \cdot\rangle$ & page 5 \\
$\Upsilon_{\text {fin }}^{c}$ & page 9 & $\Phi_{c}$ & page 11 & $\psi_{c ; j}$ & page 9 & $(\alpha \| \beta)_{c}$ & page 15 \\
$\Phi$ & page 5 & $\Phi_{c}^{\text {re }}$ & page 11 & $\psi_{c ; j}^{\leftarrow}$ & page 9 & $(\alpha \leftarrow \beta)_{c}$ & page 14 \\
$\Phi^{+}$ & page 5 & $\phi_{c}$ & page 9 & $\Omega^{c}$ & page 9 & $(\alpha \rightarrow \beta)_{c}$ & page 14 \\
$\Phi^{\vee}$ & page 8 & $\Psi^{c}$ & page 9 & {$\left[v: \alpha_{i}\right]$} & page 5 & $(\alpha \circlearrowright \beta)_{c}$ & page 14 \\
$\Phi^{\text {re }}$ & page 5 & $\vec{\Psi}^{c}$ & page 9 & {$[x]_{+}$} & page 14 & &
\end{tabular}

Acknowledgments. We thank the anonymous referees for many helpful comments. We are also grateful to Giovanni Cerulli-Irelli and Sibylle Schroll for insights on quiver representations.

\section{REFERENCES}

[1] I. N. Bernstein, I. M. Gelfand, and V. A. Ponomarev. Coxeter functors, and Gabriel's theorem. Uspehi Mat. Nauk, 28(2(170)):19-33, 1973.

[2] Anders Björner and Francesco Brenti. Combinatorics of Coxeter groups, volume 231 of Graduate Texts in Mathematics. Springer, New York, 2005.

[3] Aslak Bakke Buan, Robert Marsh, Markus Reineke, Idun Reiten, and Gordana Todorov. Tilting theory and cluster combinatorics. Adv. Math., 204(2):572-618, 2006.

[4] Aslak Bakke Buan and Robert J. Marsh. Denominators in cluster algebras of affine type. $J$. Algebra, 323(8):2083-2102, 2010.

[5] Aslak Bakke Buan, Robert J. Marsh, Idun Reiten, and Gordana Todorov. Clusters and seeds in acyclic cluster algebras. Proc. Amer. Math. Soc., 135(10):3049-3060 (electronic), 2007. With an appendix coauthored in addition by P. Caldero and B. Keller.

[6] Philippe Caldero and Frédéric Chapoton. Cluster algebras as Hall algebras of quiver representations. Comment. Math. Helv., 81(3):595-616, 2006.

[7] Philippe Caldero and Bernhard Keller. From triangulated categories to cluster algebras. II. Ann. Sci. École Norm. Sup. (4), 39(6):983-1009, 2006.

[8] Philippe Caldero and Andrei Zelevinsky. Laurent expansions in cluster algebras via quiver representations. Mosc. Math. J., 6(3):411-429, 587, 2006.

[9] Michael P. Carr and Satyan L. Devadoss. Coxeter complexes and graph-associahedra. Topology Appl., 153(12):2155-2168, 2006.

[10] Cesar Ceballos and Vincent Pilaud. Denominator vectors and compatibility degrees in cluster algebras of finite type. Trans. Amer. Math. Soc., 367(2):1421-1439, 2015.

[11] Frédéric Chapoton, Sergey Fomin, and Andrei Zelevinsky. Polytopal realizations of generalized associahedra. Canad. Math. Bull., 45(4):537-566, 2002. Dedicated to Robert V. Moody.

[12] Vinay V. Deodhar. A note on subgroups generated by reflections in Coxeter groups. Arch. Math. (Basel), 53(6):543-546, 1989.

[13] Vlastimil Dlab and Claus Michael Ringel. Indecomposable representations of graphs and algebras. Mem. Amer. Math. Soc., 6(173):v+57, 1976.

[14] Matthew Dyer. Reflection subgroups of Coxeter systems. J. Algebra, 135(1):57-73, 1990.

[15] Anna Felikson, Michael Shapiro, and Pavel Tumarkin. Skew-symmetric cluster algebras of finite mutation type. J. Eur. Math. Soc. (JEMS), 14(4):1135-1180, 2012.

[16] Sergey Fomin, Michael Shapiro, and Dylan Thurston. Cluster algebras and triangulated surfaces. I. Cluster complexes. Acta Math., 201(1):83-146, 2008.

[17] Sergey Fomin and Andrei Zelevinsky. Cluster algebras. II. Finite type classification. Invent. Math., 154(1):63-121, 2003.

[18] Sergey Fomin and Andrei Zelevinsky. $Y$-systems and generalized associahedra. Ann. of Math. (2), 158(3):977-1018, 2003.

[19] Sergey Fomin and Andrei Zelevinsky. Cluster algebras. IV. Coefficients. Compos. Math., 143(1):112-164, 2007.

[20] Mark Gross, Paul Hacking, Sean Keel, and Maxim Kontsevich. Canonical bases for cluster algebras. J. Amer. Math. Soc., 31(2):497-608, 2018.

[21] Robert B. Howlett. Coxeter groups and M-matrices. Bull. London Math. Soc., 14(2):137-141, 1982.

[22] Colin Ingalls, Charles Paquette, and Hugh Thomas. Semi-stable subcategories for Euclidean quivers. Proc. Lond. Math. Soc. (3), 110(4):805-840, 2015. 
[23] V. G. Kac. Infinite root systems, representations of graphs and invariant theory. II. J. Algebra, 78(1):141-162, 1982.

[24] Victor G. Kac. Infinite-dimensional Lie algebras. Cambridge University Press, Cambridge, third edition, 1990.

[25] I. G. Macdonald. Affine root systems and Dedekind's $\eta$-function. Invent. Math., 15:91-143, 1972.

[26] Robert Marsh, Markus Reineke, and Andrei Zelevinsky. Generalized associahedra via quiver representations. Trans. Amer. Math. Soc., 355(10):4171-4186, 2003.

[27] Jon McCammond and Robert Sulway. Artin groups of Euclidean type. Invent. Math., 210(1):231-282, 2017.

[28] Gregg Musiker and James Propp. Combinatorial interpretations for rank-two cluster algebras of affine type. Electron. J. Combin., 14(1):Research Paper 15, 23, 2007.

[29] Nathan Reading. Clusters, Coxeter-sortable elements and noncrossing partitions. Trans. Amer. Math. Soc., 359(12):5931-5958, 2007.

[30] Nathan Reading. Universal geometric cluster algebras. Math. Z., 277(1-2):499-547, 2014.

[31] Nathan Reading and David E. Speyer. Cambrian fans. J. Eur. Math. Soc. (JEMS), 11(2):407447, 2009.

[32] Nathan Reading and David E. Speyer. Sortable elements for quivers with cycles. Electron. J. Combin., 17(1):Research Paper 90, 19, 2010.

[33] Nathan Reading and David E. Speyer. Sortable elements in infinite Coxeter groups. Trans. Amer. Math. Soc., 363(2):699-761, 2011.

[34] Nathan Reading and David E. Speyer. Combinatorial frameworks for cluster algebras. Int. Math. Res. Not. IMRN, (1):109-173, 2016.

[35] Nathan Reading and David E. Speyer. Cambrian frameworks for cluster algebras of affine type. Trans. Amer. Math. Soc., 370(2):1429-1468, 2018.

[36] Nathan Reading and Salvatore Stella. The action of a coxeter element on an affine root system. Preprint (arXiv:1808.05090), 2017.

[37] D. Rupel, S. Stella, and H. Williams. Affine cluster monomials are generalized minors. Preprint (arXiv:1712.09143), 2017.

[38] Dylan Rupel and Salvatore Stella. Some consequences of categorification. Preprint (arXiv:1712.08478), 2017.

[39] Sarah Scherotzke. Component clusters for acyclic quivers. Colloq. Math., 144(2):245-264, 2016.

[40] Ahmet I. Seven. Cluster algebras and semipositive symmetrizable matrices. Trans. Amer. Math. Soc., 363(5):2733-2762, 2011.

[41] Paul Sherman and Andrei Zelevinsky. Positivity and canonical bases in rank 2 cluster algebras of finite and affine types. Mosc. Math. J., 4(4):947-974, 982, 2004.

[42] Salvatore Stella. Polyhedral models for generalized associahedra via Coxeter elements. J. Algebraic Combin., 38(1):121-158, 2013.

[43] Shih-Wei Yang and Andrei Zelevinsky. Cluster algebras of finite type via Coxeter elements and principal minors. Transform. Groups, 13(3-4):855-895, 2008.

[44] Andrei Zelevinsky. Semicanonical basis generators of the cluster algebra of type $A_{1}^{(1)}$. Electron. J. Combin., 14(1):Note 4, 5, 2007.

(N. Reading) Department of Mathematics, North Carolina State University, Raleigh, $\mathrm{NC}$, USA

(S. Stella) Department of Mathematics, University of Leicester, University Road, LEICESTER, LE1 7RH, UK 